\title{
Progetto ARS \\ Archeometria e Remote Sensing per la diagnostica delle Sculture Italiche dall'Abruzzo: risultati preliminari
}

\author{
G. Adinolfi, S. Agostini, V. Belfiore, R. Carmagnola, \\ M.V. Carniel, V. d'Ercole, R. D'Errico, \\ M.G. Di Antonio, E. Di Valerio, M.E. Masci, M. C. Mancini, \\ O. Menozzi, D. Palumbo, I. Zelante
}

\section{Introduction. The sculptures of the Mid Adriatic context: aims and methodologies of the archaeometric analysis and digital imaging}

\author{
Oliva Menozzi ${ }^{1}$
}

The phenomenon of the funerary sculptures is certainly the most remarkable expression of the archaic culture in the mid Adriatic context and it includes several funerary sculptures made of local limestone, dating between the $6^{\text {th }}$ and the $5^{\text {th }}$ centuries BC..$^{2}$. However, it must be clear that the use of monumental tombstones in form of simple baetyli, that is rough menhir used as semata (markers) of the most monumental tombs, is already attested in the cemetery of Scurcola Marsicana since the $7^{\text {th }}$ century $B C^{3}$. These funerary sculptures seem to be typical of the 'Mid-Adriatic' area, intending with this term the area of the modern regions known as Abruzzo, southern Marche and Molise, which were part of the 'paleo-Sabellian' cultural koine between the $6^{\text {th }}$ and the $5^{\text {th }}$ centuries BC. They were representing important local figures or chiefs, attesting a socially differentiated society of Archaic Abruzzo (Fig. 1); and they were not used only for a phenomenon of 'heroization', which is widely attested in Archaic Mediterranean contexts, especially in association with tumuli, but also for remarking role and status of the dead person. Another interesting and important element for these sculptures is the presence of inscriptions, which are among the most representative linguistic samples of this cultural context. ${ }^{4}$

These sculptures can be organised into three different groups: the first two including stelai with sculptures in relief, i.e. tombstones bearing in relief part of the figure or the complete figure, and the third proper 'statues'. ${ }^{5}$ The most ancient example of the first group comes from Guardiagrele (A in Fig. 1): it consists of an anthropomorphic stele with the face realized in a very low relief and wearing two orientalizing kardiophylakes, which are incised on the breast and on the back, joined with a belt with geometric

\footnotetext{
${ }^{1}$ Director of the CAAM (Centro di Ateneo di Archeometria e Micronalisi), Università G. d'Annunzio of Chieti-Pescara.

${ }^{2}$ Examples of these tombstones have been found at Rapino, Guardiagrele, Collelongo, Loreto Aprutino, Capestrano, Monte Pallano, Bellante, Manoppello. CianfarAni 1976, pp.31-32; CiANFARANI, FrANCHI Dell'OrTo, LA Regina 1978, pp.115-120; D’ERCOLE, PAPI, Grossi 1990, pp.201-206; COlONNA 1999, pp.104-109.

${ }^{3}$ D’ERCole, PAPI, Grossi 1990, p.201; D’ERCOLE 1991, pp.252-270.

${ }^{4}$ For the debated question among the scholars of the use of conventional names as 'Medio-Adriatico', 'Sud-Piceno' or 'Paleosabellico': cfr.Prosdocimi 1977, pp. 29-74; 1878; 1985, pp.35-56; 1987, pp. 113; DURANTE 1978, pp. 789-824; MARINETTI 1984 and 1985.

${ }^{5}$ COLONNA 1992, 99-100.
} 
decorations, and a necklace with pendants. On the sculptures from Penna Sant'Andrea ${ }^{6}$ (B in Fig. 1), dating to the $5^{\text {th }}$ century BC, only the faces were represented, as probably on a fragment from Loreto Aprutino ${ }^{7}(\mathrm{C}$ in Fig. 1): it seems to be conceivable that they wore a hat or helmet, as the socket at the top of the head seems to suggest. Only the faces and some ornaments or military elements are represented in this first group, as 'metonymic' representation of the dead person, through his face, and of his social status and role, through the representation of the few ornaments and hat/helmet. In spite of the very simple and rough representation of the faces, the stelai from Penna Sant'Andrea (one complete and two fragmentary) represent also the longest texts of the Archaic culture in this context.

The second group could be seen as an iconographic evolution of the first one, showing, again in relief, the complete figure, as we can see in one of the two tombstone from Bellante $^{8}$ (E in Fig. 1), which presents the whole figure in low relief; the stele is smaller than the others and the representation is quite rough and simple, but, again, with a quite long text, in this case arranged around the figure. The attitude of the figure, with both arms folded on the bust, seems to evocate the funerary gestures expressed also in the two statues from Capestrano (see below), in the bust from Atessa/Monte Pallano and probably also in the fragmentary statue from Rapino (just a fragment of the lower part of the statue is preserved, relating the pelvis, so in case the statue would have been represented with the arms along both sides, there would have been at least remains of them on both sides of the hips).

Also a fragment from Collelongo ${ }^{9}$ is generally included within the second group, although Colonna is indicating the appurtenance to the third one ${ }^{10}$ because it can be considered almost a statue: including the legs of a male figure from the knees to the feet (D in Fig. 1). This sculpture is known as 'Gambe del Diavolo' ('Devil's legs'), and, because of its very high relief and larger size, certainly seems to anticipate or evocate the statues: it is, in fact, more detailed in its representation and larger, almost at human size.

The third group consists of tridimensional standing statues, more complete from the sculptural point of view, but also in their military attributes and personal ornaments ${ }^{11}$ (Fig. 2). A head from Manoppello (Fig. 2A), which can be included in this third group, has small, circular eyes, deeply incised, small ears and wears a torques, which finds

\footnotetext{
${ }^{6}$ Which have been found in the area of the Sanctuary of Montegiove (municipality of Cermignano), in a funerary context not far from the sanctuary, where circular tumuli are still visible from remote sensing of the area. The three tombstones (one complete and the other two preserved just in fragment and possibly belonging to a single second stele) are now preserved in Chieti, Museo Archeologico Nazionale d'Abruzzo, Villa Frigerj (from now on MANdA, Villa Frigerj).

${ }^{7}$ In the modern province of Pescara and now preserved in Chieti, MANdA, Villa Frigerj.

${ }^{8}$ In the province of Teramo, but now at the Archaeological Museum of Naples (MANN).

${ }^{9}$ In the province of L'Aquila, in the Marsican context. Now it is preserved in Chieti, MANdA.

${ }^{10}$ ColonNA 1992, pp. 92-127.

${ }^{11}$ Found at Manoppello (Pescara province), Rapino (province of Chieti, not far from Guardiagrele), two at Monte Pallano (Tornareccio-Atessa, in the southern part of Chieti province), including the so-called 'Torso di Atessa' and the fragment from Acquachiara, and two (preserved) at Capestrano (but other fragmentary statues found in the area have been lost), including the Capestrano Warrior and a fragment of female bust. Apart from the former, which is preserved in Chieti, Museo Archeologico Nazionale La Civitella (from now on MAN La Civitella), the rest of the statues are now preserved in the Archaeological Museum of Chieti.
} 
parallels in numerous iron examples from 'south-picene' necropolis ${ }^{12}$, dating to the second half of the $6^{\text {th }}$ century BC. In addition, the socket on the head seems to suggest again a hat or helmet covering the head attesting the social and/or military role of the represented person, and the kind of representation of the face, although very damaged, seems to suggest the rigid prospect of a mask, with funerary or military implications ${ }^{13}$.

The statues from Atessa/Monte Pallano, Rapino and the female bust from Capestrano
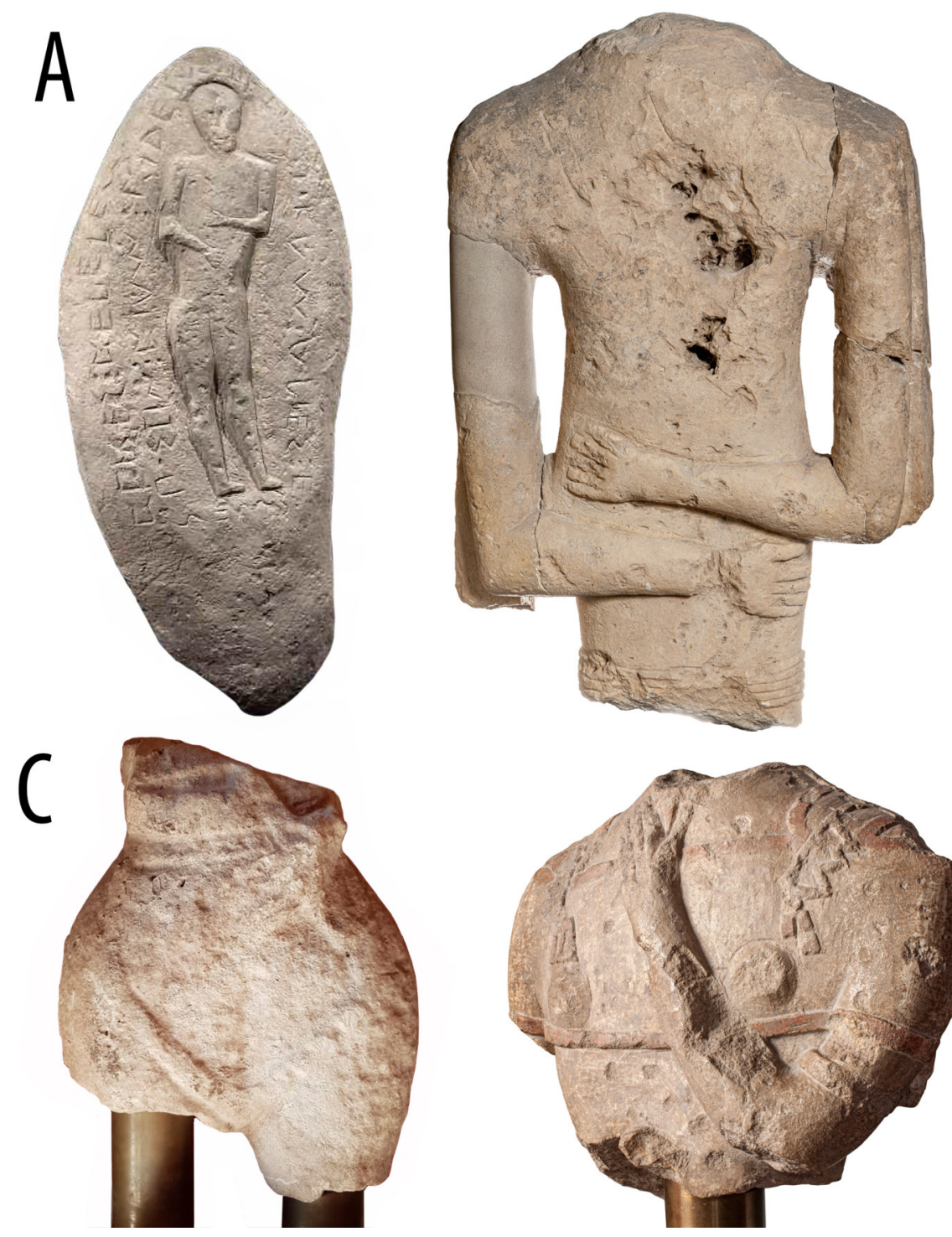

B

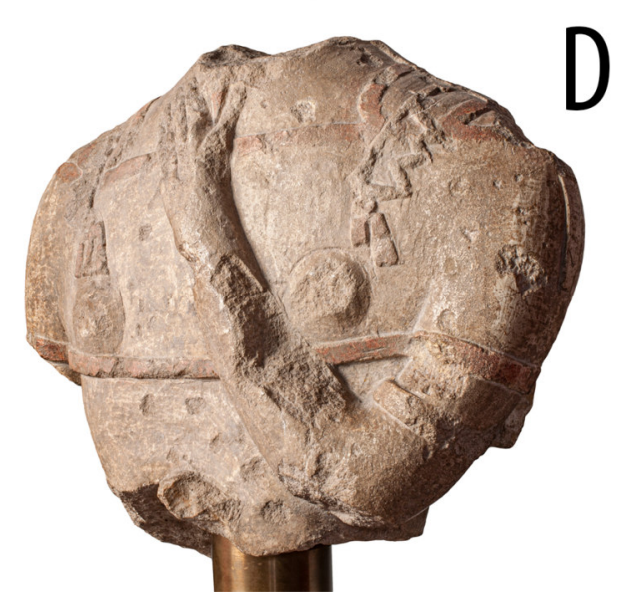

Fig. 1 A-Stele from Bellante; B-Torso from Atessa;/Pallano C-Fragment of statue from Rapino; D- the female fragment of statue from Capestrano.

\footnotetext{
${ }^{12}$ BRIZIO 1902, pp.229-257.

${ }^{13}$ The use of funerary masks is quite well attested in Etruria (for details see below the contribution of V. Belfiore), Archaic Rome, as well as in northern Greek, Macedonian or Illyrian contexts, as the case of Archontiko (not far from Pella), where a funerary golden mask, together with jewels in gold, have been found in the grave of a priestess (g.n. 458), dating to the $540 \mathrm{BC}$; the mask, for male burials in this context, can be associated also to military equipment, as in the case of the golden masks which have been found at Sindos and dating around the 520 BC. DESPINI, SCHÜRMANN AND GISLER 2009, pp. 20-65
} 
(B,C,D in Fig.2), are fragmentary remains of free-standing statues bearing personal ornaments, such as belts, fibulae and pendants, as well as weapons, as a knife in the example from Rapino, which can be dated as the earliest of the third group, specifically for the use of the short iron knife.
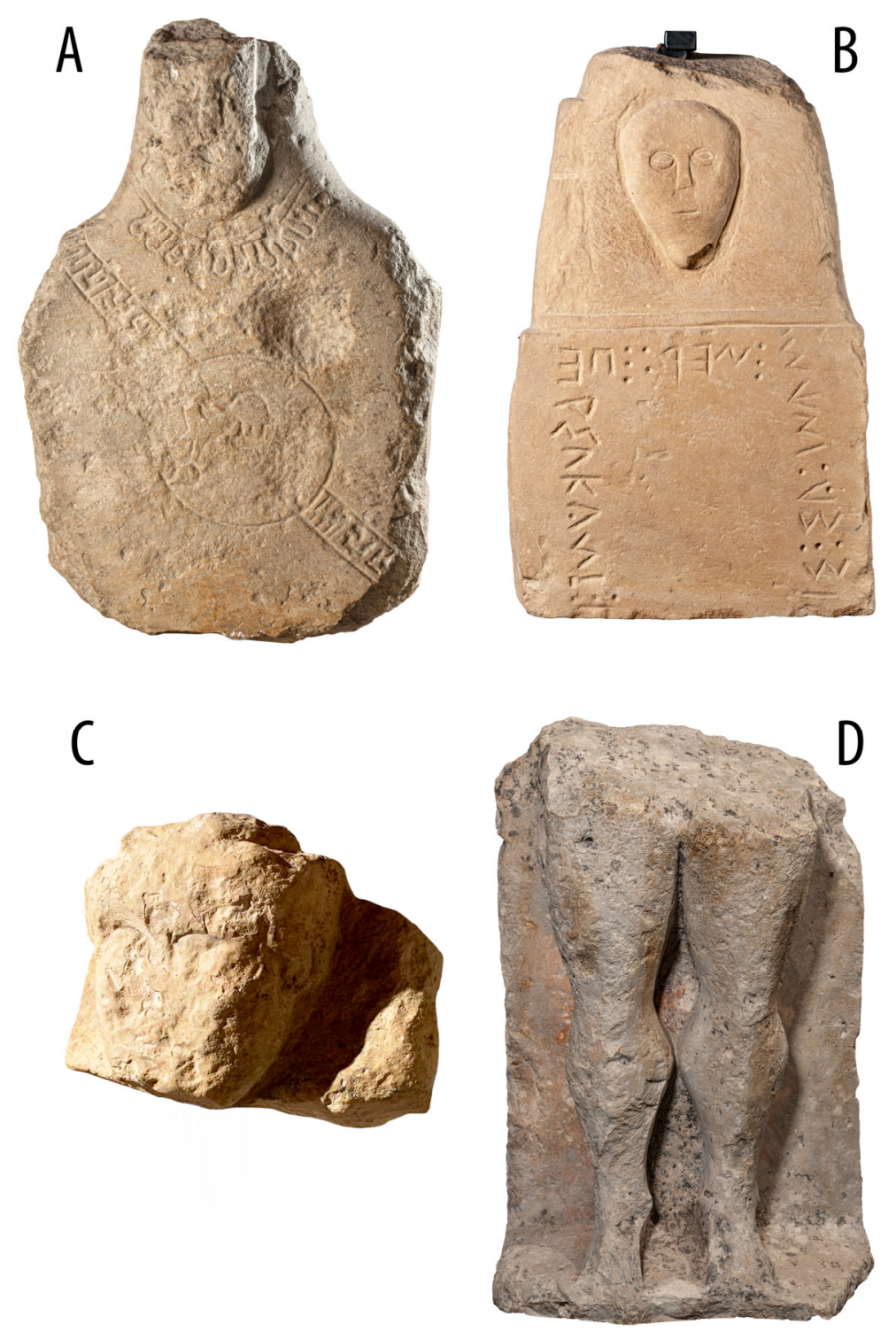

Fig. 2 A- The Stele from Guardiagrele; B- one of the Stele from Penna Sant'Andrea; C- the fragment of the stele from Loreto Aprutino; D- the so called 'Gambe del Diavolo' from Collelongo.

The female bust from Capestrano (D in Fig. 2), although very fragmentary, is particularly important because it represents the only known example in the Italic world of a female statue, with the easy implication of an important female role in the 'PaleoSabellic' or 'Safin' culture. It is certainly an attractive interpretation, and it can also be conceivably confirmed by the comparison with the richness of some female tombs dating to this period found at Campovalano, Loreto, Fossa, Capestrano and so on, attesting the existence of 'local princesses'. However, it is difficult to interpret their 
role, either they belonged simply to an important clan, or to leading figures in the local political asset (but the statue is representing a young, probably teenage, woman and is therefore quite unconceivable that she could have reached a leading position).

A particularly good example of standing statue is the famous 'Capestrano Warrior' (Fig. 3), which is the best preserved of these sculptures and is also presenting the most
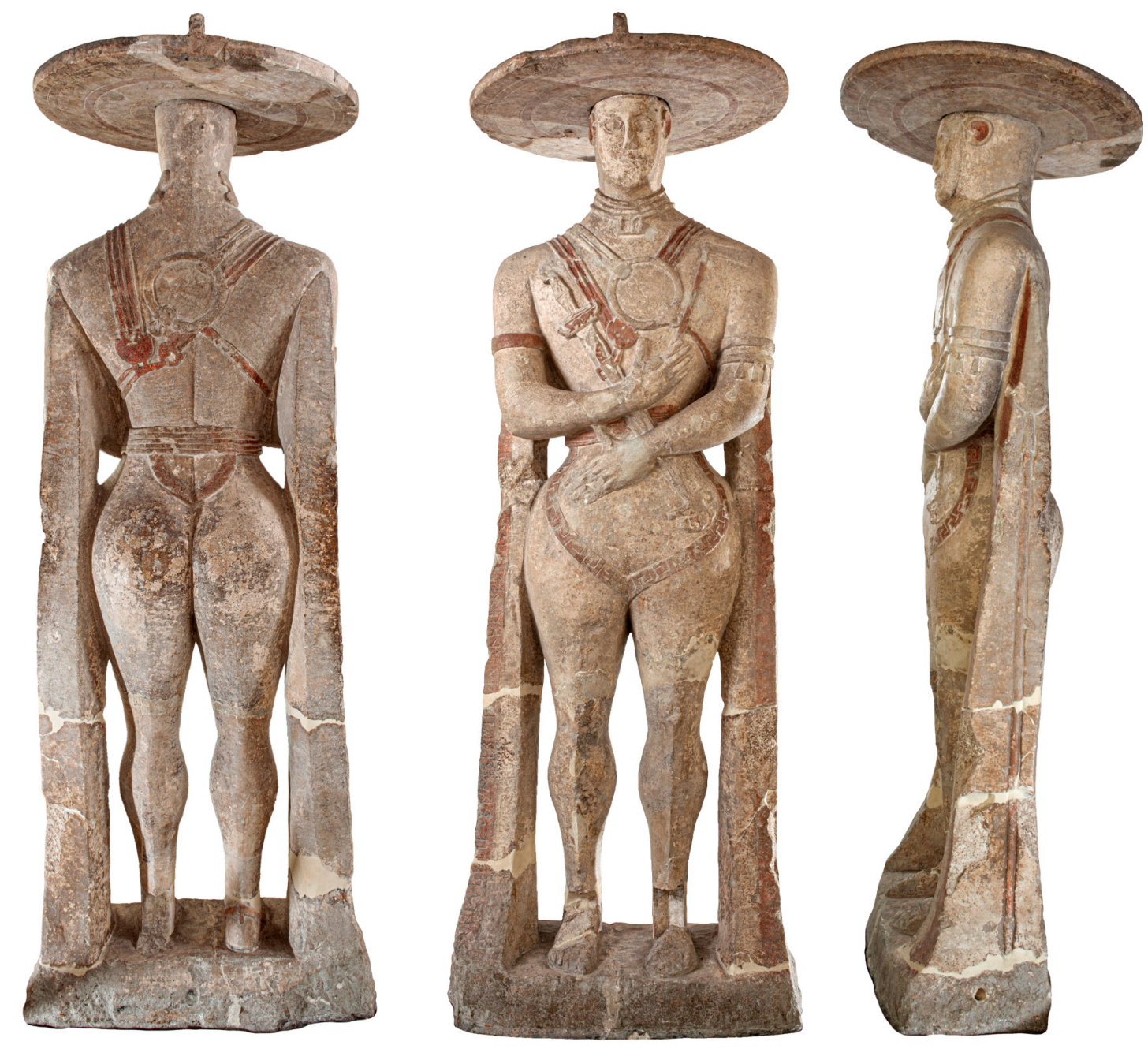

Fig. 3 The Statue of the Warrior from Capestrano.

complete set of ornament and weapons: representing a sort of exemplificative panoplia for the $6^{\text {th }}$ cent. $\mathrm{BC}$, because the kind of sword which is used in the region not before the second quarter of the $6^{\text {th }}$ century $\mathrm{BC}^{14}$. It stands on a rectangular base between two pillars with the representation of two spears incised on the outer side of each pillar. $\mathrm{He}$ wears a short skirt/apron fastened with a belt, on the bust two kardiophylakes, a sword in its sheath, an axe and a very particular helmet or hat, with a very wide brim and halfspherical headgear probably decorated on the upper part. The military attributes indicate

${ }^{14}$ D’ERCOLE 2015. 


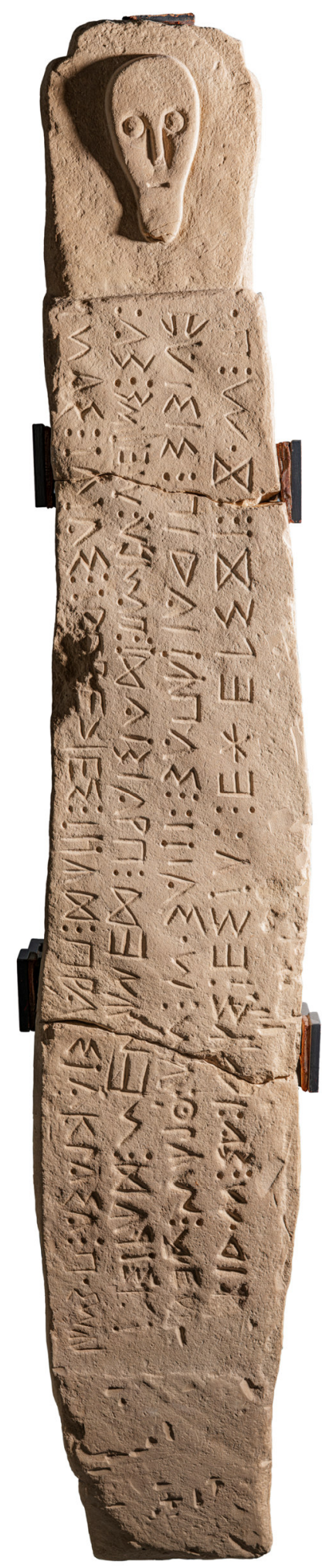

Fig. 4 Inscriptions from Penna S.Andrea. a leader, a king if the word raki of the inscription carved on one pillar is correctly interpreted as equivalent of the Latin rex. The mention of a 'monarchic' role is extremely interesting in this context, where there are no other sources concerning the political and historical evolution of these tribes. However, if the leading role is clear, both from the inscription and from the kind of representation of the statue, especially for its panoplia and attributes, it is difficult to define properly the real meaning of this role and how wide could have been, if limited within a clan or tribe or wider. Moreover, if the inscription has been wider already debated and published ${ }^{15}$, both concerning the term 'raki' and the name of the person, Nevius Pompuledius, it is also particularly interesting and new in this context to emphasize the mention of an 'official sculptor' signing the statue, Aninius, attesting its role, not far from what is happening for instance in Etruria, but also in Greece, Magna Graecia and Sicily, where 'speaking statues' are often mentioning the donor of the statue, or the name of a specific deity, but, sometimes, also the name of the sculptor ${ }^{16}$.

It is not so rare, as mentioned above, to find 'paleo-sabellic' inscriptions on these sculptures, including the examples from Penna Sant'Andrea and Bellante just mentioned, and in the former case the date of the inscriptions is in apparent contrast with the 'evolution' of the types. The inscription on the pillar of the Capestrano Warrior (Fig. 5) is dated to the mid $6^{\text {th }}$ century $\mathrm{BC}$ and the inscriptions from Penna S.Andrea to the $5^{\text {th }}$ century BC (Fig. 4), but their sculptural features seem to suggest that the former is the 'more developed' and the latter the 'more primitive' types. However, the iconographic evolution of the types must be dated before the introduction

\footnotetext{
${ }^{15}$ See among the latest studies CALDERINI, NERI, RUGGERI 2007; LA REGINA 2010, pp. 230-273; CRAWFORD 2011, 226-227, Vestini/ Avfinum 1.

${ }^{16}$ Particlarly famous is the case at Delos of an Archaic base (inv. Delos A728) mentioning Euthykartidas as donor and sculptor of the statue: Boardman 1978, fig.56.
} 


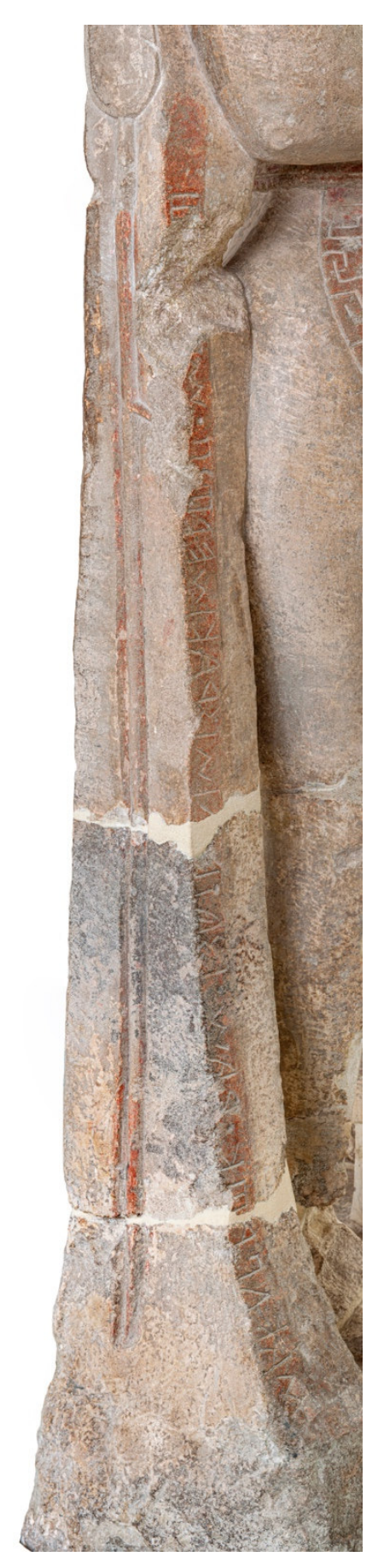

Fig. 5 The inscription on the pillar of the Capestrano Warrior of writing ${ }^{17}$; and the different groups continued to be used for long and probably the three different types coexisted in the local tradition and attest the different tastes of customers and artists, or of tribes and areas. These sculptures are the expression of a local artistic production, which in its simplicity shows strong connections with Etruscan and Greek art ${ }^{18}$ although maintaining its originality as well as differentiated message. For example, the orientalizing decorations of ornaments and kardiophylakes on the sculpture from Guardiagrele and on the sword sheath of the Capestrano Warrior show strong influences from the Etruscan and Faliscan area, while the geometric patterns on the belts of the fragments from Pallano and Rapino, as well as from the Capestrano Warrior, are clearly influenced by the Greek world. The folded position of the arms of the sculptures from Capestrano, Bellante and Pallano/Atessa and probably Rapino, as mentioned above, is widely attested in Greek, Etruscan and Daunian funerary sculptures, reminding to funerary meanings; moreover the position of one of the arms on the abdomen and the other on the breast, which can be found in the bust from Pallano/Atessa, in the Warrior and in the female bust from Capestrano, indicating particular symbols of their status, i.e. the axe in the warrior and the bulla in the female bust, is typical of orientalizing and archaic Etruria. The use of the axe as a symbolic element of a strong leading role is quite attested in Archaic Greece, as the axe used as an attribute in the representation of the god Dike in the Archaic period represented while fighting against Adikia and brandishing against her a labrys or pelekis ${ }^{19}$, as symbol of regality as well as ritual attribute; moreover, in mythology, an axe was the symbol of the power of the queen of the Amazons, which was then taken by Herakles and he then gave it to Omphale, queen of Lidia, specifically to emphasize her regality ${ }^{20}$.

\footnotetext{
${ }^{17}$ Colonna 1992, pp.99-100.

${ }^{18}$ COLONNA 1992, pp.92-127.

${ }^{19}$ Attested in a black figure metopal representation on an Attic amphorain the Kunsthistorisches Museum (Inv. n. 3722, Beazley 200050) at Vienna as well as in one of the metopal scenes of the Cypselus chest as described by Pausanias (V 17, 5-19, 10.), cfr. MENOZZI 2019, in part. pp 303-305.

${ }^{20}$ MENOZZI 2019, in part. p 304.
} 
However, the models are not allochthonous and the external influences, in these original expressions of the Italic art, are deeply re-elaborated in a local tradition, and they can even differ from statue to statue, showing also a quite interesting internal cultural complexity.

The interpretation of these sculptures has always seen them as semata used singularly as markers of important tombs; however, if undoubted is their use as indicators of a social role and status, the use as just single markers has been then totally reviewed. The Capestrano Warrior itself has been found together with fragments of other one or two male statues ${ }^{21}$, and also with the female one, attesting probably a 'familiar group of statue', intending not in term of 'sculptural group' but of single statues grouped in the same funerary context because related to the same aristocratic clan, a local leading genos. This is an interesting use of a 'familiar group' which is probably earlier attested locally also by the groups of betyli or menhir which have been found at Scurcola Marsicana, Fossa, Celano, Bazzano ${ }^{22}$. This use is also known in Greek funerary contexts $^{23}$ as for instance the Archaic statues made by Aristion of Paros (who was signing the statues) representing the kore Phrasikleia (her name is indicted in the inscription) and a kouros found with her (both dating around the $550 \mathrm{BC})^{24}$, which were used as markers of a tomb and were then soon de-functionalized and buried together in antiquity probably for a phenomenon of damnatio memoriae because members of the important aristocratic genos of the Alcmeonides, who were banned by Pisistratus around the $538 \mathrm{BC}$.

Similarly, in Etruscan contexts, as at Casale Marittimo (Volterra), Vetulonia and Gozzo Veronese the use of funerary statues representing groups is also attested ${ }^{25}$ and in some cases, as the latter, including both female and male statues.

The Italic sculptures have often been published without their original contexts, determining not only some misunderstandings in their use, dating and role, but also a quite 'antiquarian' approach in the analysis which has for long been specifically based just on artistic features. Only recently the stelai and statues have been compared with tombs and funerary equipment from close necropolis, both in chronological and topographic terms. This passage is fundamental to investigate more closely also the social contextualization with a more anthropological approach.

Moreover, the kinds of stone which have been used to realize the sculptures, as well as their sources and quarries have been investigated just recently, and in the past they were seen just as 'realised in limestone', while the different physical features of the stones are important to understand some of the specific differences generally seen as 'artistic meanings'. The study of the typologies of stones, of their physical features, in combination with the tool marks on the surfaces, could give important information

\footnotetext{
${ }^{21}$ CELLA 2012; cfr also the contribution of V. d'Ercole below, describing in detail the other fragments and the possible reconstruction of this 'group'.

${ }^{22}$ See the contribution of V. d'Ercole below.

${ }^{23}$ Quite suggestive is also the parallel proposed by Alessandro Naso during the a recent conference (in Chieti 'Scultura Preromana', 28-29 November 2019) with the famous 'Group of Geneleos' from the Heraion at Samos, in this case the group is representing the members of a family of donors, but found in a sanctuary and not in funerary context. Similarly also the case of the 'Group of seated statues' from the Apollonion of Dydime (Miletus) can be mentioned as example of 'familiar group' in the archaic period, although again, in a religious context.

${ }^{24}$ BOARDMAN 1978, pp.73-74, fig. $108^{\circ}$.

${ }^{25}$ See MAGgiani 1999; Esposito, MAGGiAni 2006. Some discussion about these cases are presented in the contributions of Vincenzo d'Ercole, just below in this paper.
} 
about the kinds of tools and techniques which have been used for the realization of these stelai and statue. Similarly the colors, which are quite evident in some of them, have never received specific attention, apart interpreting them as indicators of more or less artistic currents.

Therefore, in order to get more information about these important examples of Italic finds, a large team has been involved in the project $\mathrm{ARS}^{26}$, with the aim of a wider approach and a multi- as well as inter-disciplinary view. Data and results are constantly shared among the numerous scholars and specialists involved, as also protocols, results and typologies of analysis are widely discussed in advance within the team for a truly shared approach.

The methodologies and technologies involved are quite numerous. The HD photography is a starting point for the photographic and photogrammetric documentation of the sculptures, for the stereoscopic and 3D modeling, as well as for the digital imaging and photo-interpretation. Every step is obviously aiming the analysis, the documentation and the dissemination of the results, not only for scientific purposes, but finalized to a possible fruition of the data also by the 'wider public'.

The remote sensing is based on different methodologies, from a basic photointerpretation, to the use of filters based on different colors, as well as of UV fluorescence photography and spectroscopy, and of B/ $\mathrm{N}$ and false color Infrared range, using, in this way, both visible and invisible spectrum.

Concerning the study of the litho-types, for obvious reasons, a non-invasive approach has been chosen, avoiding therefore thin sections and destructive SEM analysis for the moment; therefore the petrographic analysis are made through a digital microscope and the results are then combined also with other techniques as the use of ultrasounds, reflectance, portable diffractometry and fluorescence.

The macro- and micro-mapping of the tool marks are based on both on a direct analysis, on the use of the digital microscope, as well as on the analysis of the complete set of images, overlapping them and drawing the different typologies of marks, in order to understand the main tools which have been used, but also the way and the intensity of their use. This mapping is also giving interesting results for recognizing modern interventions (as old restorations or false marks and integrations). Thanks to the collaboration with restorers, also an analysis of the main restorations is in progress, because these sculptures are mainly coming from finds dating to the beginning of last century, when they were polished and restored before the exhibition in the museums, but without any report of which kind of work was done for each of them.

Moreover, the use of the infra-red thermo-camera and the analysis of the data of the reflectance from the laser-scanner ${ }^{27}$, can give interesting data about the substratum of the colors, the conditions of the sculptures, as well as the changes of the consistence of the substratum, which is often indication of later restorations of false elements.

The re-contextualization of these finds is another important goal, both from the cultural and topographical point of view. Is now possible to associate some of the sculptures to necropolis and specific tombs, as well as to compare the ornaments and weapons represented, with the funerary equipment, which are now numerous because

\footnotetext{
${ }^{26}$ The project ARS is born within the European project EuroTeCH (European technologies for Cultural Heritage at risk) and specifically thanks to the collaboration of two partners of the project: CAAM (Centro di Ateneo di Archeometria e Microanalisi) of the University G. d'Annunzio of Chieti-Pescara) and Pegaso srl (Rome), with the MANdA Villa Frigerij and the MAN La Civitella, both in Chieti.

${ }^{27}$ MenOZZI, TAMBURRino 2012; Meschini, PElliccio 2013.
} 


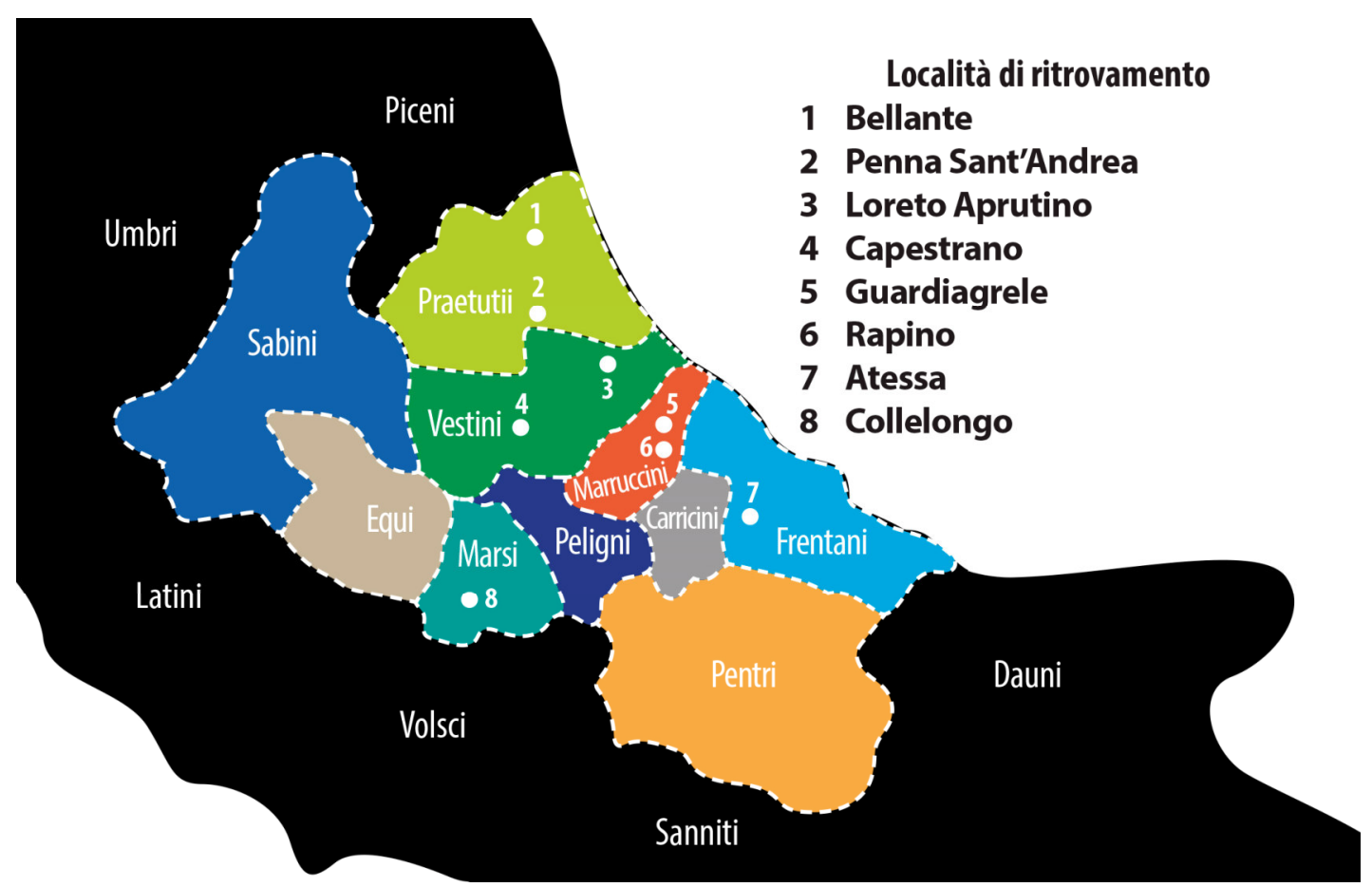

Fig. 6 Map with the locations of the sculptures on a base with the indication of the main mid. Adriatic tribes. It must be emphasized here that themap is indicating the situation of the tribes as attested by later sources not before the $4^{\text {th }}$ century $\mathrm{BC}$, while the sculptures can be dated between the late $7^{\text {th }}$ and the $5^{\text {th }}$ century BC.

Mappa con la localizzazione dei rinvenienti di sculture italiche citate nel testo su base geografica indicante i territori delle principali tribù medio-Adriatiche. Bisogna però segnalare che la situazione territoriale delle tribù è quella testimoniata dalle fonti più tarde, a partire dal IV secolo a.C., mentre i rinvenimenti scultorei datano tra VII e V sec.a.C.

more widely excavated than before: this is an important step for defining a closer dating and for a more contextualized view.

The results, apart for papers which can be seen as interim reports, will be definitively published in a volume which will present for each sculpture a form filled with the several results organised together in close combination and correlation; in this way it will be easier to give a more homogeneous view instead of separate papers on single researches. A further use of the results coming from the analysis is the possibility of a wider and more inclusive valorization, using the $3 \mathrm{D}$ models of the sculptures as 3D bases for interactive forms illustrating to the wide public results, features, marker tools and data coming from this project and then published both for 'scientific purposes' but also for a more touristic perspective. 


\title{
Le metodologie, il team risultati preliminari del digital imaging
}

\author{
Gloria Adinolfi, Silvano Agostini, Valentina Belfiore, Rodolfo Carmagnola, \\ Maria Violeta Carniel, Vincenzo d'Ercole, Rocco D'Errico, \\ Maria Giorgia Di Antonio, Eugenio Di Valerio, Maria Emilia Masci, Maria Cristina \\ Mancini, Oliva Menozzi, Donato Palumbo, Ilaria Zelante ${ }^{28}$
}

L'alto numero di studiosi e specialisti che stanno lavorando nel team è dato dall'approccio differenziato che deve essere necessariamente multidisciplinare ma anche interdisciplinare. Nella maggior parte delle analisi infatti non si prevede una edizione finale dei risultati con dati forniti separatamente, ma con una gestione condivisa dei protocolli, dei risultati e delle analisi per una interpretazione più completa e interdisciplinare del dato; nella redazione finale di un volume monografico, infine, si intende integrare tutti i dati e i risultati in schede specifiche su ogni singola scultura che permettano una ricostruzione integrata della storia del manufatto. D'altronde gli stessi protocolli sono continuamente testati dai diversi membri del team con punti di vista e approcci scientifici molto differenziati. Inoltre i dati vengono rigorosamente incrociati e discussi perché vi siano riscontri continui che possano suffragare ipotesi e suggestioni. L'uso della fotografia rappresenta la base di partenza ed ha un'ampia serie di applicazioni in questo progetto (cfr. infra il contributo di R. D'Errico). Si parte dalla fotografia ad alta risoluzione, che serve a far risaltare $i$ dettagli, fino alla fotogrammetria, alla stereoscopia, al 3D modeling sia a fini analitici sia divulgativi. È chiaro che tutte le analisi siano finalizzate allo studio delle sculture e alla diagnostica dei reperti, anche al fine della conservazione, ma ci siamo prefissi, come ulteriore finalità di questo progetto, anche una divulgazione agile dei risultati, più diretta ed esplicativa, perché anche il grande pubblico possa fruire dei risultati delle analisi, anche attraverso modelli 3D che fungano da schede tridimensionali su cui i dati vengano resi accessibili in modo interattivo (fig.7).

Il remote sensing delle sculture è declinato in base a diverse metodologie e tecniche. Si opera sia un remote sensing di tipo foto-interpretativo indiretto e passivo, attraverso l'utilizzo di filtri e di colori dello spettro del visibile, ma si stanno utilizzando anche tecniche legate ad una fluorescenza dell'ultravioletto $o$ allo spettro dell'infrarosso, con tecniche di tipo più diretto, con l'esaltazione dello spettro del non visibile. Quindi infrarosso B/N, infrarosso a falso colore, ultravioletto per fluorescenza, sono tra le tecniche che abbiamo già applicato sulle statue ai Musei di Chieti e che intenderemmo anche utilizzare sulle sculture al Museo di Napoli, come le stele di Bellante e di Crecchio. Tra le varie tecniche si sta anche utilizzando il protocollo che $\mathrm{i}$ colleghi della Pegaso srl stanno da anni utilizzando con il CNR sulle tombe dipinte di Tarquinia, sulle metope del Sele e di altri contesti (cfr. infra il contributo di Gloria Adinolfi e Rodolfo Carmagnola). In questo caso i protocolli sono in fase di adattamento alla scultura, che presenta problematiche diverse da quelle della pittura.

Per l'analisi dei litotipi (cfr. infra il contributo di Silvano Agostini), si è deciso di intervenire con protocolli analitici assolutamente non invasivi, basati sull'indagine petrografica attraverso microscopia digitale e sui dati provenienti da analisi più tecniche, quali ultrasuoni, riflettanza, nonché da diffrattometria e fluorescenza portatili.

\footnotetext{
${ }^{28}$ Ruolo e istituzioni di appartenenza di tutti gli studiosi sono indicati nei singoli contributi.
} 
Particolare attenzione è riservata alla mappatura delle micro- e macro- tracce di lavorazione per una sistematica ricostruzione degli attrezzi utilizzati (cfr. infra il contributo di Eugenio Di Valerio), sia attraverso un'analisi autoptica diretta, grazie anche all'utilizzo della microscopia digitale, sia dallo studio delle immagini ad alta definizione e da spettro del non visibile. È fondamentale riuscire a mappare e catalogare ogni singola traccia di lavorazione, soprattutto per verificare quali fossero i segni di

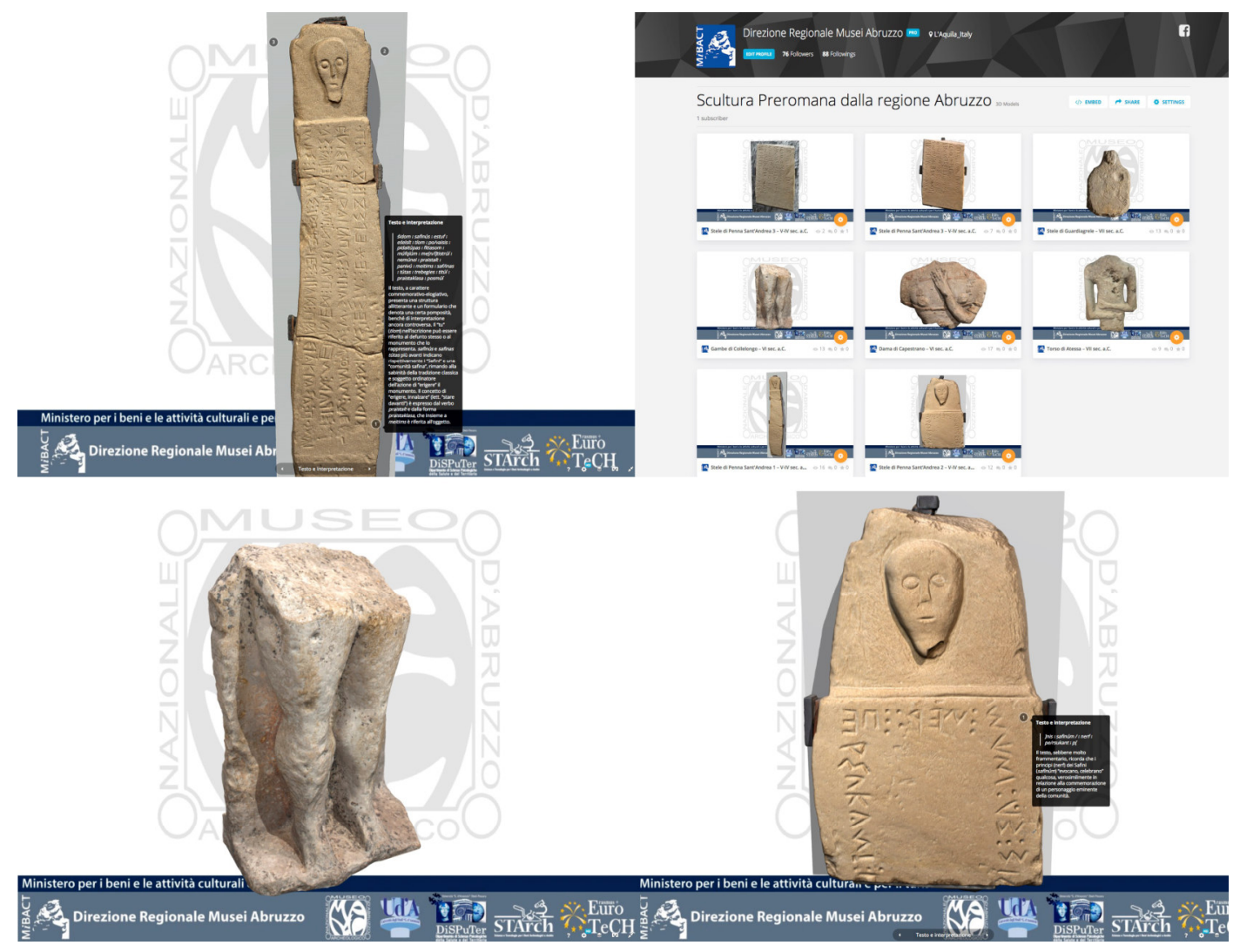

Fig. 7 I modelli 3D interattivi pubblicati sulla pagina dedicata alle sculture preromane sulla piattaforma Sketchfab della Direzione Regionale Musei Abruzzo.

lavorazione ascrivibili in antico, e quali, invece, possano essere attribuibili ad eventuali ritocchi, restauri o addirittura aggiunte moderne.

È chiaro che alle analisi dirette che si stanno ora eseguendo, vengono associati dei protocolli archeometrici di tipo non invasivo, che possano essere di supporto ai risultati. Tra le analisi non invasive, quella che si sta pianificando al momento è l'utilizzo di fluorescenza e diffrattomeria portatili, che possano supportare con analisi chimico-quantitative i dati della microscopia digitale, del remote sensing e delle analisi autoptiche. Molto utile, per capire lo stato di conservazione di ciascun reperto, è anche una diagnostica di dettaglio, sia di tipo diretto, che attraverso l'utilizzo di Telecamera ad infrarosso termico e analisi degli spettri di riflettanza del laser scanner.

Nell'analisi delle sculture si sono notati i resti di numerosi interventi di consolidamento che devono essere avvenuti durante le prime fasi di musealizzazione delle sculture e di cui non si hanno notizie. A tal fine abbiamo coinvolto anche tecnici restauratori, che stanno eseguendo una mappatura di dettaglio di tali piccoli e grandi restauri che hanno comunque condizionato la storia delle sculture.

La stessa ri-contestualizzazione dei reperti sembrerebbe ormai scontata, ma si stanno pian piano scoprendo piccole novità e interessanti vicende relative a contesti, 
aree, necropoli e attori dei rinvenimenti che sarà utile approfondire e pubblicare insieme ai reperti perché vengano tramandate quelle che sembrano essere ricerche, non solo sul territorio ma anche nei vari ambiti sociali e culturali agli albori dell'archeologia preventiva e della tutela del patrimonio culturale. A tal fine si sta operando una ricontestualizzazione culturale e topografica ove possibile accanto ad un lavoro di ricerca d'archivio.

Di recente si è provveduto alla scansione con laser scanner di tutte le statue sia per ottenere nuvole di punti di dettaglio da sovrapporre alle immagini ad alta definizione, ma anche e soprattutto per operare filtraggi e analisi degli spettri da riflettanza, ${ }^{29}$ che sono molto interessanti dopo il merging con la nuvola di punti da scansione laser (cfr. infra il contributo di Donato Palumbo).

Gli esiti attesi del progetto sono quindi molto differenziati, dalla schedatura alla pubblicazione scientifica, da modelli 3D per motivi analitici a quelli per una fruizione museale anche da remoto, dalla ri-contestualizzazione culturale delle sculture a possibili virtual tour che possano arrivare anche ad un pubblico di non addetti e magari incuriosirlo e spingerlo a visitare i musei che le ospitano.

Il progetto è ancora alle sue fasi iniziali, ma ha già prodotto i primi incoraggianti risultati che sono stati anche presentati al Convegno 'La Scultura Preromana', tenutosi a Chieti il 28 e 29 novembre 2019. Gli esiti sono ancora del tutto preliminari e alcuni dei test sono da considerarsi in fieri, ma val la pena di presentare almeno una sintetica selezione delle novità interessanti e di alcune 'sfiziose curiosità'.

La statua del Guerriero di Capestrano risulta senz'altro la più nota di questa produzione artistica e culturale, e in quanto tale ritenevamo fosse anche la più investigata. I protocolli analitici sulla statua sono stati dunque eseguiti per omogeneità nella documentazione, tuttavia è stato subito chiaro che questo attore principale del panorama scultoreo d'Abruzzo continua a riservare sorprese e novità.

Dalla documentazione fotografica all'ultravioletto, che sfrutta il range del near $U V$, è stato possibile innanzi tutto confermare la presenza, già da alcuni ipotizzata, di una maschera bianca sul volto del guerriero, che risulta ben evidente dall'esposizione agli ultravioletti che solitamente esalta soprattutto i bianchi in particolare il carbonato basico di piombo ${ }^{30}$ : il volto rigido e poco particolareggiato del guerriero è dunque in realtà ascrivibile ad una maschera resa da uno strato pittorico bianco (Fig. 8). Non è questa la sede per approfondire l'uso di maschere in ambito funerario, ben note nel mondo romano arcaico ed etrusco, o in ambito militare, come potrebbe suggerire l'esemplare dal Molise, purtroppo decontestualizzato, ma certo la conferma della presenza di una maschera di questo tipo sul Guerriero di Capestrano è un dato importante per dirimere anni di dibattiti, di ipotesi e smentite (cfr. infra il contributo di Valentina Belfiore). Dalle immagini risulta evidente come la maschera si fermi sulla fronte e non presenti continuità con il copricapo e segua i bordi laterali del volto, mentre l'incarnato è eseguito con minor quantità di bianco. Prossimo step è ora l'analisi chimica dei pigmenti o colori utilizzati, attraverso però indagini non invasive, quali diffrattometria e/o fluorescenza portatili, che possono esser effettuate in situ e non comportano una campionatura invasiva.

Le immagini ad alta definizione e quelle ad infrarosso $\mathrm{B} / \mathrm{N}$ della panoplia del guerriero hanno permesso di documentare diverse particolarità sino ad ora

\footnotetext{
${ }^{29}$ MenOZZI, TAmburrino 2012; Meschini, Pelliccio 2013.

${ }^{30} 2 \mathrm{PbCO}_{3} \cdot \mathrm{Pb}(\mathrm{OH})_{2}$.
} 
assolutamente mai rilevate. Una analisi di dettaglio verrà fornita in una pubblicazione più ampia dei risultati, ma val la pena di segnalare, in questa sede, che uno dei due kardiophylakes che il guerriero indossa, e nello specifico quello del retro, è decorato al centro con il cosiddetto 'animale fantastico' o 'Drago dei Vestini', ${ }^{31}$ ben noto per il contesto medio-adriatico e fortemente datante. È verosimile, dai residui che si notano anche sul disco corazza frontale, che la stessa decorazione dovesse esser presente anche sull'altro, ma le tracce sono molto più labili e poco ricostruibili. Quest'ultima caratteristica ci conferma anche che il guerriero, una volta caduto o abbattuto, fosse caduto supino e per un periodo sia rimasto esposto alle intemperie, ragione per cui il retro ha conservato meglio le tracce di colore. Il fatto poi che gli ossidi, che contraddistinguono il colore rosso, non si siano invece del tutto dilavati sul fronte, ci può suggerire che la statua non debba comunque essere rimasta scoperta troppo a lungo, altrimenti il colore sarebbe svanito del tutto.

Attualmente si stanno analizzando anche le tracce di abrasione del nome sull'iscrizione, per capire le modalità con cui sia stata eseguita, non solo per mera documentazione, ma anche al fine di indagare la ratio di questa sorta di damnatio memoriae, oltre a comprendere con quale strumento sia stata eseguita. È ancora in fieri l'analisi delle linee di frattura alle caviglie e alla base dei pilastrini per confermare o

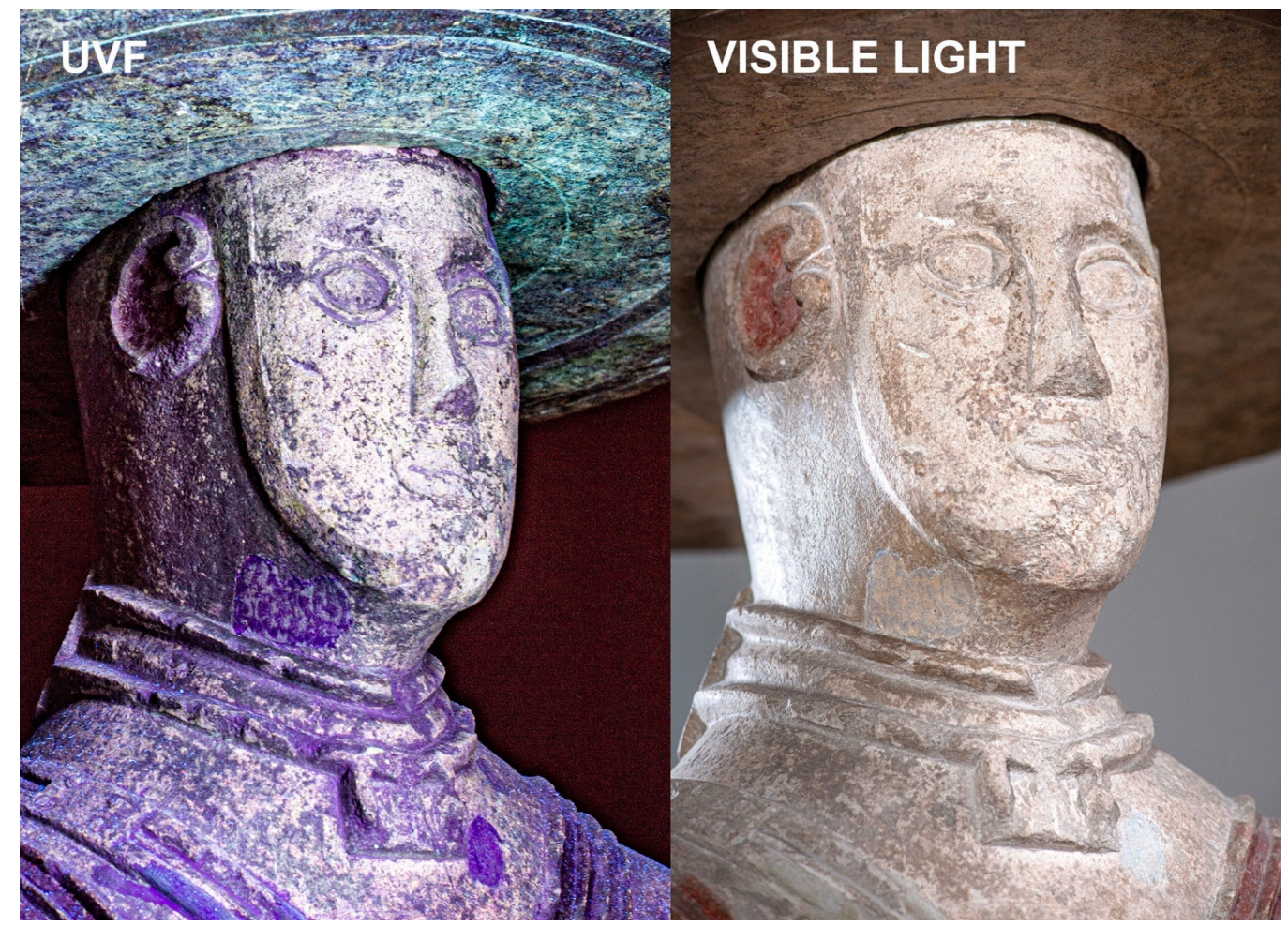

Fig. 8 Il volto del guerriero di Capestrano fotografato sia in luce visibile sia in sfruttando la fluorescenza indotta dalla luce UV. Si notano le zone che contengono una maggiore quantità di colore bianco e in particolare la maschera sul volto.

confutare l'ipotesi di un deliberato 'abbattimento' in antico della statua per motivi di tipo ideologico, politico o tribale. Certo ad una indagine preliminare le fratture sembrano indicare un deliberato intento distruttivo che abbattesse le sculture ma senza distruggerle.

${ }^{31}$ TOMEDI 2000; WEIDIG 2016, pp. 247-272. 

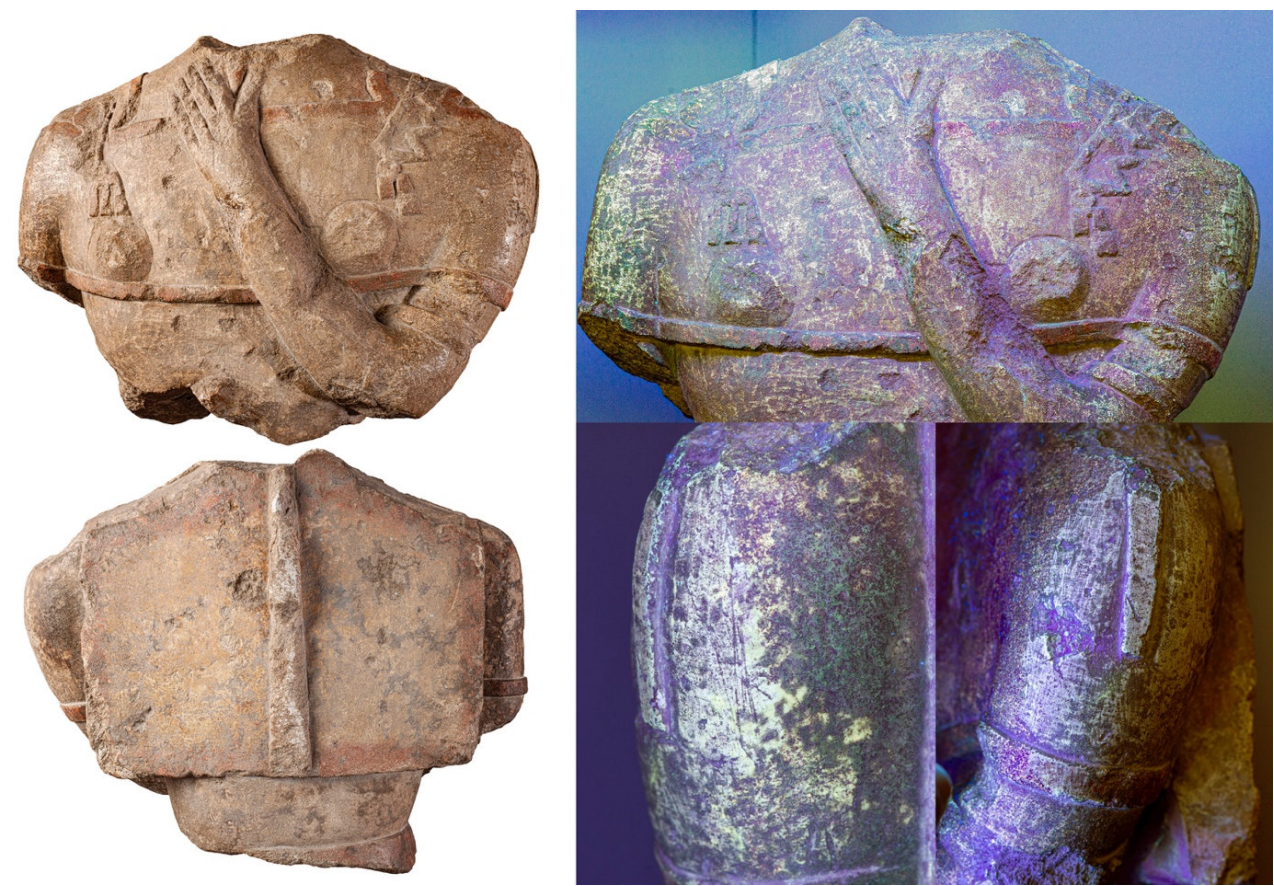

Fig. 9 La Dama di Capestrano, mostra tracce evidenti di colore sia nelle foto in luce visibile e sia nelle immagini effettuate con la fluorescenza indotta dalla luce ultravioletta.

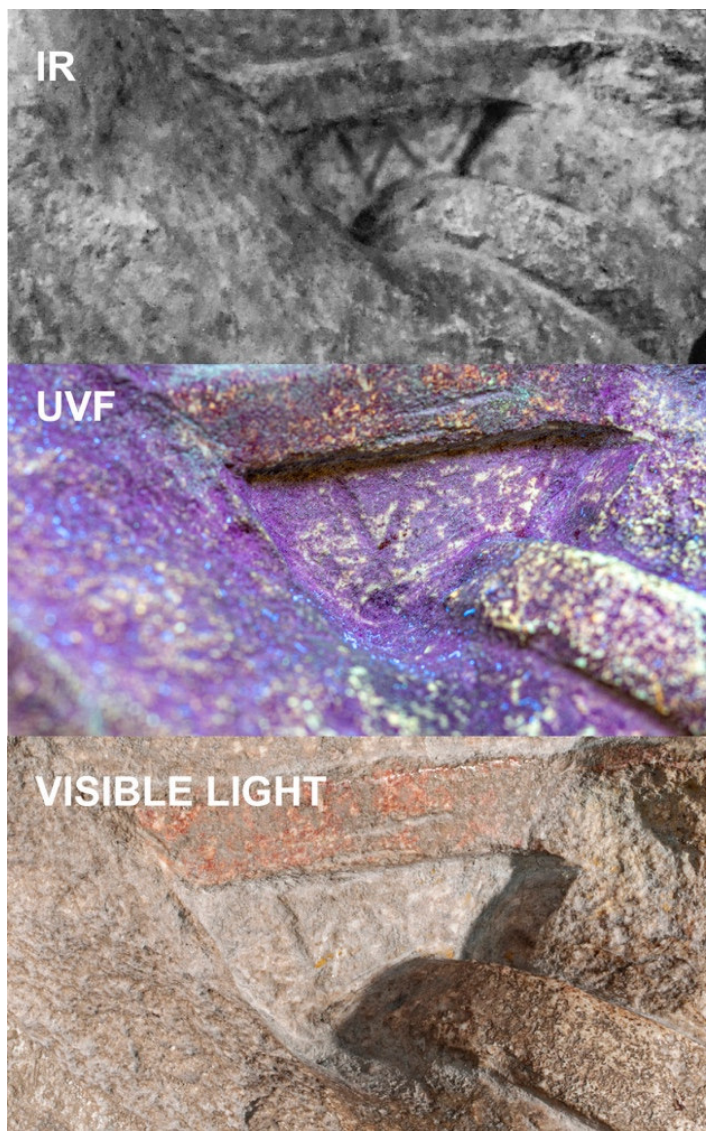

Fig. 10 Il particolare della Dama di Capestrano fotografato utilizzando diversi spettri luminosi, mostra chiaramente la presenza di colore e di una decorazione a zig zag.
Non lontano dal Guerriero era stato rinvenuto anche un torso frammentario di statua femminile (Fig.9), forse di adolescente, probabilmente pertinente al medesimo contesto del Guerriero e di altri frammenti rinvenuti presso le sepolture riconducibili ad un unico gruppo aristocratico di appartenenza (cfr. infra il contributo di V. d'Ercole). La scultura presenta tracce di colore molto meglio conservate della statua del Guerriero. Le cromie della veste, che ad indagine autoptica sembravano limitarsi al rosso e al bianco panna/giallo chiaro, con le indagini con infrarosso e ultravioletto sono più nettamente distinguibili, evidenziando l'utilizzo di una tricromia ben evidente per vesti ed ornamenti, con il bianco delle vesti e del fermatrecce, il giallo, per parti della veste e il rosso per bordature, cintura e ornamenti, come le fibule e i pendagli. L'incarnato, invece, è reso con un ulteriore colore che risulta essere un bianco meno accentuato, colore che non si viene esaltato in fase di riflettanza all'ultravioletto, probabilmente 
perché il bianco era misto ad ossidi per rendere l'incarnato più realistico. Anche in questo caso saranno dirimenti le analisi chimiche in situ e non invasive, mediante le quali sarà possibile conoscere la composizione specifica di ciascun colore.

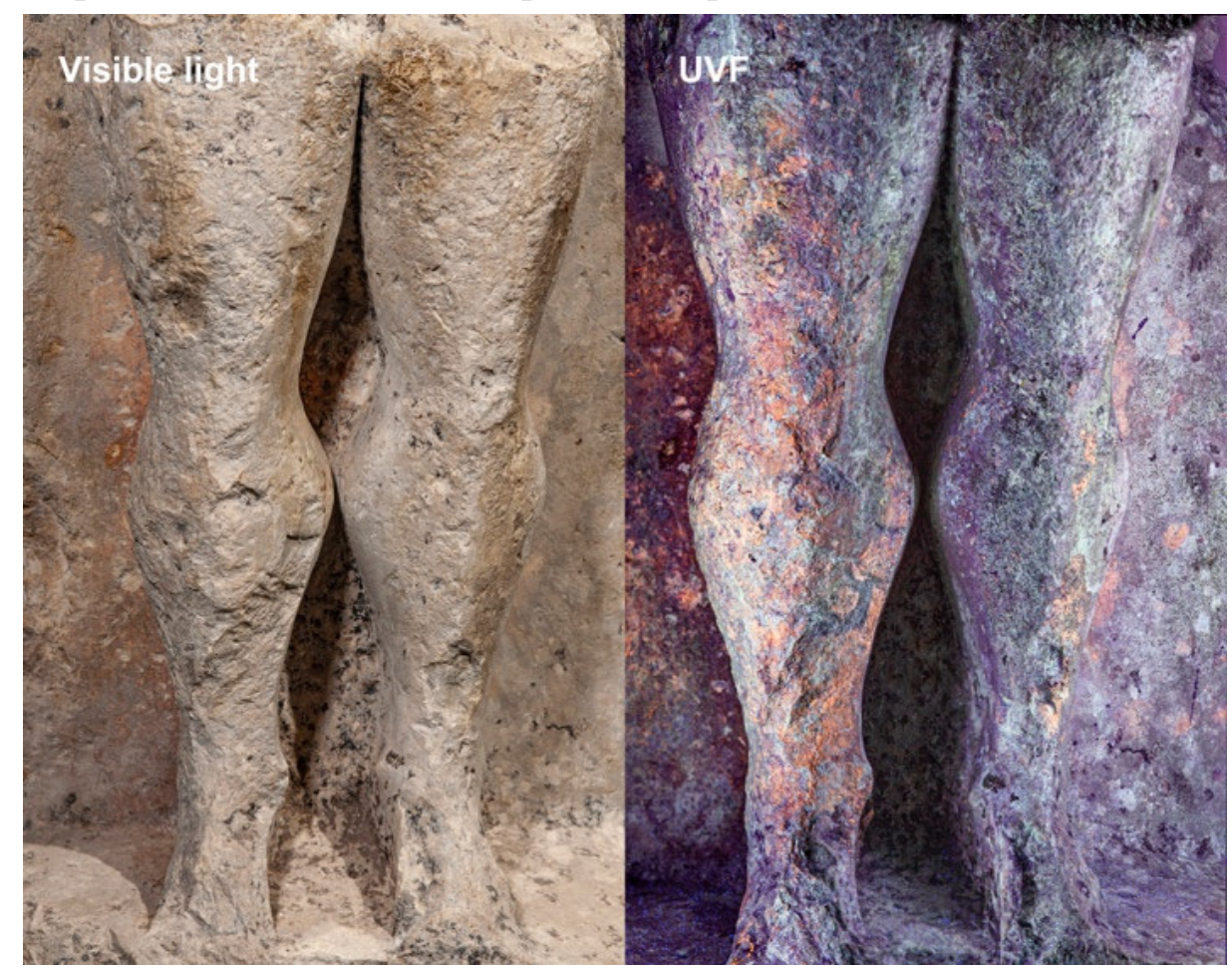

Fig. 11 Le gambe del diavolo, particolare degli schinieri fotografati sia in luce visibile e sia in UVF.

L'analisi delle superfici mediante immagini sia ad alta definizione che ad infrarosso ha evidenziato tracce di decorazione delle vesti: in particolare una fascia in bianco con incisioni a zig zag sotto il seno (Fig.10), e possibili decorazioni geometriche sulle vesti.

Un altro reperto che sta fornendo notevoli risultati dalla correlazione delle analisi geoarcheologiche e fotogrammetriche, è la scultura frammentaria da Collelongo, nota come 'Gambe del Diavolo'. La resa spigolosa degli stinchi e la differenziazione cromatica che sembra risultare dalle indagini multispettrali fanno ipotizzare che il guerriero ritratto vestisse degli schinieri (Fig.11). Se ciò fosse confermato da ulteriori analisi sarebbe anche utile come confronto per lo stesso Guerriero di Capestrano. Le indagini archeometriche da microscopia digitale sembrano confermare tale ipotesi, dato che è distinguibile una differenziazione cromatica dell'area degli schinieri dal resto della statua, che dallo spettro del colore farebbe supporre un verde o un bruno. Bisogna sempre ricordare che $\mathrm{i}$ colori attuali risultano comunque frutto di ossidazioni e variazioni dovute all'esposizione, pertanto non è indicativo in questo caso il colore, ma il fatto che esista una differenza chimica e cromatica tra l'area dei presunti schinieri ed il resto delle gambe.

Inoltre, le indagini hanno potuto documentare e confermare come tutta la scultura fosse colorata, compreso il fondo della stele su cui è realizzata in alto rilievo la figura, ed il retro, che presentano tracce di un'uniforme colorazione in rosso.

Ulteriori risultati ed approfondimenti sono presentati nei contributi che seguono. Le analisi sono ancora in corso e alcuni step del protocollo sono ancora in via di 
definizione; di fondamentale importanza, inoltre, saranno i dati comparativi tra le diverse sculture, che ci permetteranno di tarare i risultati anche in base al substrato litico.

Quando tutte le analisi saranno completate, i risultati complessivi andranno a convergere in schede che raccoglieranno i dati archeologici, storico-artistici, analitici, tecnici, al fine di fornire un quadro quanto più esaustivo possibile su ciascuna delle sculture in una interpretazione basata su un approccio interdisciplinare.

\title{
Gli studi pregressi per la ricostruzione dei contesti: alcuni esempi
}

\author{
Valentina Belfiore ${ }^{32}$ e Maria Emilia Masci $^{33}$
}

Sullo studio della scultura preromana pesa inevitabilmente la difficoltà di ricostruire il contesto archeologico, spesso irrimediabilmente perduto a causa della fortuità di molti rinvenimenti, talvolta anche accompagnati da scavi effettuati in tempi in cui la rigorosità del metodo stratigrafico era ancora lontana dall'essere affermata. Il tentativo di ricostruire un quadro più completo possibile dei rinvenimenti riunendo quanto noto da fonti diverse può tuttavia essere sistematizzato e portare, se non novità sull'argomento, quanto meno nuovi spunti di riflessione sulla contestualizzazione delle opere scultoree e, in alcuni casi a far emergere alcuni elementi inediti. Di tale studio attualmente in corso saranno di seguito presentate alcune questioni a titolo meramente esemplificativo. Come sopra ricordato, l'insieme delle informazioni ottenute dalla ricerca d'archivio, catalografica e bibliografica, confluirà nelle schede predisposte per ogni reperto.

Uno dei primi rinvenimenti ricordati in letteratura, tanto prestigioso quanto segnato dalla scarsità di dati puntuali dal punto di vista dell'attribuzione stratigrafica, è quello del guerriero di Capestrano, che venne alla luce nel 1934 in parti e nel corso di alcuni mesi in località Cinericcio, tra il Lago, le sorgenti di Presciano, e le sorgenti del Tirino (Capo d'Acqua), in seguito allo scasso di un vigneto ad opera del contadino M. Castagna. ${ }^{34}$ V. Cianfarani parla del rinvenimento dapprima del busto (dalle ginocchia in su), quindi del copricapo in parti, così come in frammenti erano anche $i$ due pilastri, mentre la base coi piedi calzati costituiva un'ulteriore blocco. ${ }^{35}$ Secondo un precedente resoconto di Cianfarani, il contadino autore del rinvenimento urtò con la vanga un "insolito sasso" nel quale "nonostante l'armeria [scil. la panoplia raffigurata] non aveva ravvisato l'armigero" ${ }^{36}$ Insieme al "sasso" ne fu trovato "un altro minore e un gran disco", che dopo circa un mese erano quasi affondati in una buca praticata per piantarvi una vite. ${ }^{37}$ Secondo il racconto di Cianfarani fu poi il comandante della locale stazione dei carabinieri a riconoscere nel "sasso" la scultura di un guerriero e ad avvertire le autorità dell'importante rinvenimento. ${ }^{38}$ Stando alle notizie riportate nella scheda di catalogo relativa, fu invece grazie all'interessamento di padre D. Egizi che

\footnotetext{
${ }^{32}$ Direttrice del Museo Archeologico Nazionale d'Abruzzo, Villa Frigerj (MANdA).

${ }^{33}$ Funzionario Archeologo del Museo Archeologico Nazionale 'La Civitella' (MAN).

${ }^{34}$ Scheda di catalogo n. 23861; MORETTI 1936-37; CIANFARANI 1968 ; si veda ora inoltre il volume

D'ERCOLE, ACCONCIA, CESANA 2018.

${ }^{35}$ Cianfarani 1968.

${ }^{36}$ CIANFARANI 1959 (2008).

${ }^{37}$ Ibid., 34.

${ }^{38}$ Ibid., 34.
} 
gli scavi della Soprintendenza Archeologica di Roma diretti da G. Moretti vennero intrapresi fra il settembre e il dicembre del 1934 portando all'individuazione di alcune tombe. ${ }^{39}$

Evidentemente per il diretto coinvolgimento della soprintendenza di Roma, la prima disposizione relativa alla scultura fu quella di trasportarla nel locale Museo delle Terme. ${ }^{40}$ Le misure adottate in tempi di guerra per le sculture conservate in questo museo prevedevano fra l'altro la messa in sicurezza di molte delle statue classiche -

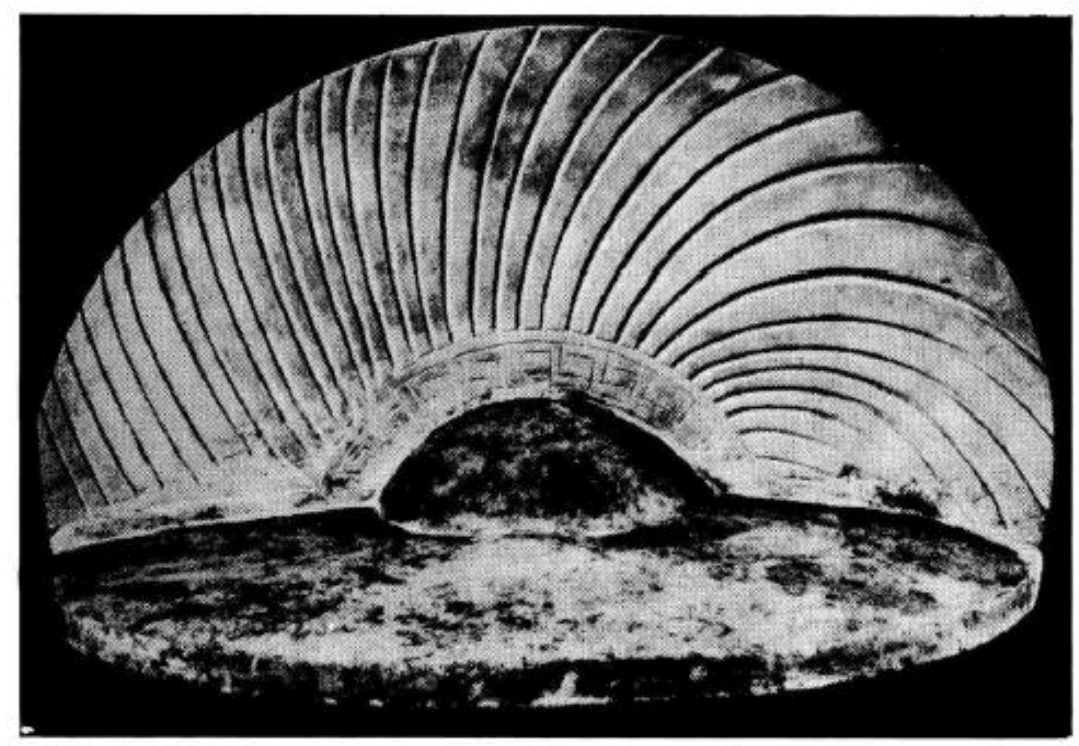

Fig. 12 Il copricapo del guerriero di Capestrano nel primo restauro (da MORETTI 1936)

compreso il guerriero - entro dolii: nello specifico, a quanto ricorda Cianfarani, il guerriero fu nascosto con l'Afrodite di Cirene e sotterrato non lontano dall'edificio. ${ }^{41}$ Al termine della guerra, la scultura venne nuovamente trasferita al Museo Etrusco di Villa Giulia per breve tempo prima di tornare nuovamente in Abruzzo nella sede di Chieti.

La rapidità con cui volle essere presentata la sensazionale scoperta ha condizionato anche la sua restituzione, che fu completata senza molti scrupoli anche nelle parti che risultavano lacunose. Tra le integrazioni riconosciute come più vistose, ${ }^{42}$ sono in particolare il completamento dell'elmo (Fig. 12, che fu anche definito clipeus pinnatus $^{43}$ ) e dei calzari, vezzosamente dotati, mediante tasselli, di due nappine che formavano un fiocchetto sul collo del piede. ${ }^{44}$ In tema di restauri, non si può non citare l'intervento descritto dallo stesso Pico Cellini, famoso restauratore romano chiamato a valutare la scultura conservata a Chieti. ${ }^{45}$ La necessità di revisionare il precedente intervento secondo le indicazioni dello stesso Cellini era stata dettata dalla

\footnotetext{
39 BRENDEL 1935, 572-581; MORETTI 1936-37; le indagini archeologiche effettuate sull'area del rinvenimento sono riepilogate da D'ERCOLE, CELLA in RUGGERI 2007, 33 ss.

${ }^{40}$ L'opposizione dettata dalla decisione di portare il guerriero a Roma è descritta da CIANFARANI 1959 (2008), 34-35.

${ }^{41}$ CIANFARANI 1959 (2008), 35.

42 CIANFARANI 1968.

${ }^{43}$ FERRI 1949.

${ }^{44}$ CIANFARANi 1968, 10: lo stesso parla di calchi in gesso eseguiti sulla statua dopo la sua integrazione (ibid., figg. 8-11; cfr. infra, Fig. 27), attualmente non rintracciabili.

${ }^{45}$ CELLINI 1992, 136-137; figg. 14-15.
} 


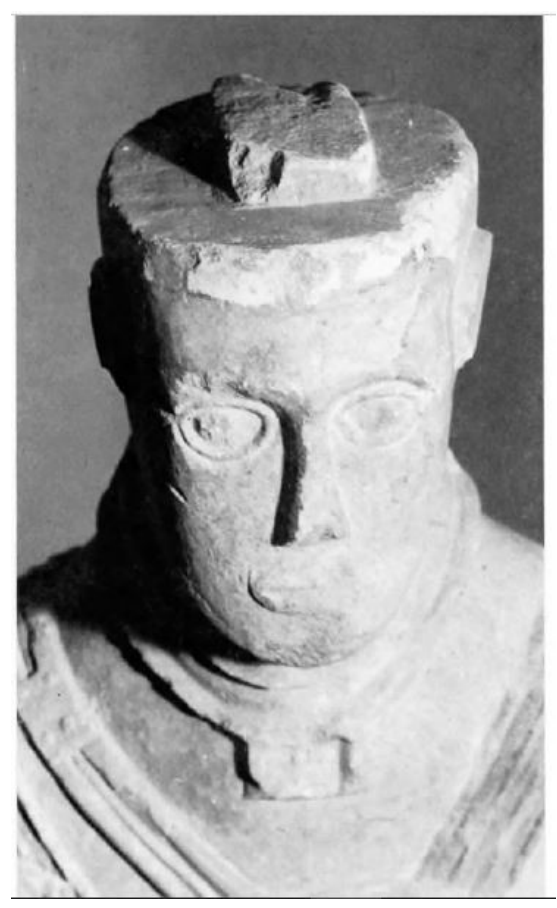

Fig. 13 La testa del guerriero priva dell'elmo (da CIANFARANI 1966, 4, fig. 19).

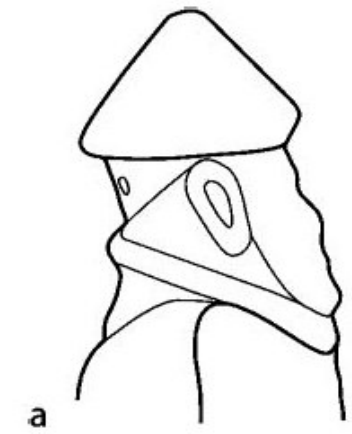

partecipazione del guerriero alla mostra "Antiche Civiltà d'Abruzzo" organizzata a Roma nel 1969. ${ }^{46}$ Come Cellini ricorda, tanto il copricapo, quanto i calzari, frutto di una sovrainterpretazione del monumento, sono stati smontati. La superficie, ricoperta da uno strato grigio di calcare formatosi anche a causa dei calchi effettuati sulla statua, fu pulita e riportata ai colori del rosso, che emergevano in molti punti. ${ }^{47}$ Tale colorazione era ottenuta, stando al Cellini, "in propora di murice in veicolo ceroso" 48 o, come si espresse Cianfarani, in biossido rosso di ferro e fu ravvivato chimicamente mediante una soluzione alcolica di nylon. ${ }^{49}$

Fra le discussioni che in letteratura hanno interessato il guerriero, un tema è rappresentato sicuramente dalla presenza o assenza di una maschera sul viso e sul suo significato (Fig. 13). Con poche eccezioni, gli studiosi che si sono interessati della scultura (da Cumont, a Moretti a Cianfarani, per ricordarne alcuni, fino a Schickler in tempi più recenti) hanno rilevato la presenza di una maschera, secondo alcuni anche dotata di

Fig. 14 Riproduzione delle maschere dei guerrieri di Hrischlanden (a) e Capestrano (b), da ARMIT, GRANT 2008, 413, fig. 4.

protezione per le orecchie. ${ }^{50}$ L'unica voce in senso contrario è stata quella di S. Ferri, che nel suo intervento del 1949 osservava per inciso "Lascio da parte la maschera e il para-orecchi, di cui nessuno, credo, parla più". ${ }^{51}$ Su questa scia più di recente si sono

\footnotetext{
${ }^{46}$ Informazioni riportate nella scheda di catalogo n. 23861.

${ }^{47}$ CELlini 1992, 136-137.

${ }^{48}$ CELLINI 1992, 136.

${ }^{49}$ Cianfarani 1968, 14. Per indagini recenti cfr. S. Agostini 2010 in FranCHI DELL'Orto, La Regina, BUONOCORE 2010, 226-229.

${ }^{50}$ CUMONT 1935; MORETTI 1936-37, 109-114; CiANFARANi 1969, 78; CIANFARANi 1976, 76; SCHICKLER 1999 in Piceni 1999, 25-26.

${ }^{51}$ FERRI 1949, 1.
} 
pronunciati V. d'Ercole (v. infra) e L. Franchi dell'Orto, la quale ha considerato la presenza di maschere su rappresentazioni di guerrieri italici come illusoria e derivata dal trattamento plastico dei volti. ${ }^{52}$ Di fatto tuttavia, la macrofotografia ha dimostrato che il guerriero di Capestrano indossa una maschera, come pare probabile anche per la testa di Manoppello (v. supra, il contributo di O. Menozzi e il contributo collettivo su metodologie e risultati preliminari). Allo stesso modo un profilo continuo a rilievo che si osserva sul volto del guerriero di Hirschlanden e che continua anche al di sopra degli occhi, ha indotto gli studiosi a riconoscere tale sagoma come maschera a rilievo (Fig. 14) ${ }^{53}$ Per quest'ultimo è stato anche richiamato il confronto con la maschera di bronzo della tomba hallstattiana di Keinklein in Stiria, datata al VII sec. a.C. ${ }^{54}$ Una maschera funeraria è stata chiamata in causa anche per il cippo di San Giovanni in Persiceto (Bologna), cilindrico e decorato unicamente da una fascia a rilievo a metà altezza e superiormente da un volto barbato. ${ }^{55}$ Trovato nel 1891 a breve distanza da una tomba femminile e da un'altra tomba orientalizzante, il cippo è stato attribuito al VII sec. a.C. per confronti con i volti che ornano i canopi etruschi. ${ }^{56}$ Per analogia si possono considerare anche i volti allungati riprodotti su due delle tre stele di Penna Sant'Andrea, queste ultime di cronologia decisamente più recente (cfr. V. d'Ercole, infra). Le provenienze da contesti funerari, malgrado l'assenza di dati contestuali più specifici, induce a ritenere tanto il monumento scultoreo quanto la maschera in particolare come espressione di una monumentalizzazione a scopo funerario del defunto, piuttosto che di un antenato: i dettagli talvolta puntuali (ad es. la resa calligrafica delle ciocche della barba nel cippo di San Giovanni in Persiceto e il lungo mento dei volti di Penna Sant'Andrea, interpretabili come barbe) lasciano infatti pensare che ad essere riprodotto sia un individuo reale, non idealizzato.

Diversamente dal guerriero, la dama di Capestrano ha ricevuto un'attenzione più contenuta. Come ricordato da V. d'Ercole (infra), le uniche notizie che concernono il suo ritrovamento riguardano la sua posizione, sotto il copricapo del guerriero nello stesso anno in cui lo scavo ebbe luogo. ${ }^{57} \mathrm{Il}$ busto risultava più piccolo e frammentario ma non meno interessante dal punto di vista della conservazione di tracce di colore, per il tipo di abbigliamento e di ornamenti indossati, oltre che per il soggetto femminile stesso, eccezionalmente rappresentato in forme scultoree. ${ }^{58}$ In base agli scarni elementi a disposizione, la cronologia della dama, come pure del guerriero, si è finora basata principalmente su dettagli stilistici e di costume. ${ }^{59}$ Le nuove indagini si rivelano dunque

\footnotetext{
52 FrANCHI DELL'ORTO in FrANCHI DELL'ORTO, LA REGINA, BUONOCORE 2010, 188.

${ }^{53}$ SCHICKLER 1999, 25-26; MARZOLI 2003, 204. Sulla maschera del guerriero di Hirschlanden si veda già ZÜRN 1964, 225; lo stesso successivamente si dichiara più cauto (ZÜRN 1970, 67).

${ }^{54}$ EGG 1994; EGG 2004.

${ }^{55}$ MARCHESI 2011, 48-50, 194-195, 281-282, tavv. 12-13.

56 Ibid.

${ }^{57}$ MORETTI 1936, 14 ss.

${ }^{58}$ Moretti 1936, 14 ss.; COlONNA, PERONI 1961, 261; CiANFARANi 1966, 6; CiANFARANi 1969, 77 ss. n. 181; CIANFARANI 1970, 121, 172 ss.; CIANFARANI 1976, 76 ss.; CIANFARANI, FRANCHI DELL'ORTO, LA ReginA 1978, 79, 118; 185 ss.; 314 ss.; MARINANGELi 1978, 8 ss.; PAPI 1981, 16 ss., nota 4, 7. Soggetti femminili su stele funerarie a rilievo ad es. nel comprensorio felsineo compaiono solo in un secondo momento (GOVI 2014, 139, nota 55).

${ }^{59}$ La datazione della dama alla fine del VI sec. a.C. è stata inizialmente proposta sulla base delle due fibule ad arco serpeggiante a tre gobbe ad angolo acuto ornate da pendagli trapezoidali a due denti, che reggono il corpetto, documentate da corredi funerari in tale cronologia. Secondo studi più recenti (WEIDIG 2014, 229-284, con bibliografia precedente), il più ampio range cronologico di tali fibule e in particolare il confronto anche per il tipo di pendenti con una fibula analoga dalla tomba 34 Azzurra di
} 
più che mai cruciali per appurare l'esistenza, sulla dama e sul guerriero, di nuovi elementi per una riflessione globale (vedi quanto anticipato nella sezione sui risultati preliminari e infra il contributo di R. D'Errico).

Ancora più scarne sono le notizie che concernono il rinvenimento di opere scultoree in anni più recenti, come le "gambe del diavolo", trovate in data non meglio precisata alle "stallette" di S. Castro nella conca di Amplero e in seguito inserite nelle mura che cingevano la proprietà F. Lombardi a Collelongo, finché nel 1957 le gambe non furono da qui smontate per essere portate nel Museo di Chieti. ${ }^{60}$

Nel 1971 si è aggiunta un'ulteriore scoperta, anch'essa fortuita, di un torso, identificato come "torso di Atessa" o indicato più di recente come "torso di Pallano". ${ }^{61}$ Stando alle informazioni riportate nelle schede di catalogo, il torso proverrebbe dalla sella di Colledimezzo di Pallano, nel comune di Atessa, ${ }^{62}$ mentre nella ricostruzione più dettagliata di A. Faustoferri il torso risulta trovato alle pendici del colle Archiano, in località Piano San Giorgio, dove i proprietari lo avrebbero sistemato sul limite del loro appezzamento fino al 1971. Durante quest'anno, il torso risultava in possesso di un antiquario di Atessa, Giuseppe Rancitelli, quando Valerio Cianfarani lo individuò e diede avvio alla pratica di acquisizione oltre che a scavi nella zona che gli fu indicata. ${ }^{63}$ Nel terreno indagato furono così trovati i frammenti delle braccia, ancora mancanti, e resti di un selciato la cui funzione non è stata meglio definita. ${ }^{64}$

Di tale quadro ricostruttivo la ricerca è ad uno stadio, come anticipato, ancora preliminare. In tutti i casi ricordati, la scarsità di dati puntuali a disposizione rende tanto più necessario un ulteriore approfondimento dello studio sui reperti mediante $\mathrm{i}$ protocolli indicati in sede introduttiva, che così come per la coppia di sculture di Capestrano, anche per le "gambe del diavolo" e per il torso di Atessa si sono rivelati promettenti (v. supra).

[V.B.]

\footnotetext{
Bazzano, lascia piuttosto ipotizzare una datazione ad un momento avanzato della prima metà del VI sec. a.C. (WEIDIG 2014, 267).

${ }^{60}$ Scheda di catalogo n. 24941; A. FAUSTOFERRI in RUGGERI 2007, 68 ss.; si veda inoltre COLONNA, PERONi 1961, 262; Cianfarani 1966, 5; CiANFARANi 1969, 76 ss. n. 180; CiAnFARANi 1970, 120; CIANFARANI 1976, 80; CIANFARANI, FRANCHI DELL'ORTO, LA REGINA 1978, 79, 116, 351; LETTA 1979, 102 ss.

${ }^{61}$ Per la ricostruzione del contesto archeologico del sito ante scoperta, cfr. COLONNA 1955. Si vedano inoltre CIANFARANI 1975, 51; CIANFARANI 1976, 83; CIANFARANI, FRANCHI DELL'ORTO, LA REGINA 1978, 119, 352; IACUlli 1982, 198; PAPI 1981, 17 ss.; COLONNA 1992, 124.

${ }^{62}$ Scheda di Catalogo n. 23881.

${ }^{63}$ Per un inquadramento più ampio del contesto archeologico cfr. A. FAUSTOFERRI in RUGGERI 2007, 57 ss.

${ }^{64}$ Ibid.
} 
Nel 1980 l'esiguo numero di sculture italiche dell'Abruzzo ad oggi conosciute, è stato arricchito con l'aggiunta della cosiddetta 'Testa di Manoppello' (Fig. 15). Rinvenuta fuori contesto murata nella parete di un'abitazione del paese grazie alla segnalazione del Sig. R. Di Fiore, un appassionato di antichità e storia locale, e successivamente acquisita dallo Stato, è oggi esposta al Museo Archeologico Nazionale "la Civitella" di Chieti ${ }^{65}$. La testa, realizzata in calcare bianco della Maiella di dimensioni leggermente superiori al vero (h. cm. 32,7; diam. cm. 25), faceva parte probabilmente di una scultura a tutto tondo che riproduceva la figura intera di un guerriero, databile tra la seconda metà del VI ed il V sec. a.C. R. Papi ipotizza che il contesto originario di provenienza sia da ricercare poco lontano dal luogo di rinvenimento, forse nell'area della necropoli in contrada Cappuccini, che ha restituito materiali di età arcaica. La scultura è
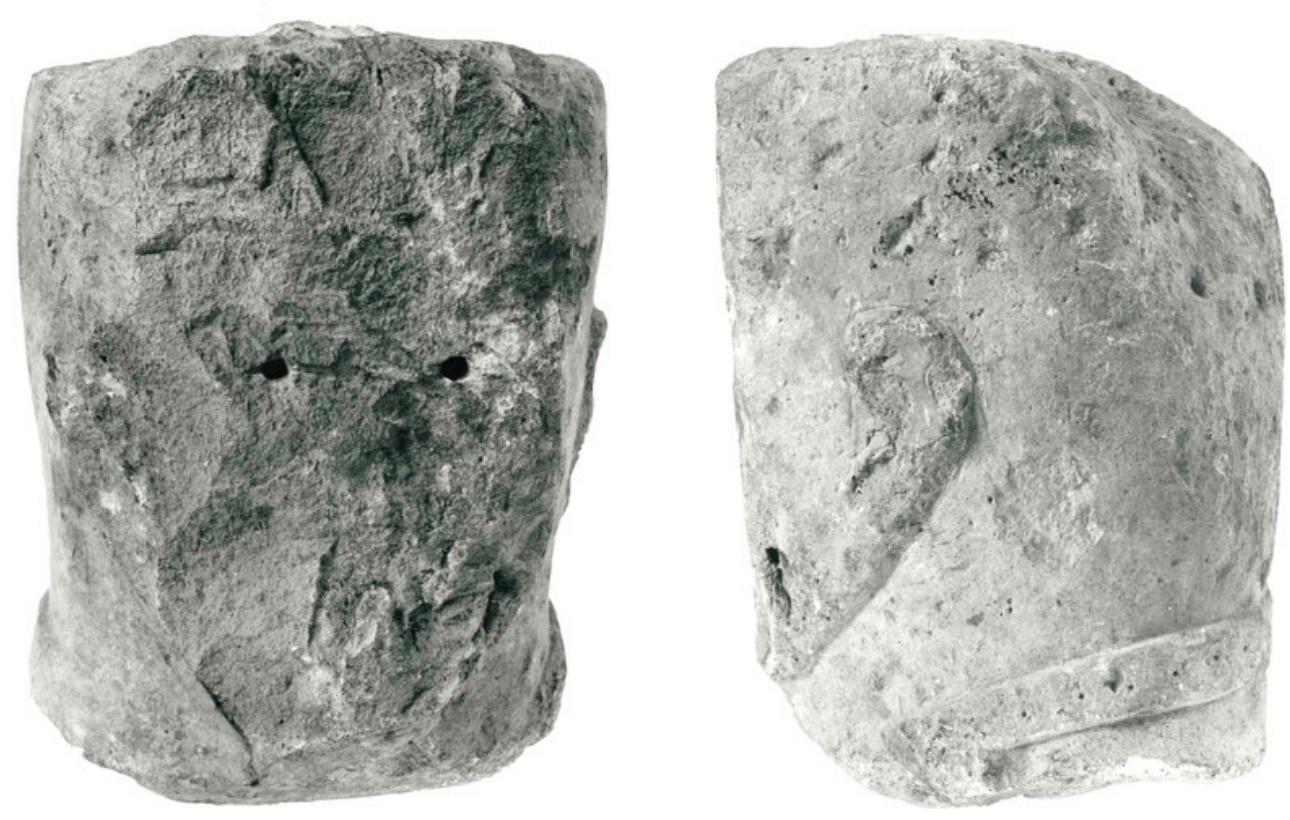

Fig. 15 Cosiddetta 'Testa di Manoppello'.

conformata come un grande blocco cilindrico, troncato all'altezza del collo lungo una linea irregolare. Il collo non è volumetricamente distinguibile dal volto, ma è segnato dalla presenza di un torques inciso, decorato da piccoli cerchi regolari con un punto centrale. Sulla faccia superiore del blocco calcareo è scolpito un dado, utilizzato con tutta probabilità per fissare un copricapo. Il volto appare oggi fortemente danneggiato ed in varie parti poco leggibile: le orecchie sono delineate a rilievo con una netta incisione unita senza discontinuità alla mandibola che sembra proseguire delimitando il volto in basso da un lato all'altro con una linea ininterrotta. La parte frontale del viso è completamente scalpellata seguendo un piano inclinato di circa $7^{\circ}$, forse per effetto di un'operazione di 'damnatio memoriae' analoga a quella che è stata ipotizzata per altre sculture italiche, oppure per motivi di reimpiego. L'attenzione di molti studiosi si è concentrata sui due profondi fori evidenti nella sede degli occhi, che R. Papi ha

\footnotetext{
${ }^{65}$ Chieti, MAN "la Civitella", inv. 24097, scheda di catalogo n. 1300024883, neg. N. 28022-28027. Sul rinvenimento e per l'inquadramento storico-archeologico cfr. PAPI R.1981 e PAPI R. 1986, pp. 136-139, nota n. 59 .
} 
interpretato come fori di alloggiamento per dei perni utilizzati per fissare una maschera probabilmente metallica, mentre altri hanno spiegato come interventi successivi dovuti al reimpiego del blocco o ancora come agganci per inserti in altro materiale. Le analisi da condurre su questo pezzo, ancora totalmente da svolgere, saranno certamente utili a raccogliere dati ed informazioni per meglio comprendere il contesto, la funzione, l'inquadramento storico-artistico e culturale di quest'opera il cui pessimo stato di conservazione e la cui assenza di dati sul contesto originario lasciano oggi molti interrogativi ancora aperti.

[M.E.M.]

\title{
Una 'storia spezzata': tentativo di ricostruzione dei contesti di Capestrano e Penna Sant'Andrea
}

\author{
Vincenzo d'Ercole ${ }^{66}$
}

La maggior parte della scultura preromana in Abruzzo risulta priva del contesto archeologico: in molti casi i singoli pezzi sono stati rinvenuti 'casualmente' in una collocazione secondaria (come materiale di reimpiego in una costruzione: Manoppello e Collelongo) o, addirittura, acquistati dagli 'occasionali' scopritori come Atessa ${ }^{67}$. Gli unici materiali che provengono da scavi archeologici regolari sono i due contesti, probabilmente i più significativi della serie, di Capestrano e di Penna Sant'Andrea ${ }^{68}$. Ma le buone notizie si esauriscono qui perché, come vedremo di seguito, delle due campagne di scavo condotte da Giovanni Annibaldi a Capestrano, nel 1934 e nel 1937, la seconda è rimasta inedita ${ }^{69}$; stesso destino per lo scavo, diretto da Adriano La Regina, a Penna Sant'Andrea nel 1974. Lo scavo del 1934 a Capestrano, condotto in seguito al rinvenimento fortuito della statua del Guerriero, che portò all'acquisizione di altre parti della statua maschile e della porzione superiore della statua femminile, non fu pubblicato dallo scavatore, Annibaldi, ma dal suo Soprintendente ${ }^{70}$ con particolare riferimento alle sculture in pietra che avevano suscitato tanto scalpore ${ }^{71}$. Della campagna di ricerche effettuata nel 1937 nella stessa area, nel corso della quale furono portate alla luce un'altra statua maschile munita della coppia di pilastrini e recante una iscrizione della stessa tipologia di quella già emersa oltre alla parte inferiore della statua femminile, non è stato pubblicato nulla e non sono disponibili i diari di scavo o la documentazione fotografica $^{72}$. Le circostanze assumono contorni drammatici perché delle due statue, trasferite nel 1945 dal Museo Nazionale Romano a quello Etrusco di Villa Giulia, si sono perse le tracce ${ }^{73}$. Nel corso dell'anno 1992 la Provincia de L'Aquila effettuò dei lavori per la realizzazione di un impianto irriguo che, sul finire, nel mese di settembre, furono bloccati dalla Soprintendenza Archeologica dell'Abruzzo che

\footnotetext{
${ }^{66}$ Archeologo, già Direzione Generale Archeologia MIBCT, Docente di Etruscologia e Civiltà dell’'Italia preromana presso l'Università G. d'Annunzio di Chieti.

${ }^{67}$ D'ERCOLE 2014, pp. 463-474.

${ }^{68}$ D'ERCOLE 2017, pp. 223-238. La testa di Loreto Aprutino proviene da un'area di necropoli, Farina Cardito, dove Giovanni Leopardi portò alla luce 25 sepolture ma senza indicazioni di provenienza o contesto (CIANFARANI-FRANCHI DELL'ORTO-LA REGINA 1978, pag. 29). Verosimilmente dalla necropoli di Comino dovrebbe provenire la stele di Guardiagrele recuperata, occasionalmente, nel 1965.

${ }^{69}$ CELla 2012, pp. 57-105.

${ }^{70}$ MORETTI 1936/7, pp. 94-114.

${ }^{71}$ MORETTI 1936.

${ }^{72}$ D'ERCOLE, ACCONCIA, CESANA 2018.

${ }^{73}$ D'ERCOLE, ACCONCIA, CESANA, MENOZZI in c.s.
} 
effettuò, nell'area di Capo d'Acqua, lo scavo di emergenza di tre sepolture arcaiche, il recupero di manufatti neolitici e di otto frammenti di un letto funebre rivestito in osso lavorato ${ }^{74}$. Nel dicembre dello stesso anno la casa d'aste Sotheby's, sede di New York, vendette, ad un privato, una porzione di statua in pietra simile al Guerriero di Capestrano per 231.000 dollari che recava, come indicazione di provenienza, 'central Italy $^{75}$. Non avendo mai potuto usufruire di una visione autoptica della statua commercializzata nel 1992 e non avendo potuto effettuare su di essa alcun tipo di analisi rimane impossibile stabilirne l'autenticità ma appare chiaro che ai piedi della tomba $3^{76}$ di Capestrano vi fossero tre o quattro statue di pietra di cui una femminile mentre le altre (due o tre) di sesso maschile e della medesima tipologia. Le poche testimonianze coeve di statuaria in pietra di ambito etrusco mostrano sempre un gruppo di personaggi sia maschili che femminili: probabilmente sei nel tumulo orientalizzante della Pietrera a Vetulonia ${ }^{77}$ e almeno due nel caso del recupero effettuato dai Carabinieri Tutela Patrimonio Culturale nel 1987 nella tomba C della necropoli di Casa Nocera a Casale Marittimo presso la foce del Cecina, nel territorio di Volterra ${ }^{78}$. Quattro o cinque sono i personaggi, di ambedue i sessi, rappresentati nelle statue-stele di Gozzo Veronese fra Etruria Padana e mondo veneto ${ }^{79}$. L'Abruzzo ha una lunga storia di rappresentazioni scultoree in pietra 'corali' che affonda le sue radici tra l'età del Bronzo Finale 3 e la Prima età del Ferro (XI-VIII sec. a. C.) con i menhir rinvenuti, sempre in accurati e documentati contesti di scavo, lungo la dorsale appenninica, nelle necropoli con tombe a tumulo di Celano-Paludi, Scurcola Marsicana-Piani Palentini, Fossa-Casale, L'Aquila-Bazzano e, probabilmente, San Pio delle Camere-Colli Bianchi. Il modello del guerriero armato di tutto punto effigiato nei due o tre esemplari di Capestrano, verosimilmente dallo scultore Aninis, era stato preceduto dalla statua, armata di pugnale, di Rapino e da quelle di dimensioni maggiori del vero di Manoppello e di Numana ${ }^{80}$ fra i Piceni ${ }^{81}$. Probabilmente Aninis riprende la tecnica dei pilastrini laterali, già presenti nella statua di Atessa (anch'essa di dimensioni maggiori del vero), associandola all'iconografia dell'eroe in armi. Il tema del 'Signore della guerra' non trova riscontro nelle poleis dell'Etruria mentre appare dominante nelle comunità etnicoterritoriali dell'Europa hallstattiana prima e celtica poi come aveva ipotizzato nel 1949 Silvio Ferri ${ }^{82}$ su basi stilistiche e non socio politiche ed ideologiche.

\footnotetext{
${ }^{74}$ USAI 1993, pag. 299.

${ }^{75}$ D’ERCOLE 2015, pp. 99-111.

${ }^{76}$ La tomba 3 si componeva di una fossa contenente i resti di un individuo adulto armato di spada lunga in ferro affiancata da un loculo, privo di inumato, con un ricco corredo di armi in ferro, vasi di bronzo, strumenti in metallo: un cenotafio di un personaggio illustre? Il complesso sepolcrale è l'unico in tutta la necropoli con orientamento verso nord (la prassi del periodo arcaico è l'orientamento ad est) ed appare circondato da seppellimenti radiali che fanno ipotizzare la presenza di un tumulo. Il sepolcro è posizionato sul margine della via sacra che collegava la necropoli all'abitato.

${ }_{77}$ MagGiani 2015, pp. 197-221.

${ }^{78} \mathrm{La}$ statuaria in pietra tirrenica, etrusca era pensata per essere al chiuso, riservata ai soli fruitori del sepolcro (si pensi alla manifestazione più antica: la tomba delle statue di Ceri) mentre quella Adriatica è fatta per stare all'aperto, sempre in contesti funerari, ma visibile a tutti e non ai soli frequentatori della specifica tomba a cui i gruppi statuari fanno riferimento.

${ }^{79}$ MALNATI 2002, pp. 127-138; GAMBA, GAMBACURTA 2011, pp. 159-193.

${ }^{80}$ LA REGINA 2010, pp. 230-273. Il fenomeno del 'gigantismo' nella produzione delle statue anyiche (si pensi all'esercito di Monte Prama in Sardegna) non è legato alla maggiore antichità di realizzazione delle opere, ma alla necessità di fungere da deterrente psicologico per incutere timore nei confronti dei vicininemici; esemplare in questa dinamica il trofeo ligneo. Alto oltre due metri e mezzo, rivestito di corazza e di elmo in bronzo, riferibile al IV secolo a.C., rinvenuto in Magna Grecia e conservato nell'Antikensammlung di Monaco di Baviera (Graells i Fabregat 2018, pp. 159-194).

${ }^{81}$ COLONNA 2001, pp. 104-109; FrANCHI DELL'ORTO 2010, pp. 180-225.

${ }^{82}$ FERRI 1949, pp. 1-9.
} 
Tra le informazioni più salienti che stanno emergendo dalle analisi in corso su questo gruppo scultoreo la principale riguarda il volto del Guerriero che, a differenza del resto dell'incarnato, è colorato in bianco ${ }^{83}$ : questa prerogativa contribuisce, in modo determinante, a corroborare l'ipotesi della presenza di una maschera metallica. Già ma che tipo di maschera e con quale funzione? Credendo alla validità del dato archeologico contestuale mi riesce difficile accettare, supinamente, l'ipotesi della maschera funeraria attestata, in lamina d'oro, nell'età del Bronzo in Grecia, in cera o in altro materiale duttile (secondo alcune fonti) fra etruschi e romani, mai in contesti medio adriatici ${ }^{84}$. La presenza del soprastante elmo ci potrebbe suggerire, per la maschera, un utilizzo come protezione del viso attestata soprattutto negli elmi della cavalleria ${ }^{85}$. Che l'equipaggiamento militare della statua del Guerriero fosse quello consono ad un combattente a cavallo ce lo avevano già indicato l'uso della spada lunga in ferro, da fendente ${ }^{86}$, e soprattutto le due corte $(129$ e $136 \mathrm{~cm}$.) lance da getto caratteristiche dei cavalieri privi da staffe. Quello che appariva come un robusto sottogola, necessario per un elmo così pesante, potrebbe essere, alla luce di queste nuove acquisizioni, il bordo rilevato della maschera facciale: il suo ripiegamento, in prossimità dell'attacco all'elmo, potrebbe suggerire che il frontale fosse basculante permettendo, così, l'alzarsi e l'abbassarsi della visiera solo nel momento della battaglia ${ }^{87}$. Sarà molto interessante, in questa prospettiva, verificare 'prove' dell'esistenza di schinieri e, soprattutto, accertare se il Guerriero indossasse solo quello sinistro ${ }^{88}$ come peculiare dei cavalieri medio adriatici: si veda la tomba 97 di Campovalano ${ }^{89}$. L'altra 'scoperta' di grande interesse è la presenza della decorazione sulla coppia di dischi corazza effigiati sulla statua. Le corazze note da contesto di scavo nel territorio Vestino sono la tomba 1 di Bazzano e la tomba 118 di Fossa: si tratta di due dischi bilobati, ad otto, del tipo Mozzano privi di emblema centrale. ${ }^{90}$ Le nuove indagini hanno rilevato la presenza di un animale mitologico che, anche se deve essere ancora analizzato nei dettagli, appare assimilabile al cosiddetto Drago dei Vestini. ${ }^{91}$ Questo nuovo elemento toglie il mondo dell'archeologia da un grande imbarazzo in quanto sulla raffigurazione scultorea più famosa della regione avevamo un tipo di corazza non attestato nel record

\footnotetext{
${ }^{83}$ Tracce biancastre sono, parzialmente, visibili sulla capigliatura della cosiddetta Dama di Capestrano: ove confermato potrebbe trattarsi di fermatrecce in argento che tenevano in ordine la lunga treccia posteriore della fanciulla pettinata alla moda chiusina.

${ }^{84}$ Come è ben noto l'unico reperto assimilabile a questa, presunta, categoria è la maschera in bronzo ritrovata sporadicamente ed isolata a Fonte del Pidocchio, presso Longano, nel Molise di cui si ignorano contesto, cronologia e funzioni (CECCARELLI, FRATIANNI 2017, pp. 116-117).

${ }^{85}$ Per un contesto molto più recente $(9 \mathrm{~d}$. C. $)$ si potrebbe ricordare la maschera frontale, in argento, di un cavaliere romano, rinvenuta nella foresta di Teutoburgo in Bassa Sassonia; lo strato pittorico bianco, evidenziato dagli ultravioletti, applicato sopra il volto del guerriero potrebbe alludere alla presenza di un manufatto in argento.

${ }^{86}$ D’ERCOLE 2015 B, pp. 245-268.

${ }^{87}$ La presenza di una maschera facciale sotto l'elmo rende, chi lo indossa, indistinguibile dall'esterno. La storia (Baldovino IV d'Angiò, il re lebbroso di Gerusalemme) e la letteratura (il fratello di Luigi XIV di Francia, la Maschera di ferro) hanno attinto a piene mani in questa possibilità: anche la tomba 3 di Capestrano con l'affiancato cenotafio di un ricco guerriero permetterebbe molte ipotesi.

${ }^{88}$ Il lato sinistro del corpo di un cavaliere, con cui manovra il cavallo attraverso le briglie, è quello più scoperto ed indifeso (a meno che non si tratti di un mancino) ed è quindi quello con le maggiori protezioni 'passive': scudo, schinieri. Per non appesantire troppo il carico per il cavallo stancandolo eccessivamente si tendeva ad alleggerire l'equipaggiamento del cavaliere: lo schiniere destro era uno dei complementi dell'armamento più facilmente sacrificabile.

${ }^{89}$ D’ERCOLE 2010. pp. 223-234.

${ }^{90}$ D'ERCOLE 2014 B, pp. 29-61.

${ }^{91}$ TOMEDI 2000; WEIDIG 2016, pp. 247-272.
} 

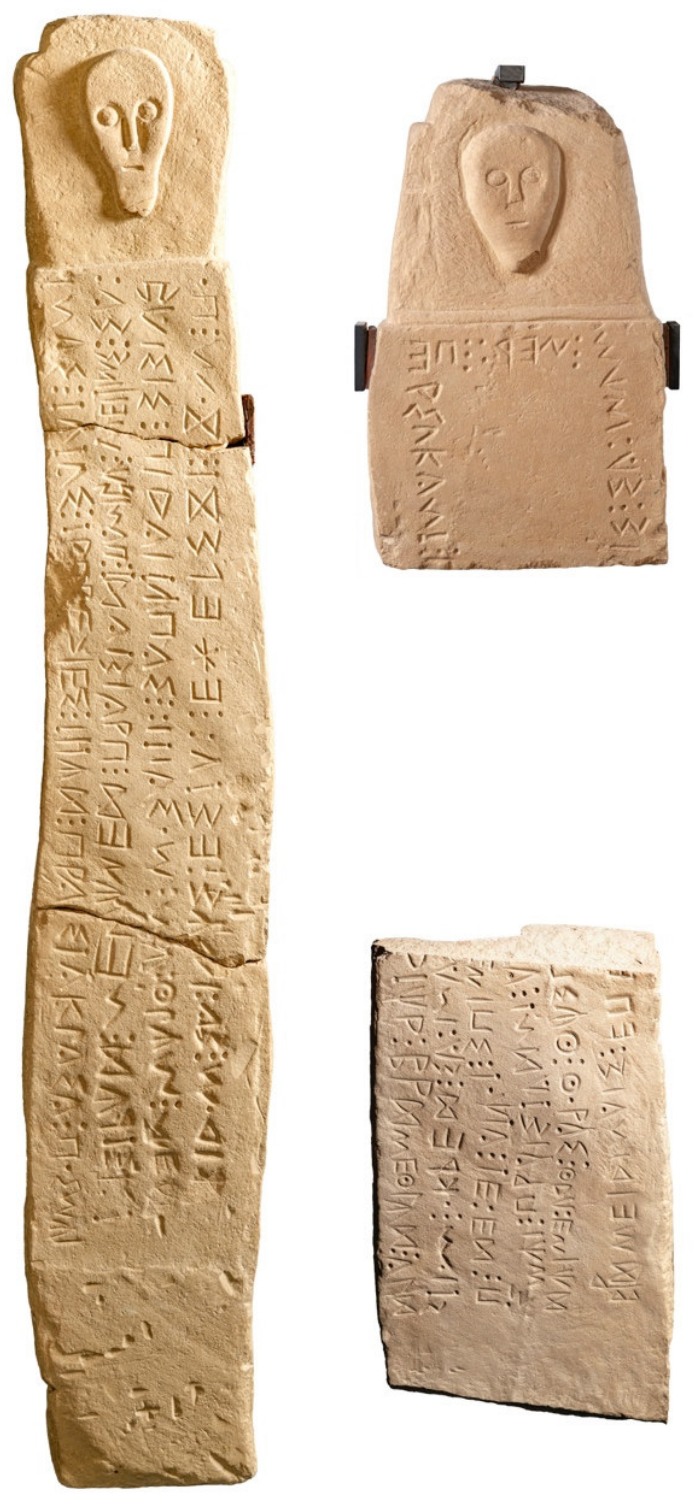

Fig. 16 Le tre Stele di Penna Sant'Andrea.

$\operatorname{archeologico:}^{92}$ una corazza circolare senza decorazione centrale (tipi Numana, Paglieta o Alfedena) o una corazza liscia che non aveva la forma ad otto ma rotonda (come il tipo Mozzano).

Per quanto riguarda le stele di Penna Sant'Andrea, esse vennero portate alla luce nel novembre del 1973 in occasione di lavori agricoli sul pendio settentrionale della collina che ospita (a circa 500 metri di distanza), sulla cima, a quota 749 , il santuario di Monte Giove, nel comune di Cermignano. ${ }^{93}$ In seguito a tale ritrovamento furono effettuate nel 1974-75 due campagne di scavo che permisero di individuare, in situ, il frammento basale della stele integra (nella stessa area in cui erano già state localizzate le altre) e nove tombe a fossa in uso tra VII e IV secolo a. C. In base ai dati di scavo è molto probabile che le tre stele di Penna Sant'Andrea (fig. 16) fossero disposte in fila, forse in ordine decrescente di altezza, sul lato occidentale del tumulo (non scavato) testimoniato dalla porzione di crepidine in pietra portata alla luce nel 1974. Lo scavo non ha raggiunto la deposizione centrale del tumulo che resta ancora sepolta mentre la tomba 2, di età arcaica (che costituisce, al momento, l'unico terminus ante quem disponibile per

l'impianto della tomba centrale), e poi le sepolture 1 e 3 (databili al IV sec. a. C.) sono deposizioni posteriori all'edificazione del tumulo disposte, come di prassi, all'esterno della sua delimitazione. Le stele in pietra di Penna Sant'Andrea così come le due rinvenute nell'ottocento lungo il fosso Pantano, tra le rovine del castello di Sant'Andrea e Colle della Civita, non lontano dal villaggio di Ripattoni, nel territorio di Bellante e conservate nel Museo Archeologico di Napoli, non hanno nulla a che vedere con gli ideali eroici del gruppo Numana-Capestrano ma sembrano avere nella legge, nelle istituzioni (espresse attraverso la loro arma principale: la lingua e la scrittura) i loro punti di forza e di identità ${ }^{94}$.

\footnotetext{
92 Problema che già si pone, anche in maniera più vistosa, per l'elmo copricapo.

93 La Regina1986, pp.125-130; Guidobaldi 1995; D’ERCOLE, MARTELlONE 2005, pp. 55-124; D’ERCole, MARTELLONE 2006, pp. 55-57.

${ }^{94}$ D'ERCOLE 2007, pp. 75-79.
} 
Una ulteriore riflessione, relativamente ai due contesti di Capestrano e Penna Sant'Andrea, merita l'evento del loro 'abbattimento e seppellimento'. Tutti i frammenti di stele rinvenuti, sia occasionalmente con le arature del 1973 che con lo scavo del 1974, presentano le stesse dimensioni, circa $60 \mathrm{~cm}$ di lunghezza, come se fossero state spezzate, intenzionalmente, in modo omogeneo. Le indagini in corso potrebbero, forse, stabilire la tecnica usata per il loro abbattimento: con dei colpi di mazza (di cui non si vede traccia ad occhio nudo) o mediante l'uso di cunei. Molto più immediato ed evidente sembra il punto di abbattimento della statua del Guerriero: le caviglie che costituivano la parte di maggiore debolezza dell'intera scultura (non a caso l'espediente dei pilastri laterali). Come è ben noto alle statue di Hirschilanden e Glauberg mancano i piedi perché sono rimasti, verosimilmente, attaccati alla base in pietra considerato che, anche in questo caso, il punto d'impatto preferenziale per abbatterle erano le caviglie. A questo punto corre l'obbligo di porsi la domanda: in quale occasione e quando vennero abbattuti i gruppi scultorei di Capestrano e di Penna Sant'Andrea? Il perché costituisce forse la risposta più facile: dovevano essere venute meno le motivazioni e i significati che avevano portato alla loro creazione. È naturalmente possibile che ciascuna comunità e, quindi, in ogni necropoli, i motivi fossero specifici e legati a ragioni interne al gruppo sociale: di conseguenza tale furia 'iconoclasta' si sia scatenata in tempi, se non in modi, differenti. Esistono però dei cardini cronologici generali: le statue sostituiscono, in epoca tardo orientalizzante, all' esterno dei tumuli, sul versante di ponente, i menhir e vengono realizzate soprattutto nel corso della fase arcaica. Dopo l'epoca arcaica un fatto storico istituzionale cambia radicalmente il panorama politico tra i popoli dell'Italia centrale: la cacciata dei re, la fine dell'ordinamento monarchico e la creazione di sistemi repubblicani con cariche elettive. In Abruzzo non sappiamo con certezza se questa fondamentale svolta democratica sia avvenuta all'inizio o, più probabilmente, intorno alla metà del $\mathrm{V}$ secolo a. C. ma certo spiegherebbe perfettamente il risentimento verso il passato regime e la volontà di cancellarne ricordi, simboli, immagini e volti: una damnatio memoriae contro tutto ciò che precedeva le neonate Toutai italiche.

\title{
Analisi dei litotipi e indagini al microscopio digitale sulle sculture
}

\author{
Silvano Agostini ${ }^{95}$
}

Le analisi ad oggi eseguite sulle statue stele sono state finalizzate al riconoscimento litologico e stratigrafico dei lapidei utilizzati, al riconoscimento della giacitura dello strato (superficie e base) rispetto alla visuale frontale delle stele, alle tracce di lavorazione e di rifinitura se presente, alla individuazione delle tracce di colore/i. Per i litotipi dopo il loro riconoscimento è stata condotta una ricerca sulla cartografia e letteratura geologica disponibili, per individuare i possibili affioramenti di estrazione che potessero risultare compatibili con il sito di rinvenimento delle stele.

Le indagini archeometriche, non distruttive, sono state svolte con un microscopio digitale e specifico software capace di restituire le frequenze degli spettri di riflettanza alle osservazioni a luce normale.

\footnotetext{
95 Geologo, già Direttore della Sezione di Geologia e Paleontologia MIBACT, Docente di GeoArcheologia e di Caratterizzazione dei Materiali Lapidei presso l'Università G.d'Annunzio di ChietiPescara.
} 
Di seguito i lapidei carbonatici, costituiti tutti ad eccezione delle stele di Penna San'Andrea, da calcari sono indicati secondo la texturalclassification of carbonate rocks di Folk e Duhnam; il nome della formazione è quello noto nella letteratura geologica.

Località stele: Rapino -unsortedbiosparite-rudstone; BryozoanFormation; Middle Miocene

Località stele: Guardiagrele-sparcebiomicrite-wackestone; LithotamniumFormation; Upper Miocene

Località stele: Collelongo(packedbiomicrite - grainstone); 'Rudiste' Limestones Latium-Abruzzi shelf; UpperCretaceous

Località stele: Atessa-sparcebiomicrite- wackestone; Limestones ( $M c$ in $\mathrm{F}^{\circ} 147$ Lanciano sheet) Molise basin nappe; Early Middle Miocene

Località stele: Capestrano - sparcebiomicrite- wackestone; Bryozoan and LhitotamniumFormation; Middle Miocene

Località stele: Loreto Aprutino- sparcebiomicrite- wackestone; Bryozoan and LhitotamniumFormation; Middle Miocene

Località stele: Penna Sant'Andrea - marls with subordinates fine sands; LagaFormation (Flysch) Teramo member; Upper Miocene (post evaporitic).

In tutti i litotipi, ove presenti, si sono potuti riconoscere fossili integri o in frammenti pertinenti a gusci di molluschi bivalvi, gasteropodi, echinidi, parti di alghe e briozoi, fossili evidenti soprattutto nella superficie del dorso delle stele, la meno o affatto lavorata.

Le analisi al microscopio digitale hanno evidenziato tracce di colore, oltre a quelle note da analisi autoptica, nella stele di Collelongo, nel busto femminile di Capestrano, analogamente sono stati ottenuti spettri di riflettanza per le alterazioni (patine, concrezioni discontinue, etc.) dovute al degrado e mai o solo in parte eliminate con i restauri.

Di seguito sono riportate alcune immagini rappresentative dei risultati analitici acquisiti con questa prima fase di studio archeometrico.

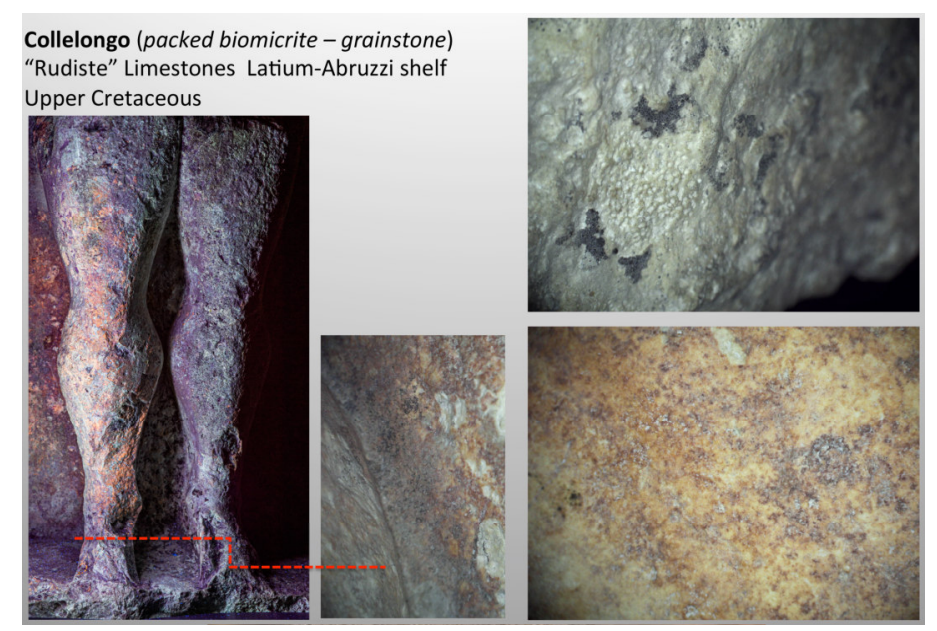

Fig.17 Stele di Collelongo: sulle gambe della stele sono evidenti tracce di colore rosso e bruno (vedi particolare in foto al centro e in basso a destra), sia sui gambali, sia sulla superficie di fondo del lapideo. Nella foto in alto a destra microfossili di alghe calcaree che caratterizzano la roccia, la patina grigiastra e resti di biodegrado (muschi). 
Frankfurter elektronische Rundschau zur Altertumskunde 40 (2020)
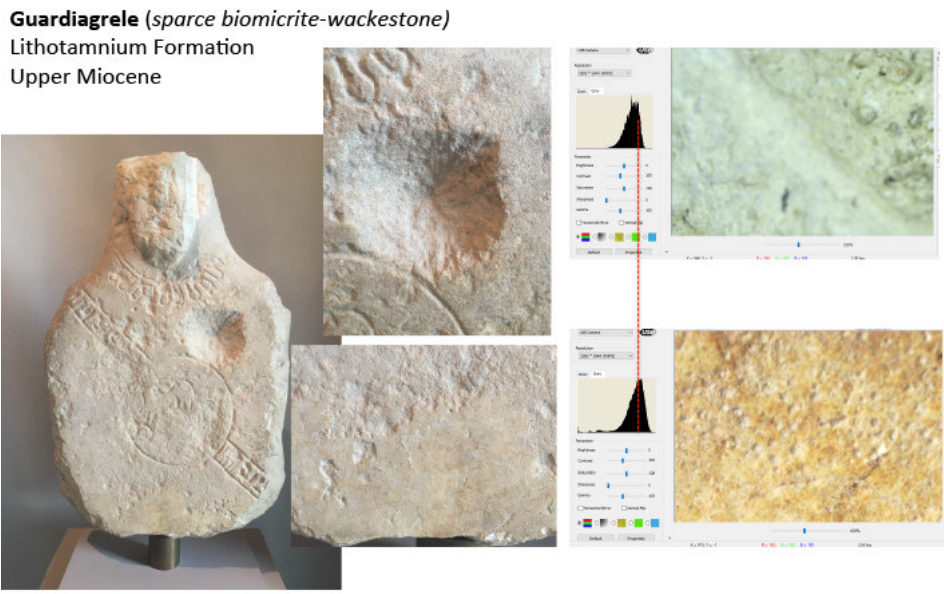

Fig. 18 Stele di Guardiagrele: Alla base della stele è ben visibile la lisciatura conservata eseguita sulla superficie dello strato di spessore medio utilizzato. A destra, in alto particolare del solco di lavorazione eseguito con scalpello, in basso numerosi fossili e microfossili. Gli spettri di riflettanza sono tipici del calcare in coerenza con quelli della figura 17
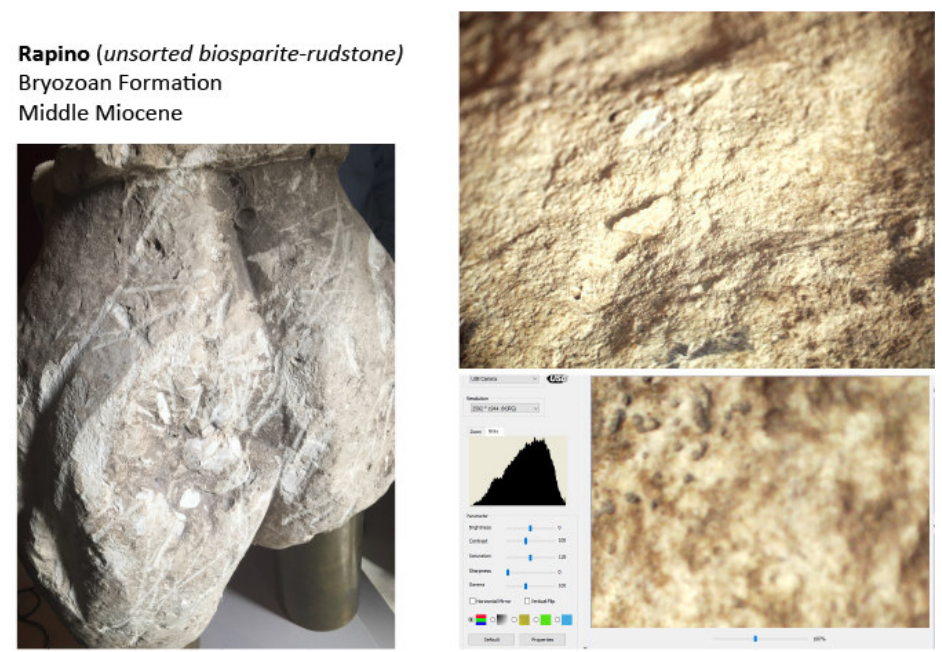

Fig. 19 Stele di Rapino: Nella lacuna del dorso è possibile riconoscere numerosi fossili e microfossili che attestano la pertinenza litobiostratigrafica del lapideo.
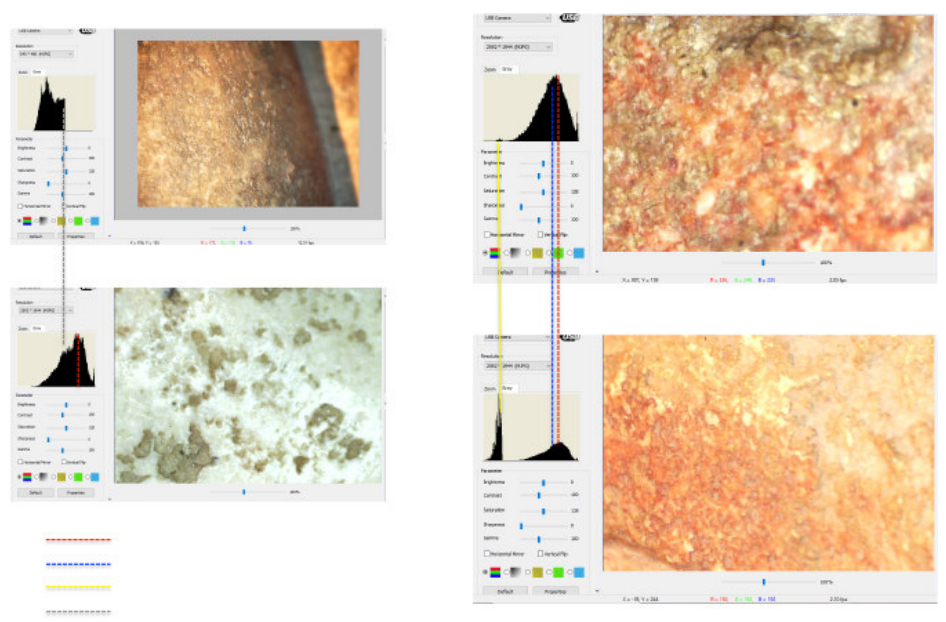

Fig. 20 Stele di Capestrano: Le figure mostrano i diversi colori usati e i relativi spettri, in basso a destra lo spettro della roccia e delle concrezioni di ossalato. 
Frankfurter elektronische Rundschau zur Altertumskunde 40 (2020)
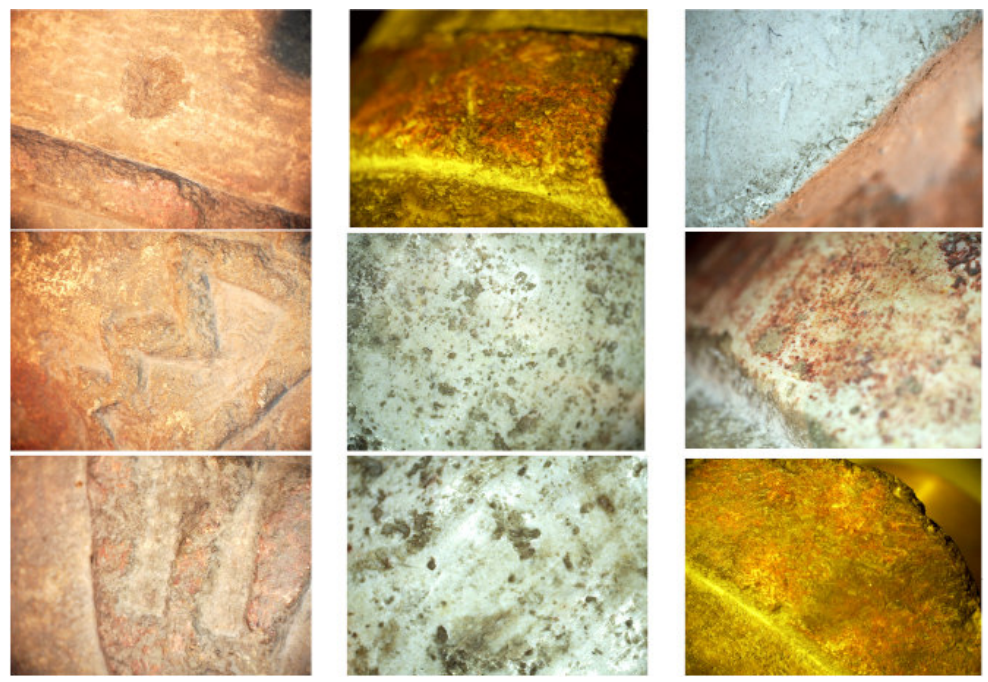

Fig. 21 Stele di Capestrano: Le figure mostrano le lavorazioni ottenute con diversi strumenti, le lisciature, e i colori usati anche, il bianco, come preparazione.
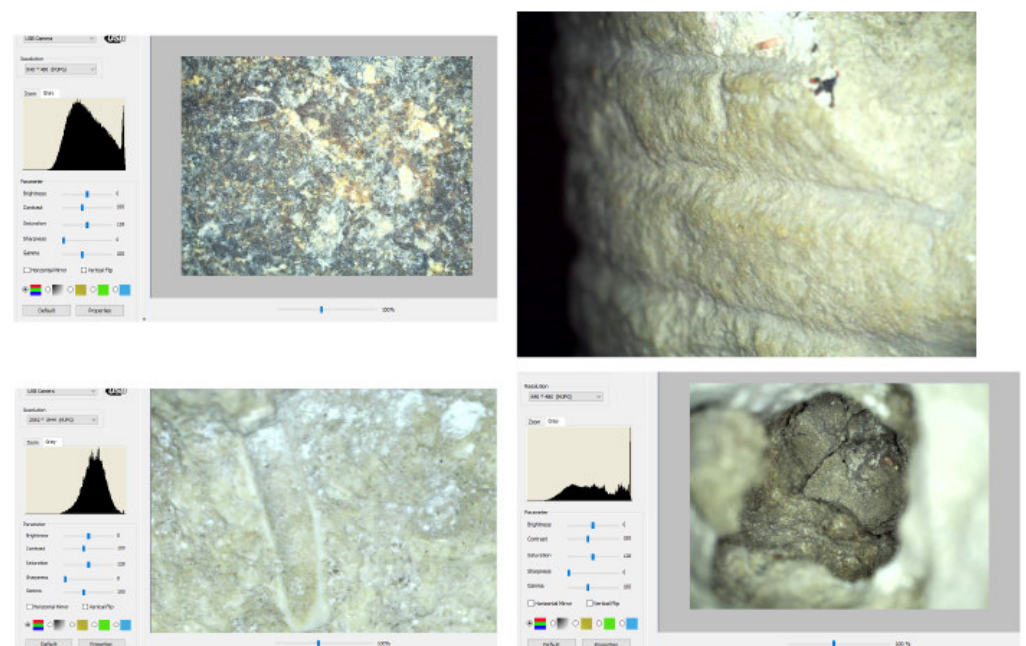

Fig. 22 Stele di Atessa: In alto a sinistra patina di alterazione e relativo spettro, in basso a sinistra particolare della lavorazione e spettro lapideo. A sinistra in alto particolare della lavorazione eseguita con identico scalpello, in basso a sinistra argilla con microfossile di Globorotalia (terreno di seppellimento) conservatasi in alcuni fori della stele. L'argilla per età è coerente con il lapideo calcareo della stele, in affioramento questo litotipo è in alternanza infatti con i calcari.

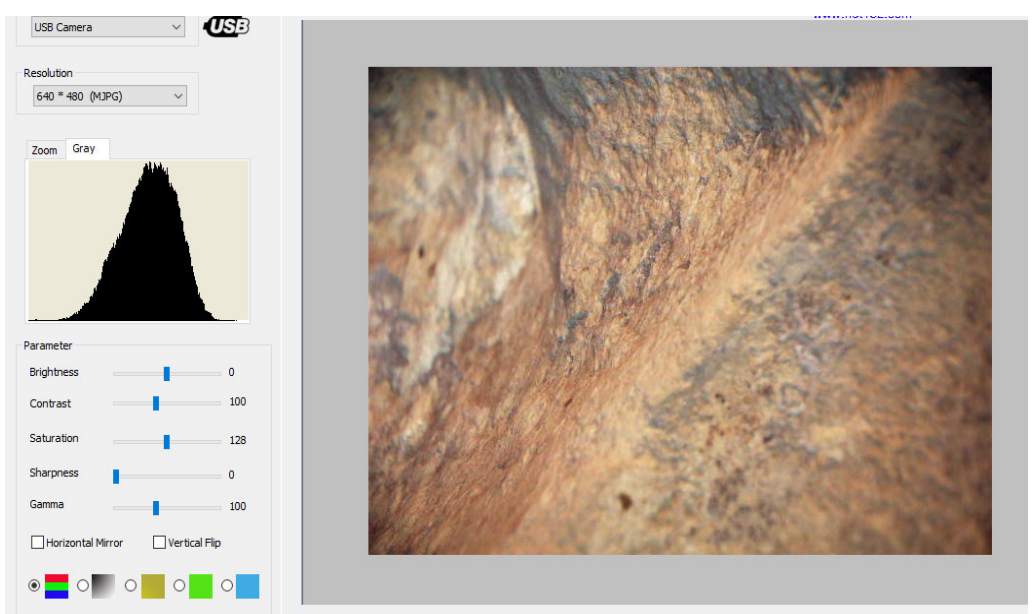

Fig. 23 Stele di Loreto Aprutino: Lo spettro della roccia, tipico del calcare, e a destra particolare della lavorazione della mascella.

http://www.fera-journal.eu 
Le indagini sul guerriero di Capestrano sono state essenzialmente una verifica e un approfondimento di studi già svolti negli anni passati (AGOSTINI 2005, pp. 12-13; AGOSTINI 2010, pp. 226-229). La statua stele è costituita da uno strato di spessore medio di calcare in facies di rampa carbonatica del Miocene medio, riferibile al Gruppo di Bolognano. Affioramenti di questi litotipi sono presenti nei rilievi in destra idrografica

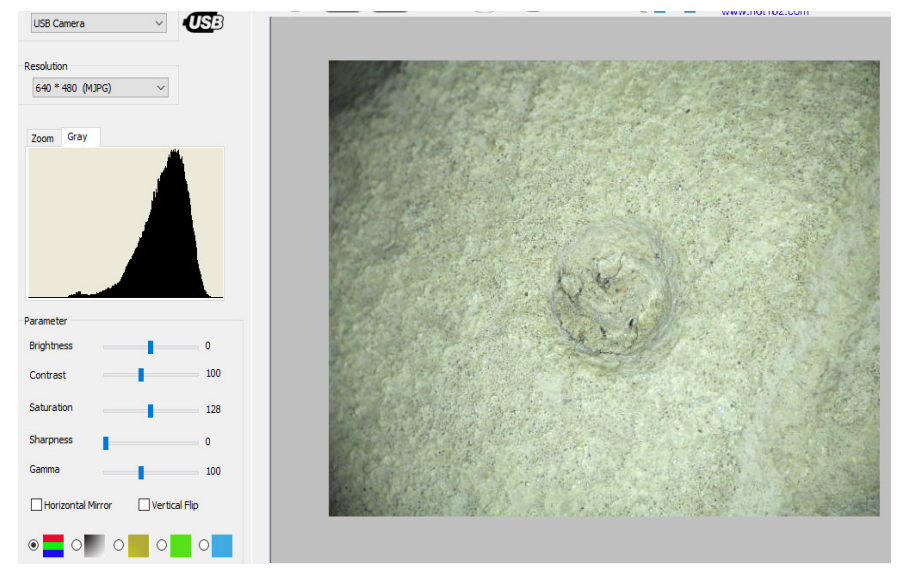

Fig. 24 Stele di Penna Sant'Andrea: Spettro della roccia, una marna calcarea con clasti costituiti da frammenti litici di rocce sedimentarie e metamorfiche, e da minerali di quarzo (prevalenti), particolare di un foro pertinente l'iscrizione.

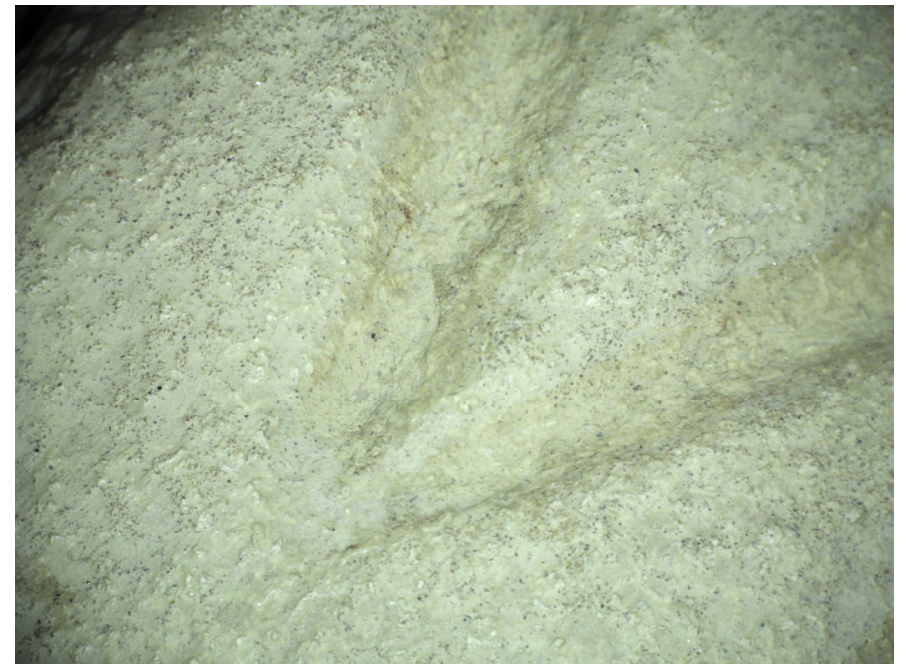

Fig. 25 Stele di Penna Sant'Andrea: Particolare della superficie del lapideo e dei solchi dell'iscrizioni a scalpello ad elevato ingrandimento.

del fiume Tirino, quindi nell'area della necropoli di Capestrano. Nei lavori precedenti la ricampionatura digitale di alcune immagini acquisite dagli archivi, anche dalla stampa estera, hanno permesso di analizzare lo stato di conservazione della parte superiore della statua poco dopo il suo ritrovamento nel 1934, in occasione della sua esposizione a Roma presso il Museo delle Terme (Fig. 26).

Le altre immagini studiate sono relative al 1936, riprese poco dopo il restauro operato sotto la guida di G. Moretti (MORETTI 1936). Nei primi documenti fotografici è evidente la presenza di numerose e invasive concrezioni di ossalato come pure di diffuse lacune piccole e medie (centimetriche), successivamente rimosse in parte le prime e stuccate le seconde, in occasione nel primo restauro. Per la stuccatura fu utilizzata, come allora era di norma, una pasta costituita da polvere di pietra, biacca e soprattutto da pece greca 
(un residuo della distillazione della resina estratta dal Pino silvestre chiamato anche Colofonia). Le tre componenti furono miscelate e amalgamate a fuoco e poi applicate a caldo. Alla statua furono aggiunti come integrazioni una cresta sul cappello e dei calzari alle gambe. Valerio Cinfarani successivamente con un altro intervento tolse queste parti ritenute arbitrariamente ricostruite dunque non veritiere e soprattutto, per i calzari, poco coerenti (Fig. 27)

Le due parti i cui era frammentata la statua furono risarcite tra loro con l'apposizione di perni in lega metallica affogati in piombo (Fig. 28).

Analoghi perni di lega metallica più sottili sono presenti anche nel cappello del guerriero. Le indagini archeometriche hanno messo in luce la tecnica di lavorazione eseguita con scalpelli a diverse punte o a punta piatta; l'accurata lisciatura ottenuta con soffice abrasivo, probabilmente ottenuto dai limi bianchi lacustri del Pleistocene inferiore presenti anch'essi nell'area di Capestrano, costituiti in prevalenza da microscopici gusci silicei di diatomee. L'alterazione della roccia è stata determinata

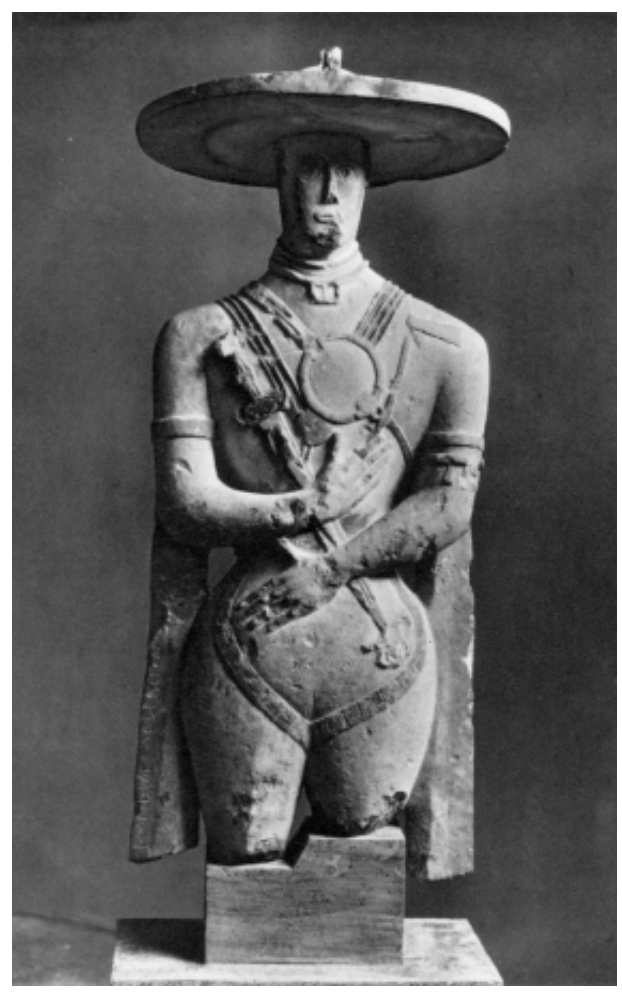

Fig. 26 La statua fu rinvenuta durante l'aratura in un suolo rimodellato dalle pratiche agricole, ma riconducibile ai tipi presenti nelle conche intermontane. $\mathrm{Si}$ tratta di un Inceptisuolo, con petrosità media, di colore bruno scuro - bruno grigio, franco sabbioso, con sostanza organica media, senza calcare attivo e alta capacità di scambio cationico. Il profilo tipo è costituito dai tre orizzonti: $\mathrm{A}>\mathrm{B}_{\mathrm{w}}>\mathrm{C}$. soprattutto dagli attacchi operati dai processi fitocarsici, mentre sono state quasi del tutto asportate con il primo restauro le concrezioni di ossalati evidenti nella documentazione fotografica, come quella ad esempio sul lato destro della bocca (Fig. 29).

Le operazioni di rimozione degli ossalati non sono state sempre indolori perché sono risultate spesso invasive compromettendo la sottostante superficie finita.

In questa nuova fase di studio le osservazioni effettuate direttamente con luce UV o tramite analisi di immagine hanno permesso di confermare con un dettaglio maggiore le tracce di lavorazione e più in generale di definire tutte le parti dove risulta conservato il colore rosso applicato (Fig. 30), le aree di degrado e degli interventi di restauro e di conservazione succedutisi nel tempo. Da ultimo è interessante anche dal punto di vista archeologico la mappatura delle velocità eseguita con indagini della tecnica degli ultrasuoni (Fig. 31). Esse evidenziano rotture nette, simmetriche a carico della statua stele avvenute a seguito dell'applicazione (urto) di forze impulsive generate da un evento (caduta) forse non naturale. 


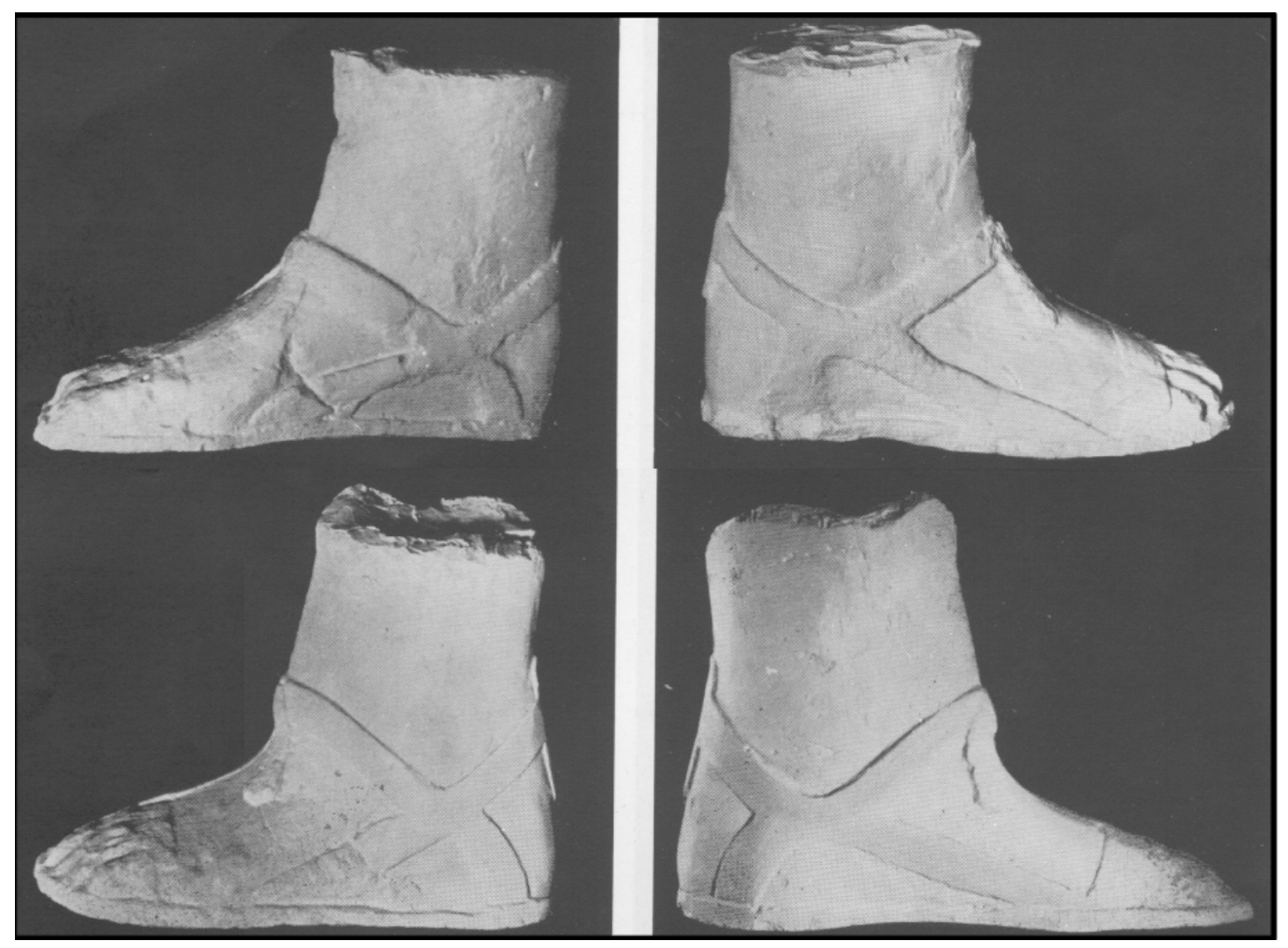

Fig. 27 Nel secondo restauro, operato sotto la guida di V. Cianfarani, (CIANFARANI 1968 pp. 519) alla statua del guerriero sono state eliminate le integrazioni dei calzari, con $i$ "fantasiosi fiocchetti in contrasto con la rude severità globale del monumento".
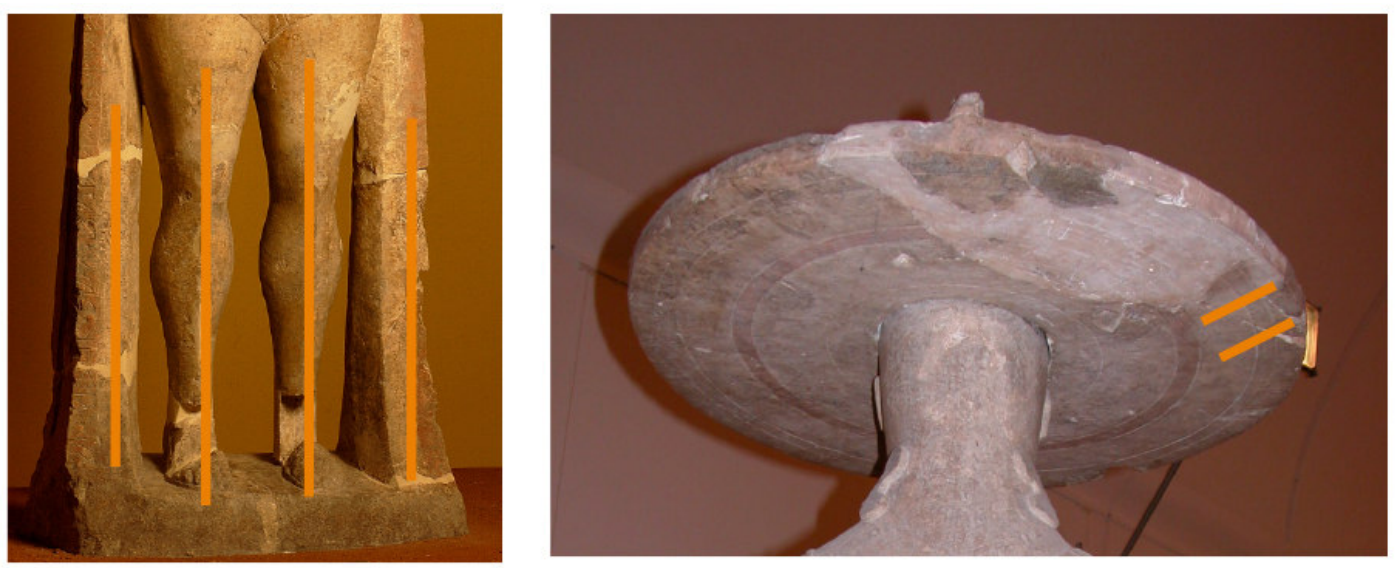

Fig. 28 I perni inseriti nelle gambe, nei fianchi e nel copricapo con il primo intervento di restauro eseguito sotto la guida di G. Moretti. I perni sono stati definiti con analisi non invasive di tipo "Eddy Current" condotte dalla allora Soprintendenza ai Beni Archeolgici dell'Abruzzo in collaborazione con l'ENEA. I perni risultano costituiti da una lega metallica (tipo ottone) "affogata in piombo". 


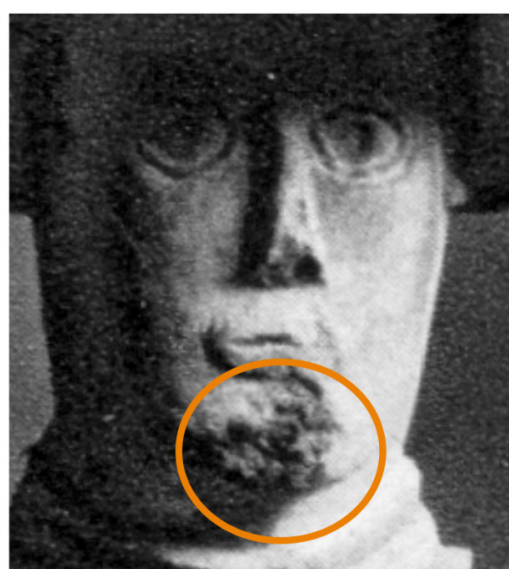

Fig. 29 Particolare della concrezione costituita da ossalati di calcio, estesa a coprire parte della bocca fino al mento. I batteri carbonatogeni che vivono nel suolo hanno determinato nel tempo sulla statua sia concrezioni di ossalati di calcio che una patina protettiva. Le alghe endolitiche hanno invece determinato diffuse microcavità di corrosione (fitocarsismo).
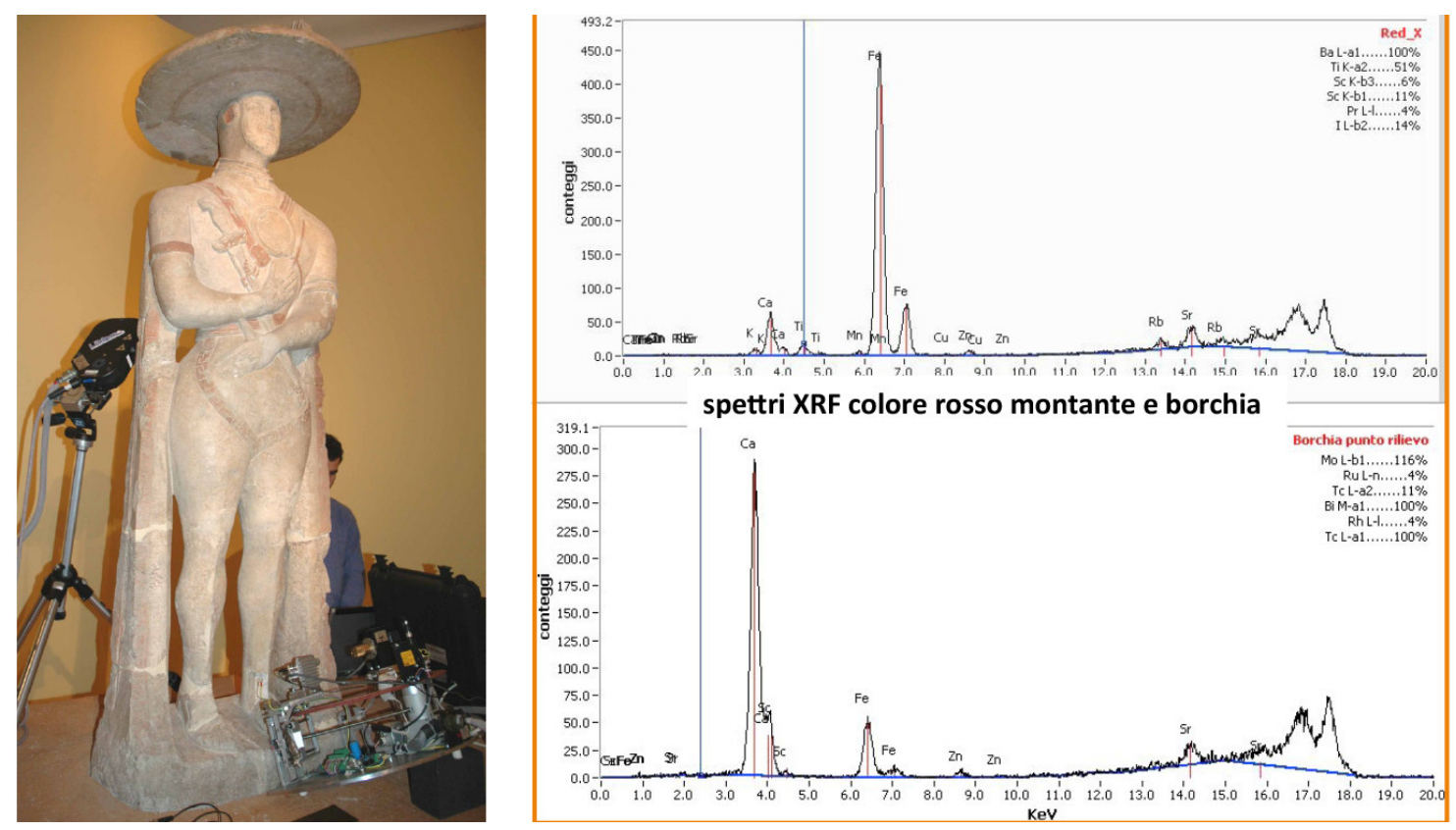

Fig. 30 Analisi condotta mediante strumentazioni portatili XRD e XRF per la caratterizzazione del colore e dei prodotti superficiali di degrado.
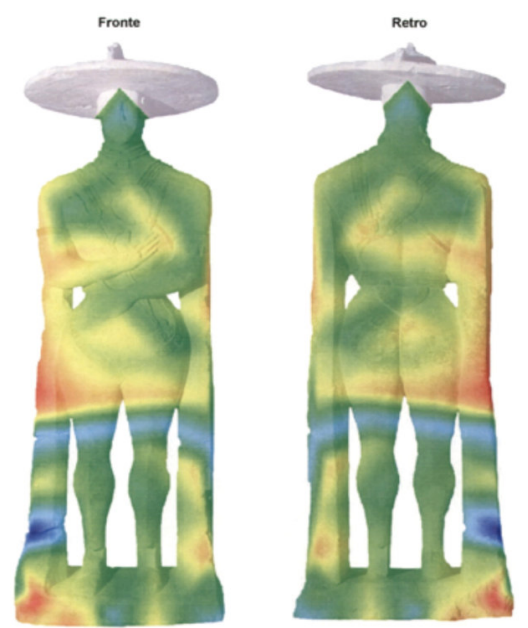

Fig. 31 I risultati espressi come mappa a colori della velocità ultrasonica mettono in evidenza in relazione allo spessore della pietra, che le fratture del monoblocco scolpito della statua sono nette e non risultano pervasive delle parti giustapposte (perdita di coesione e densità). Questo lascia supporre che il "trauma" meccanico sia stato impulsivo e riconducibile a un unico evento: una causa antropica più che un crollo naturale. In questo quadro la rottura del basamento risulterebbe anomala suggerendo almeno per questo elemento una rottura avvenuta in una fase successiva. 


\title{
Tracce di lavorazione e tool-marks
}

\author{
Eugenio Di Valerio ${ }^{96}$
}

Delle numerose discipline di cui ci si avvale oggi nell'analisi di un monumento antico, quella archeologica è arrivata a comprendere numerosi approcci che travalicano il solo esame autoptico del manufatto in sé e si occupano di tutta quella serie di processi

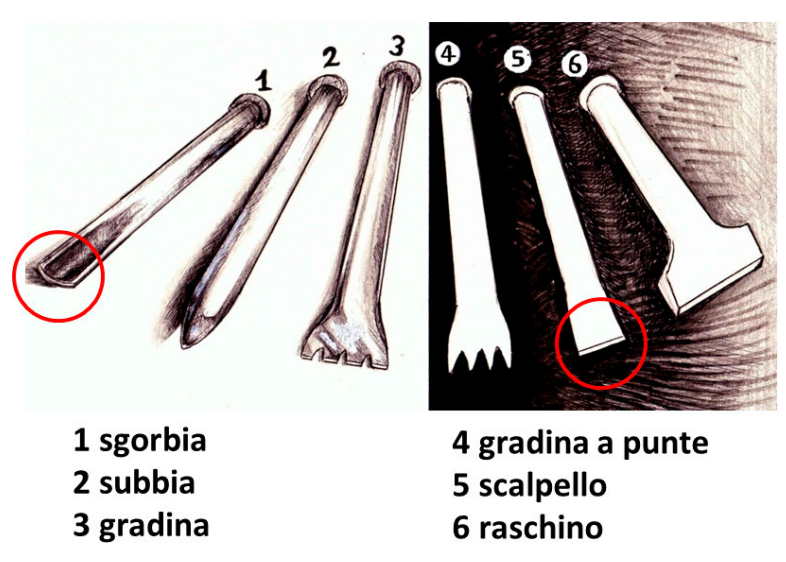

Fig. 32 Strumenti a percussione indiretta per la lavorazione della pietra. attorno alle fasi di lavorazione. Ogni materiale subisce un processo più o meno lungo di trasformazione che porta la materia prima a divenire idonea all'utilizzo che se ne vuole fare. L'importante punto di vista offerto dall' archeometria fornisce spesso le basi per un'analisi sempre più puntuale dei manufatti antichi; una lettura che tenga conto delle numerose variabili che incorrono nella realizzazione di un determinato oggetto getta un ponte tra la storia descritta e quei reali processi di produzione che interessarono la cultura materiale antica. Nelle tracce lasciate dagli utensili è possibile distinguere quelli lasciati da strumenti a percussione diretta in quanto caratterizzati da tratti netti e brevi e spesso molto profondi; al contrario gli strumenti a percussione indiretta, retti da una delle due mani e colpiti da un percussore dall'altra, lasciano tracce di tipo continuo, lunghe e normalmente meno profonde rispetto alle prime. Da alcuni indici di regolarità delle tracce strumentali è possibile risalire all'esperienza dell'artigiano/cavatore/scultore. Questi indici sono: 1'uniformità del tratto, la profondità delle tracce, la regolarità nell'andamento rettilineo, i rapporti di parallelismo/perpendicolarità tra le diverse tracce, la distanza costante tra i tratti e il mantenimento dell'angolo d'impatto. Queste tracce ci permettono anche di ipotizzare la presenza di diverse maestranze con diversi livelli di manualità che compartecipano all'azione di lavoro, sia essa di cavatura o lavorazione successiva. Irregolarità nell'uso della gradina portano ad avvallamenti dovuti alla variazione dell'angolo orizzontale dell'utensile durante la lavorazione. Tra le operazioni di regolarizzazione e spianamento delle superfici talvolta ve ne sono alcune che sono già un grado di finitura; in determinati contesti, soprattutto relativi a materiale edile, le superfici vengono volutamente lasciate scabre mediante bocciardatura o spuntatura.

La lavorazione della superficie realizzata con bocciarda è riconoscibile grazie alla presenza di patterns dovuti alla forma a maglia regolare dello strumento; lo stesso risultato si può ottenere con la subbia ma in questo caso non è riscontrabile la presenza di uno schema regolare ricorrente. La spuntatura realizzata a subbia è riconoscibile grazie ai brevi segni lasciati dallo strumento che rientrano nell'orine del centimetro; talvolta, quando le maestranze sono ben specializzate, questi segni risultano rari in

\footnotetext{
${ }^{96}$ Archeologo ed esperto di tracce di cavatura e scolpitura, borsista co-titolare dell'insegnamento di Archeologia delle Colonie Greche, Università G.d'Annunzio di Chieti-Pescara.
} 
quanto ogni colpo oblitera il segno lasciato dal precedente lasciando solo la rugosità della frattura.

Per quanto riguarda l'esame dei tool marks un caso particolarmente interessante è rappresentato dal Torso di Pallano, dove è possibile riscontrare la presenza di scalpelli piatti unitamente a larghe sgorbie nonché un ulteriore strumento a punta che modifica a posteriori la superficie finita del manufatto.

Lo scalpello piatto è stato utilizzato in tre modi distinti:

1) con una inclinazione di circa $40^{\circ}$ sul piano verticale e $40^{\circ}$ su quello orizzontale per spianare le superfici (ad es. sulle facce frontali dei pilastrini laterali);

2) perpendicolare al piano orizzontale e inclinato con un angolo variabile, a seconda della curvatura della superficie, nella modellazione dei volumi e nella realizzazione dei sottosquadri (ad es. sulle braccia);

3) nell'incisione dei dettagli (cinturone, dita, piegatura del gomito) lo scalpello viene utilizzato nel primo modo ma con due passaggi necessari alla realizzazione dei solchi con profilo a $\mathrm{V}$.

Sulle facce laterali dei pilastrini si nota l'impiego di una sgorbia molto larga e con una curvatura minima utilizzata probabilmente nella fase di sgrossatura del blocco. La superficie, in cattivo stato di conservazione, mostra una patina discontinua frutto probabilmente di una rifinitura grossolana; si trattava probabilmente di una lisciatura realizzata per abrasione mediante pomici o smerigli a secco che non penetrano nei solchi maggiormente incisi e nei sottosquadri.

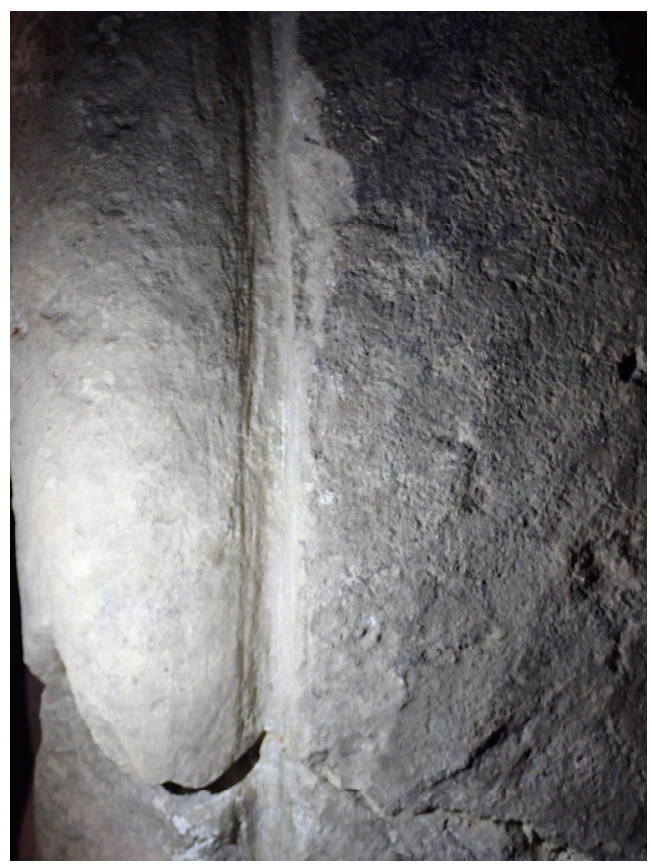

Fig. 33 Tracce di impiego di una sgorbia molto larga e con una curvatura minima.
Tracce recenti di strumenti grossolani interrompono la patina da alterazione del manufatto sulla parte alta della schiena e del trapezio; si tratta di profondi solchi compatibili con le tracce lasciate su altri manufatti da benne, picconi o vomeri di aratro.

Sul petto e torace sono presenti invece tracce, sempre recenti, di una subbia o una piccozza a punta che insistono nell'area della lacuna centrale. L'ipotesi quindi di una possibile panoplia incisa risulta ascrivibile a interventi moderni.

Un'ultima serie di segni, in parte certamente recenti, è costituita da incisioni superficiali "a graffio" realizzate senza l'ausilio di un percussore e presenti su tutta la superficie frontale dalle spalle al ventre.

Tra queste ultime due sembrerebbero, ad una prima analisi, più antiche delle altre: un breve tratto di circa $12 \mathrm{~cm}$ con andamento diagonale sull'alto ventre e due tratti paralleli sotto il costato sinistro. 


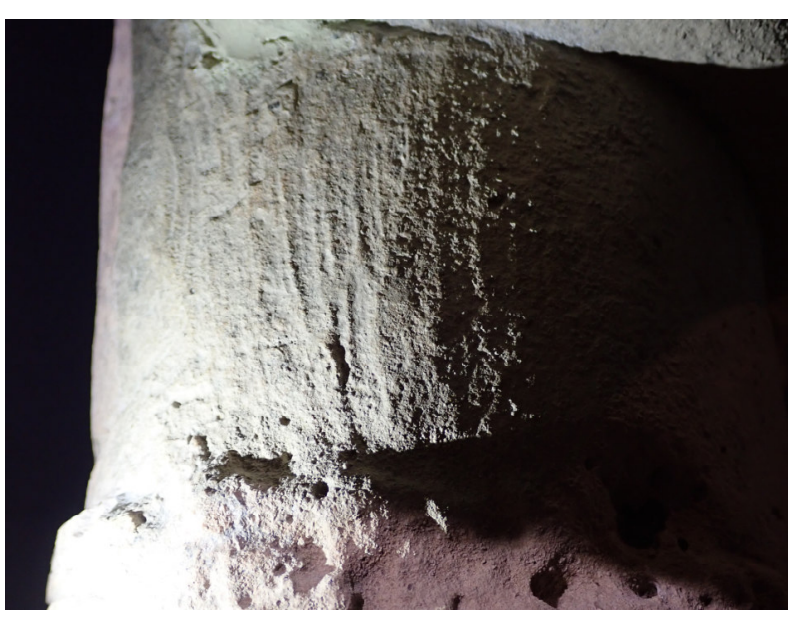

Fig. 34 Tracce di impiego di uno scalpello piatto.
L'utilizzo di questo tipo di strumenti, della sgorbia in particolare, lascia intendere una buona conoscenza da parte dell'artigiano delle tecniche scultoree normalmente impiegate sul legno. Questo aspetto della più antica statuaria italica purtroppo è supportato da pochissimi rinvenimenti 97 , tuttavia le prime forme del sacro, al pari degli xoana, degli àgalma e/o degli acroliti di ambiente greco $^{98}$, dei quali sappiamo da Pausania essere in alcuni casi sopravvissuti fino al II sec. d.C.,

erano verosimilmente lignee anche in ambiente italico. D'altronde anche a Roma non mancavano esemplari in legno fra le più antiche statue di culto, per quanto essi siano affiancati da un uso più cospicuo della terracotta, secondo la tradizione etrusca; oltre al simulacro di Veiove, erano lignee le antiche statue di Diana Aventina e della Fortuna Muliebris; peraltro nello stesso mondo etrusco è attestata l'esistenza di una statua di Iuppiter ottenuta da un tralcio di vite a Populonia (Plin.N.h., XIV,9). Questa stessa tipologia di statue lignee non era sconosciuta neanche ai celti dell'Europa centro occidentale come testimonia la testa rinvenuta nel 1973 a Soulac-sur-mer ${ }^{99}$.

\section{I protocolli fotografici e il 3D fotogrammetrico}

$$
\text { Rocco D’Errico }{ }^{100}
$$

La moderna tecnologia di acquisizione ed elaborazione digitale delle immagini ha messo a disposizione dell'archeologia nuove possibilità di analisi e remote sensing. Infatti la fotografia digitale può essere usata non solo per realizzare in maniera più semplice ed efficace le tradizionali foto di documentazione dei reperti, ma offre nuove interessanti possibilità di analisi come la fotogrammetria 3D (SMF). Inoltre la tecnologia agevola notevolmente la possibilità di trasferire le immagini, rendendone facile ed immediata la condivisione tra i membri dell'equipe, nonché di pianificarne una fruizione più diretta.

Oltre all'opera nella sua interezza, l'oggetto privilegiato del nostro protocollo di analisi è la superficie della scultura che viene scandagliata in maniera approfondita con diverse tecniche di acquisizione delle immagini, cercando di rivelare: i segni delle

\footnotetext{
${ }^{97}$ Tra i più rappresentativi vale la pena di ricordare lo xoanon proveniente dal santuario di Mefite in Valle d'Ansanto, rinvenuto negli anni '50 del Novecento da Giovanni Oscar Onorato, insieme ad altre quattordici figurine lignee più piccole e attualmente esposto nel Museo Irpino di Avellino (inv. 1499). ONORATO 1960, pp. 12, 25-27, 32-35; RAININI, BOTTINI, ISNENGHI COLAZZO 1976, pp. 374-382.

${ }^{98}$ In generale vedi DONOHUE 1987.

${ }^{99}$ BONEFANT et al. 1998 , pp. 66-67.

${ }^{100}$ Fotografo e Archeologo, esperto di Fotografia digitale e 3D modeling applicati ai Beni Culturali. Borsista CAAM-DisPuTer, Università G.d'Annunzio di Chieti-Pescara.
} 
tecniche di lavorazione, le differenze di patina, tracce di pittura, tracce di restauro, il tipo di materiale litico ecc.

Le immagini digitali quindi non solo forniscono dati preziosi ma possono suggerire nuovi spunti di ricerca e ove effettuare nuove analisi con altre tecniche di remote sensing.

Abbiamo tenuto in considerazione il rapporto tra $\mathrm{i}$ costi sostenuti e i risultati ottenuti, e abbiamo scelto tecniche di acquisizione delle immagini tutto sommato ' $l o w$ cost', che per quanto sofisticate sono comunque economicamente accessibili e non richiedono tempi assai lunghi di produzione. A seguire l'elenco dei protocolli fotografici con una breve descrizione.

- Foto ad Alta risoluzione - Le foto ad alta risoluzione vengono effettuate con macchine ad alto numero di megapixel, ovvero dai $36 \mathrm{mpx}$ in su, oppure mettendo insieme con la tecnica del photo stitching più scatti effettuati con macchina da almeno $24 \mathrm{mpx}$. Questa tecnica viene eseguita montando la fotocamera con un obiettivo a focale normale o piccolo tele, ovvero da $50 \mathrm{~mm}$ a $105 \mathrm{~mm}$, su una testa panoramica. Una volta calibrata la posizione della macchina fotografica con la ricerca del giusto punto nodale, vengono effettuati almeno quattro scatti di parti della statua, che poi vengono uniti con un software di photo-stitching, nel nostro caso PT.Gui. Il risultato finale è un'immagine ad altissima risoluzione che consente di osservare anche i minimi dettagli della scultura come se si fosse davanti ad essa. La facilità con cui si possono diffondere le immagini digitali ad alta risoluzione che nonostante la dimensione elevata del file possono essere anche caricate su siti web, consente di coinvolgere nello studio delle sculture un maggior numero di studiosi, anche chi non può recarsi nel luogo in cui sono conservate. L'accuratezza dei dati e la facilità di condivisione sono $\mathrm{i}$ punti di forza di questo tipo di tecnica. Le foto sono state realizzate con luce artificiale utilizzando schemi di illuminazione appositamente pensati per valorizzare le forme e le caratteristiche peculiari delle sculture. A seconda del tipo di soggetto abbiamo quindi utilizzato schemi con tre o più punti luce composti da una luce principale di solito a 45 gradi di fronte al soggetto in posizione leggermente elevata e diffusa o da un softbox o da un ombrello, da una luce di schiarita in posizione opposta alla principale, da una luce d'effetto posta dietro al soggetto in posizione elevata per dar rilievo alla silhouette della scultura.

- Fluorescenza Ultravioletta UVF - La fotografia in fluorescenza UV (Fig.35) sfrutta la capacità della luce ultravioletta ${ }^{101}$ di stimolare la fluorescenza di alcuni materiali che a seconda della loro natura risponderanno a questa irradiazione generando una luce generalmente compresa nello spettro visibile ${ }^{102}$. Per questo tipo di fotografia oltre ad una normale macchina digitale, occorrono un ambiente oscurato e una fonte di luce UVA o ad onde lunghe UV, ovvero nella banda dello spettro luminoso compresa tra i 315 e i 380 nanometri, di solito con picchi di luminosità intorno ai $365 \mathrm{~nm}^{103}$. Si possono usare luci di vario tipo appositamente create per questo tipo di utilizzi come i neon con filtro di Wood a luce nera, oppure si possono montare dei filtri UV pass sui flash fotografici per avere più potenza, bisogna però stare attenti alla tendenza di neon e filtri

\footnotetext{
${ }^{101}$ IANNACONE 2014, pp. 45-56.

102 BRINKMANN 2004 pp. 35-36.

${ }^{103}$ WILLIAMS, G. WILLIAMS 2005PP 23-28.
} 
economici di lasciar passare anche della luce nello spettro visibile e dell'infrarosso vicino. In questo caso si useranno filtri UV/IR block sulla fotocamera. Nel nostro caso abbiamo usato dei neon Philips TL-D 18W BLB Blacklight Blue e messo un filtro B+W tipo E 022 Giallo Medio per attenuare la luce visibile blu.

- Infrarosso riflesso e fluorescenza IR indotta da luce visibile (VIL) - La fotografia ad infrarosso riflesso prevede l'utilizzo di una macchina fotografica appositamente modificata, con la sostituzione del filtro che blocca la luce infrarossa che si trova su tutte le fotocamere digitali. Sull'obiettivo viene utilizzato un filtro nero B+W 093 IR che blocca la luce dello spettro luminoso visibile facendo passare solo la luce infrarossa dai $780 \mathrm{~nm}$ in su. L'illuminazione può essere fornita da diverse tipologie di luci, noi abbiamo usato luci ad incandescenza e luci flash. La fotografia all'infrarosso mette in evidenza particolari del rilievo che magari sono coperti da patine, come nel caso del motivo decorativo della dama di Capestrano che nella foto IR è perfettamente visibile. La fluorescenza IR indotta da luce visibile (VIL) ${ }^{104}$ viene effettuata con la stessa procedura ma vengono utilizzate delle fonti di luce filtrate per bloccare la luce infrarossa in modo che l'unica luce IR che raggiunge il sensore sia quella generata dalla fluorescenza ${ }^{105}$. Con questa tecnica possono essere individuati alcuni pigmenti antichi come il blu egizio che presenta questo fenomeno di riemissione.

- Ricostruzione Modelli 3D con tecnica SMF - La tecnica Structure From Motion o meglio conosciuta come fotogrammetria digitale, ci consente di creare un modello 3D completo di mesh e texture da una serie di fotografie. Questa tecnica nasce per la scansione di grandi edifici e grosse superfici, però negli ultimi anni la sua evoluzione l'ha portata ad adattarsi anche alla scansione di piccoli oggetti. Il punto di forza di questo tipo di tecnica è proprio la possibilità creare dei modelli 3D con delle texture fedeli all'originale e assai dettagliate. Inoltre i modelli prodotti possono essere facilmente condivisi su piattaforme tipo Sketchfab ed usati sia per lo studio e sia per la valorizzazione dei reperti. Per la realizzazione dei modelli abbiamo usato una macchina fotografica digitale da almeno $24 \mathrm{mpx}$ con l'ausilio di luci led che illuminavano il soggetto in maniera uniforme descrivendo i rilievi ma mantenendo comunque basso il contrasto luminoso. Abbiamo effettuato gli scatti in formato Raw e dopo aver sviluppato le foto in formato jpg con il software Adobe Lightroom, abbiamo processato le immagini con il software di elaborazione fotogrammetrica $3 D F$ Zephyr. La fase più delicata è stata la creazione di maschere per isolare il soggetto dallo sfondo prima di iniziare la procedura di costruzione della mesh, Zephyr mette a disposizione per questa operazione un interessante strumento: il programma Masquerade che consente di creare maschere in maniera assai intuitiva e rapida. Il modello $3 \mathrm{D}$ è stato esportato in formato obj e caricato su un account plus della piattaforma Sketchfab che fornisce un visualizzatore assai efficiente per la condivisione nel web del modello.

- Foto particolari superficie a luce radente o incidente - Le fotografie ravvicinate di particolari delle sculture associate ad un'illuminazione radente consentono di riconoscere le tracce degli strumenti di lavorazione e di individuare patine e tracce

\footnotetext{
${ }^{104}$ IANNACCONE 2014, pp. 56-57.

105 VERRI, 2009.
} 


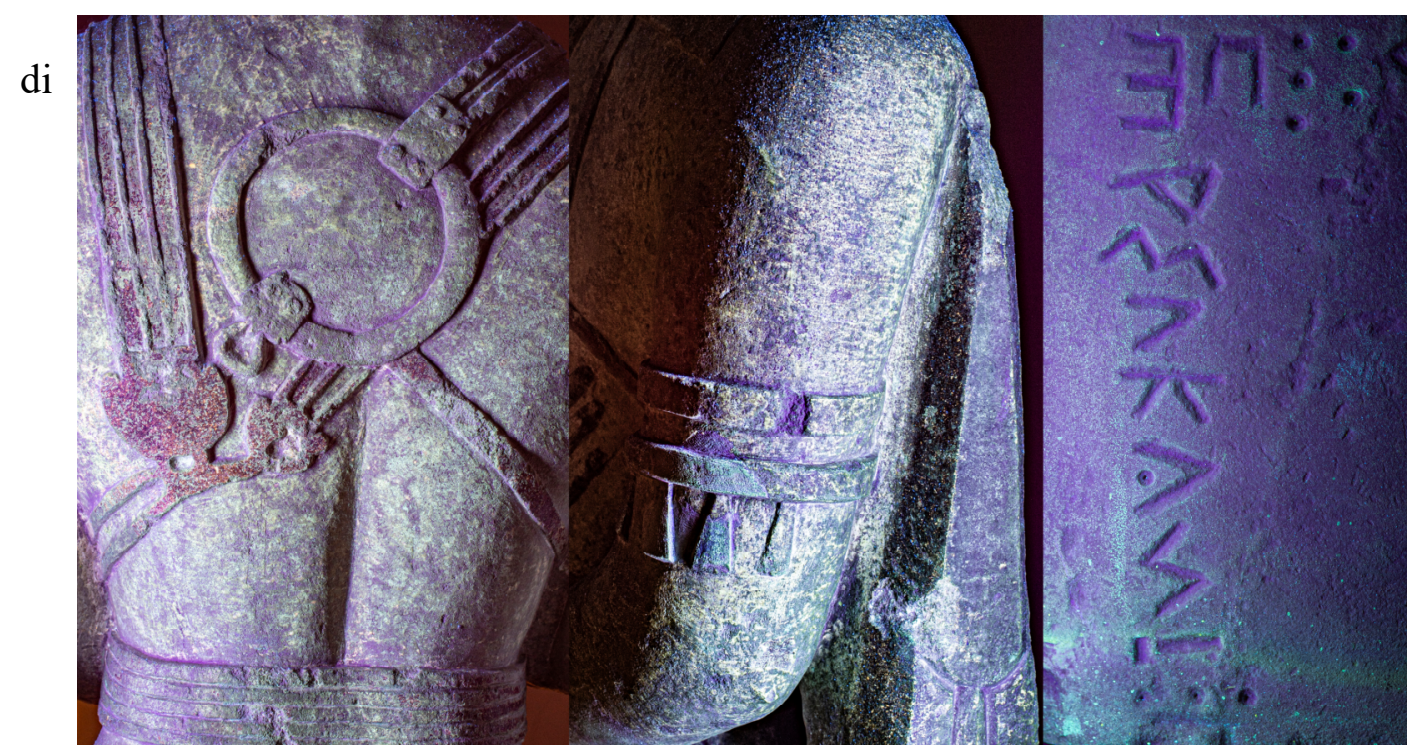

Fig. 35 Esempi di fotografie con la tecnica della fluorescenza Ultravioletta, del Guerriero di Capestrano e della Stele di Penna sant'Andrea.

pittura $^{106}$. La luce utilizzata deve essere diretta e dura, la sua inclinazione può essere variata da uno scatto all'altro per mettere in risalto a seconda della morfologia della superficie tutti i rilievi e i segni di lavorazione. Il contrasto luminoso deve essere assai duro per enfatizzare la trama della superficie ma volendo si può utilizzare una piccola luce di schiarita per non rendere le ombre troppo profonde. Per la realizzazione degli scatti sono state utilizzate ottiche macro di focale $50 \mathrm{~mm}$ e 105 $\mathrm{mm}$ e luci led fotografiche con riflettore munito di lente fresnel. Sono state effettuate delle foto a luce incidente utilizzando il flash anulare che produce un fascio di luce perfettamente parallelo all'asse di ripresa. Questo tipo di luce privo di ombre descrive la superficie scultorea sfruttando le caratteristiche di riflettenza ed enfatizzando le differenze cromatiche (Fig. 36).
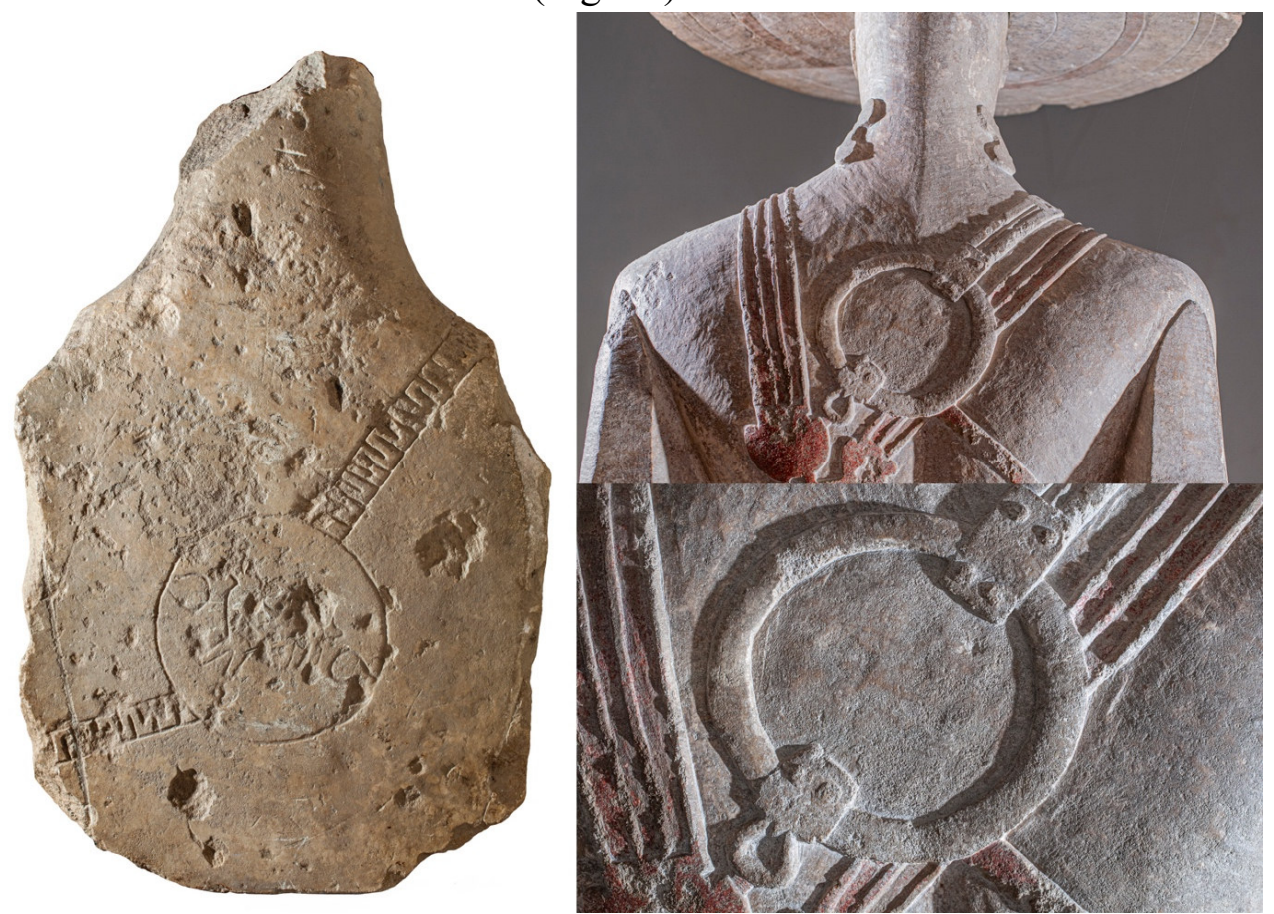

Fig. 36 Esempi di foto in luce radente della Stele di Guardiagrele e del Guerriero di Capestrano.

${ }^{106}$ V. BRINKMANN 2004 p. 35. 


\title{
Imaging iperspettrale e remote-sensing: dall'applicazione alla pittura antica a quella sulla scultura
}

\author{
Gloria Adinolfi e Rodolfo Carmagnola ${ }^{107}$
}

Da circa 20 anni un gruppo di studio interdisciplinare coordinato dalla compianta Maria Cataldi cui partecipano G. Adinolfi, R. Carmagnola, L. Marras, M. Masseti, V. Palleschi, con il supporto del CNR di Pisa sta conducendo una serie di indagini mirate sulla pittura antica anche attraverso l'utilizzo delle tecniche iperspettrali adattate alla specificità della ricerca. In questo modo è stato definito un protocollo e predisposto un software di elaborazione per consentire una modulazione progressiva in funzione dei contesti diversi ${ }^{108}$. Naturalmente i protocolli sono stati calibrati sulle necessità derivanti dalle indagini sulla pittura antica e quindi sull'analisi di una superficie piatta nella quale le variazioni cromatiche e materiche sono per lo più bidimensionali. Attualmente è invece in fase di studio la verifica di una modalità di adattamento nel passaggio dalla superficie piatta a quella tridimensionale della scultura che comporta analisi aggiuntive sulle diverse tipologie del materiale di supporto e problematiche spesso differenti, condizionate da una acquisizione che non può essere simultaneamente frontale in tutta la superficie indagata e che comporta una progettazione diversa degli illuminanti e della loro collocazione durante la ripresa.

L'acquisizione è effettuata da camera CCD monocromatica ad alta sensibilità e bassissimo rumore efficace nel recuperare livelli di segnale particolarmente deboli a causa della scarsa presenza del materiale, a volte solo in traccia. Sono stati predisposti una serie di illuminanti realizzati appositamente con LED con particolare purezza cromatica, potenza e uniformità di emissione, sia per i segnali di riflettanza dall'UV al vicino infrarosso, sia per stimolare la fotoluminescenza nel visibile (fluorescenza UV) e nell'IR (luminescenze indotte).

Il set di acquisizione è normalmente composto da circa 30 immagini monocromatiche a bande selezionate nel caso di immagine spettrale approfondita, che coprono lo spettro compreso tra i 350 e $1125 \mathrm{~nm}$ lunghezza d'onda, con l'utilizzo di filtri interferenziali ad alta trasmittanza e banda passante delimitata a coprire l'intero spettro identificato.

Alla fase di acquisizione segue una prima fase di post-processing con la predisposizione della immagini all'analisi statistica attraverso algoritmi appartenenti alla classe detta BSS (Blind Source Separation) ${ }^{109}$, selezionati ed adattati in una specifica "costruzione" dal CNR di Pisa, in modo tale da poter essere implementato con funzioni nuove in caso di diverse esigenze.

L'algortimo agisce su un insieme di immagini combinando linearmente le diverse riprese, dal momento che le diverse informazioni sono distribuite all'interno dell'intero set e raramente all'interno di una singola immagine e quindi istituisce una serie di collegamenti tra gli elementi relazionabili sulla base dell'analisi di componenti indipendenti o sulla correlazione di canali spettrali liberandoli dai pattern di interferenza ${ }^{110}$.

\footnotetext{
${ }^{107}$ Archeologi di Pegaso srl.

108 ADINOLFI ET ALII 2019.

109 TONAZZINI, GeraCE, MARTINELli 2010.

110 Salerno et ali 2014: Salerno E., TonazziniA., Grifoni E., Lorenzetti G., Legnaioli S., LEZZERINI, M., PALLESCHI V. 2014, pp. 22-27.
} 
Si viene così a creare un set di immagine aggiuntive sulle quali viene effettuata una ulteriore fase di post-processing che provvede alla comparazione dei risultati del set elaborato dall'algoritmo con le singole riprese del set multispettrale e con il visibile, relazionandole alle altre analisi archeometriche e costruendo il presupposto per lo studio archeologico di tipo iconografico o per le analisi delle anomalie di tipo conservativo.

\title{
Laser scanning per le future analisi della riflettanza
}

\author{
Donato Palumbo ${ }^{111}$
}

La diffusione preponderante dell'utilizzo di nuove tecnologie e l'intercomunicazione tra le stesse, stabiliscono un profondo legame, ormai inscindibile, tra innovazione tecnologica e studi archeologici. Mentre è indubbio il reciproco conforto, appaiono confusi i limiti e le competenze dei singoli ambiti di ricerca. La tecnologia laser scanning, ormai prassi negli approfondimenti d'indagine archeologiche, viene spesso vista come il placebo per tutti i mali, fuorviati dalla spettacolarità e dal realismo dei modelli tridimensionali, risultati pregevoli, di grande impatto, che si limitano spesso a una mera dimostrazione 'muscolare' se non confortati da chi sa leggere, interpretare e ben utilizzare le innumerevoli possibilità di output. Legame imprescindibile, tra le nuove tecnologie e l'archeologia, che non deve implicare solo la fase finale di questi processi tecnologici, l'unione deve partire a monte, sin dalle prime fasi di acquisizione dei dati, dove è indispensabile la capacità di "saper vedere" e razionalizzare il rilievo. Mannoni mette in evidenza che: "non si può fare una buona archeologia, e cioè dei buoni ragionamenti critici, senza una buona archeografia, ovvero una buona descrizione oggettiva dei dati di partenza, perché non si può avere oggettivamente in

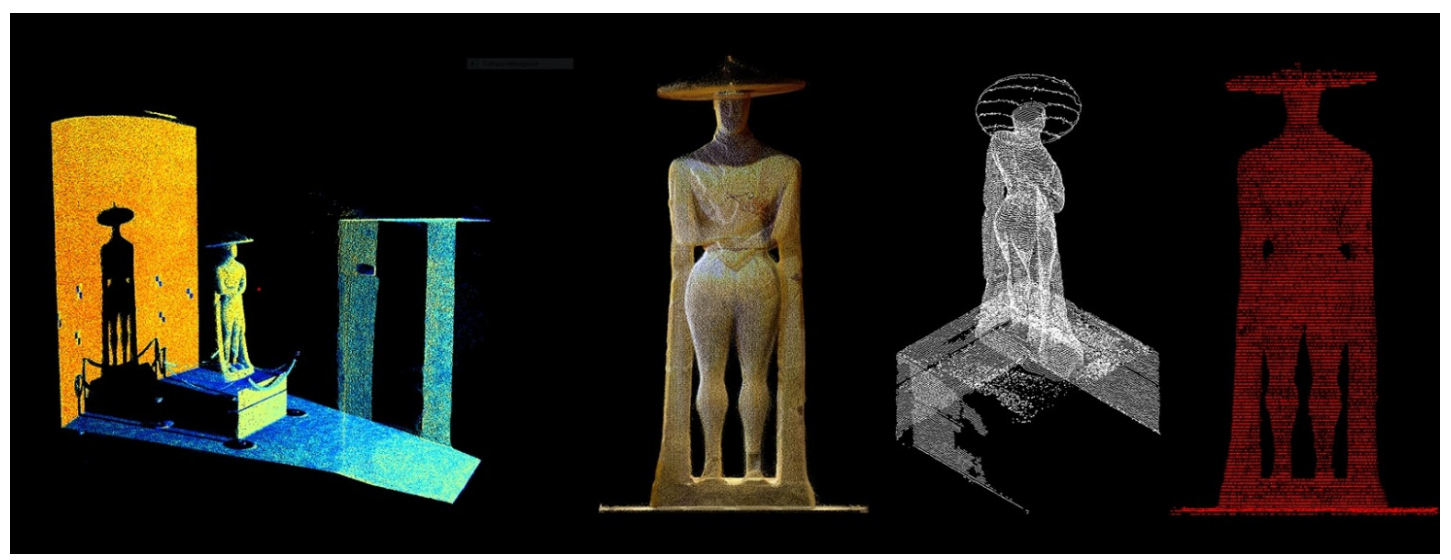

Fig. 37 Esempi di output da laser scanner: viste assonometriche, orto-foto, sezioni vettoriali.

mano tutto ciò che si vuole mettere a confronto. L'archeografia da sola non serve, ma neanche l'archeologia da sola, senza cioè il suo braccio destro, ma sempre più spesso, come si è visto, il "cervello" archeologico non può fare a meno neanche del suo braccio sinistro, che è l'archeometria". ${ }^{112}$

\footnotetext{
111 Architetto, Responsabile tecnico del Laser Scanner per il CAAM.

112 MANNONI 2000, p. 219.
} 
L'approccio utilizzato per le indagini tridimensionali è volto, in questo senso, a una stretta collaborazione e integrazione tra le differenti discipline, in modo da instaurare un dialogo continuo che non si limiti a un semplice confronto e completamento dei dati, ma divenga parte attiva e critica. Il Laser scanner utilizzato per le indagini tridimensionali nel caso del progetto $A R S$, grazie alle attrezzature messe a disposizione dal progetto EuroTeCH, è il GLS 1000 della Topcon: questo tipo di laser scanner si differenzia dalla gran parte dei prodotti simili in commercio, per le caratteristiche versatili che permettono di eseguire rilievi in molteplici contesti di ricerca.

\begin{tabular}{|c|c|}
\hline \multicolumn{2}{|l|}{ PRESTAZIONI DEL SISTEMA } \\
\hline $\begin{array}{l}\text { Portata massima con riflettività } \\
\text { specificata } \\
\text { Portata calcolata al } 18 \% \text { di riflettività }\end{array}$ & $\begin{array}{l}330 \mathrm{~m} \text { al } 90 \%, 150 \mathrm{~m} \text { al } 18 \% \\
150 \mathrm{~m}\end{array}$ \\
\hline $\begin{array}{l}\text { Accuratezza del punto singolo } \\
\text { Distanza } \\
\text { Angolo } \\
\text { Accuratezza di riconoscimento del } \\
\text { target }\end{array}$ & $\begin{array}{l}4 \mathrm{~mm} \text { a } 150 \mathrm{~m} \\
\text { 6" (Verticale) / 6" (Orizzontale) } \\
\text { 3" a } 50 \mathrm{~m}\end{array}$ \\
\hline \multicolumn{2}{|l|}{ SISTEMA DI SCANSIONE LASER } \\
\hline Tipo & A impulsi invisibile agli occhi di classe 1 \\
\hline Velocità di scansione & $3.000 \mathrm{punti} / \mathrm{secondo}$ \\
\hline $\begin{array}{l}\text { Risoluzione } \\
\text { Dimensioni dello spot laser } \\
\text { Distanza tra i punti }\end{array}$ & $\begin{array}{l}6 \mathrm{~mm} \text { a } 40 \mathrm{~m} \\
1 \mathrm{~mm} \text { a } 100 \mathrm{~m}\end{array}$ \\
\hline Immagini digitali a colori & Fotocamera digitale 2.0 Mega pixel \\
\hline
\end{tabular}

Nella tabella le principali caratteristiche tecniche del laser scanner utilizzato

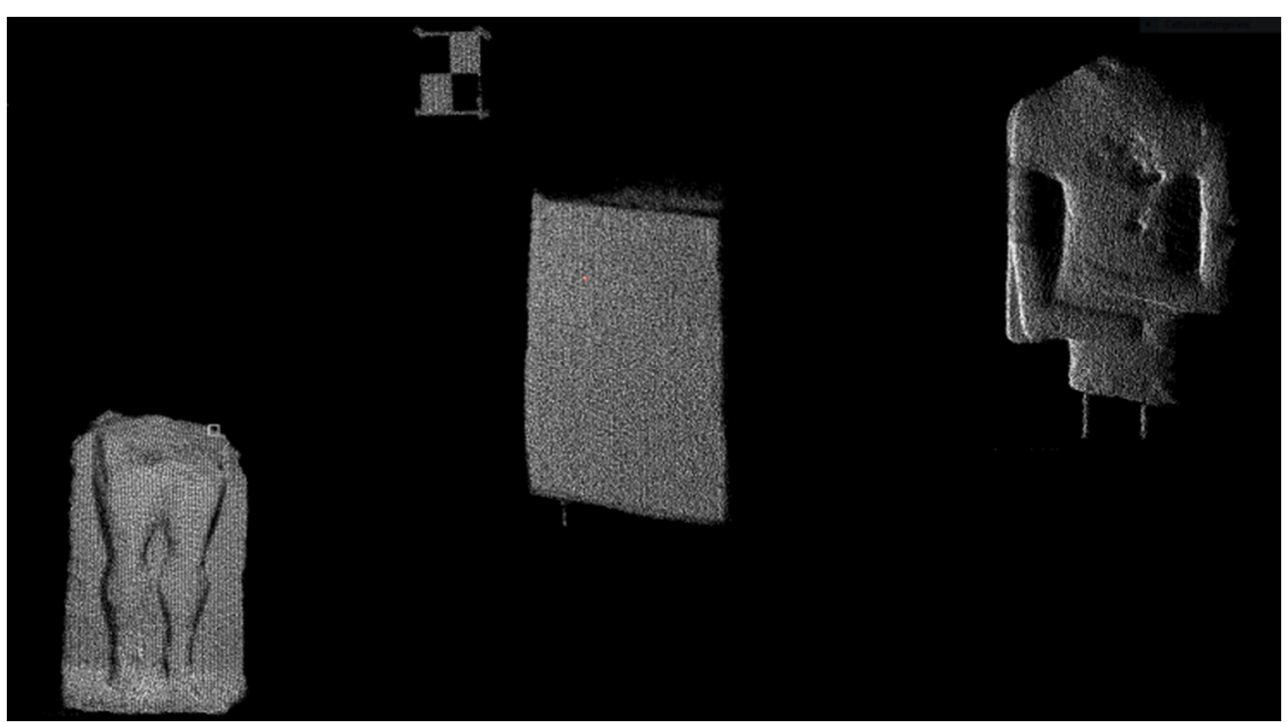

Fig. 38 Intensità di ritorno del raggio laser,con sfumature che vanno dal bianco al nero: - superfici più scure hanno inferiore intensità di ritorno

- superfici grigie hanno una intensità di ritorno media

- superfici bianche alta intensità di ritorno

Le particolari caratteristiche che contraddistinguono questo tipo di strumento, rendono possibile una precisione di $4 \mathrm{~mm}$ uniformi a una distanza di $150 \mathrm{~m}$ e una portata 
massima di scansione di $330 \mathrm{~m}$. La particolarità di poter modulare la distanza di emissione del laser, tramite l'utilizzo di un "raggio sonda", colloca il GLS 100 tra gli strumenti ibridi long range e medium range, con caratteristiche che lo rendono plurifunzionale. Il principio meccanico che il GLS 1000 utilizza per le misure tridimensionali, è paragonabile allo schema dell'intersezione in avanti utilizzato in topografia, essendo nota la distanza tra emettitore e ricevitore del segnale laser e gli angoli di direzione, è possibile calcolare le coordinate xyz del singolo punto.

Le indagini tridimensionali sono ovviamente state effettuate senza alcun contatto con le opere esposte nel museo, adottando particolari accorgimenti che rendono i risultati delle numerose scansioni elaborate, paragonabili tra loro e con altre tecniche di rilievo, come:

- L'utilizzo di un numero minimo di 4 target piani, in vista e alla portata dello strumento identificabili da tutte le scansioni.

- L'utilizzo di software per il riconoscimento automatico dei target in modo da aumentare la precisione di registrazione

- La modulazione della distanza tra emettitore e superficie rilevata e l'angolo di incidenza tra le stesse, per rendere le nuvole di punti omogenee, evitando il più possibile la formazione di rumore

- Parametri costanti di risoluzione HV

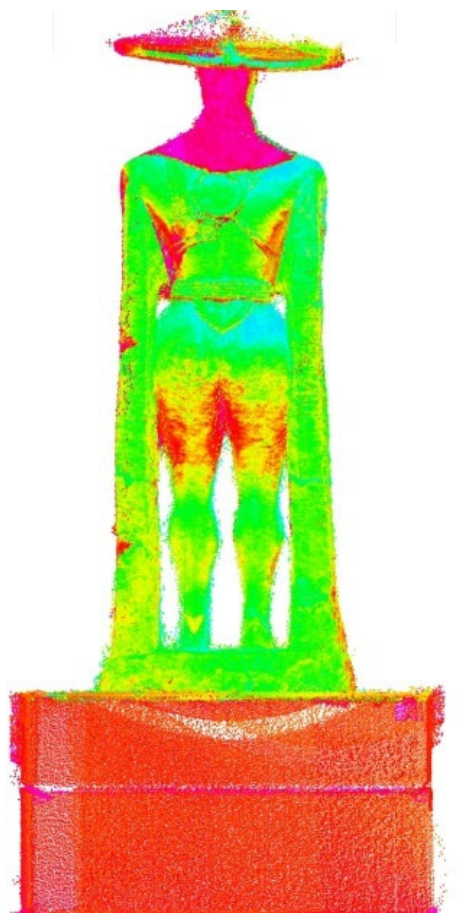

Fig. 39 Nuvola di punti colorata in base a un piano virtuale verticale.
Fattore decisamente rilevante durante le acquisizioni tridimensionali, è l'elevata rapidità di rilievo e il conseguente contenimento dei costi, rimandando la fase di elaborazione dei dati in ufficio. Il post processing, invece, segue un trend decisamente opposto alla fase di rilievo. Nelle tradizionali procedure di rilievo topografico, l'operatore già in fase di acquisizione deve adoperarsi per effettuare una discretizzazione dei punti da misurare, con il laser scanner questo non avviene essendo indifferente alle superfici. La normale conseguenza di questo virtuosismo è l'aumento d'impegno in ufficio, dove il lavoro di pulizia ed elaborazione di enormi quantità di dati incrementano i tempi di gestione della point cloud, rispetto a quelli di acquisizione, anche di 10 volte. Con l'aumento dell'innovazione tecnologica, si vedono incrementi di calcolo anche nei software, in grado ormai di riuscire estrapolare dati, sia per la fruizione da parte dei tecnici, sia per la divulgazione turistica.

Tra gli innumerevoli output che oggi giorno è possibile elaborare, (come le ormai canoniche piante prospetti e sezioni, tour virtuali, orto-prospetti, modellazioni solide, VR, piani quotati, ecc..) particolare attenzione è stata dedicata allo studio dei valori di riflettanza che si riferiscono alla quantità di ritorno dell'impulso laser che impatta sulla superficie, valori generalmente compresi tra 0 e 255 . 
Frankfurter elektronische Rundschau zur Altertumskunde 40 (2020)

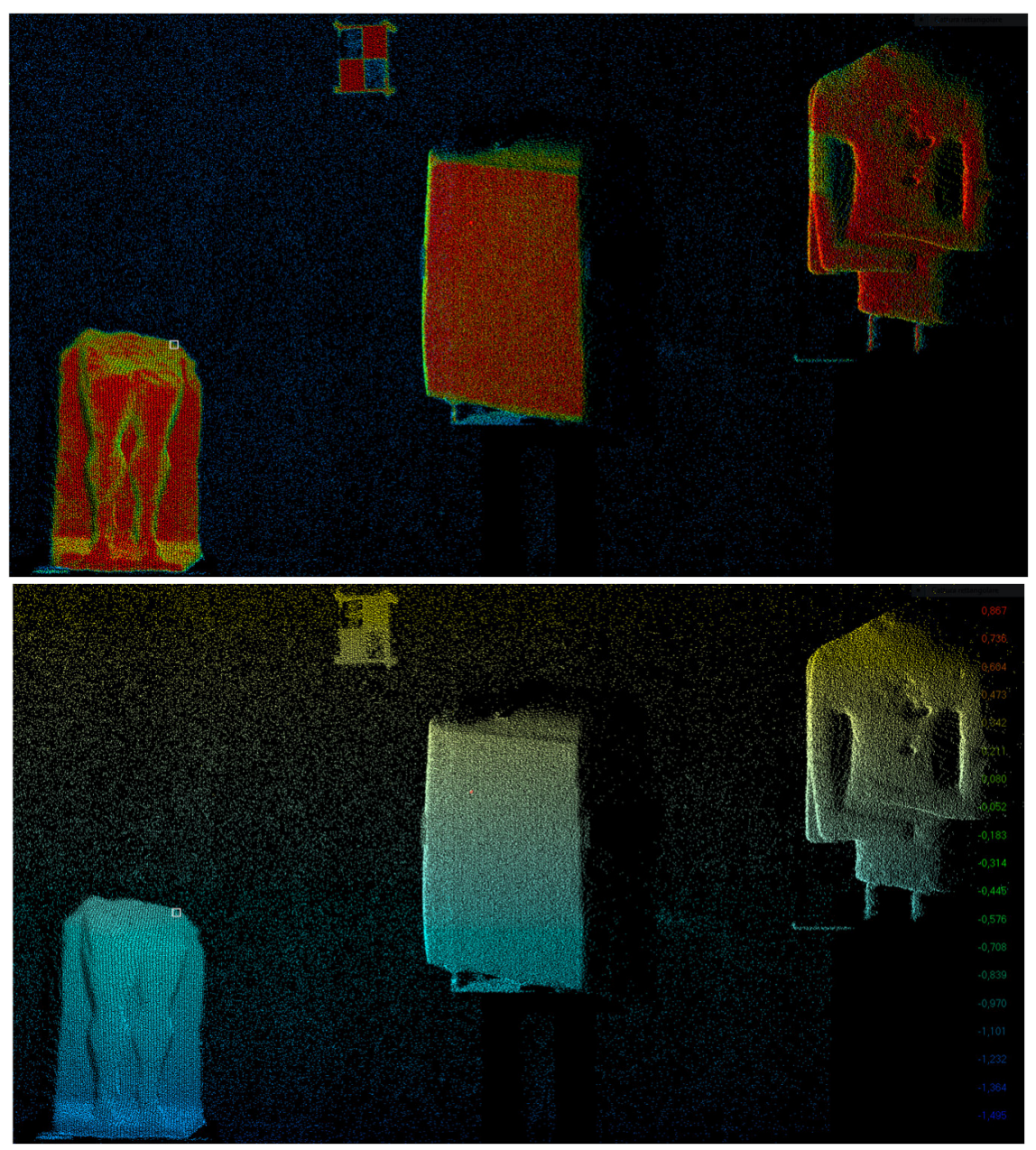

Figg. 40 e 41 Intensità di ritorno del raggio laser, con sfumature che vanno dal rosso al verde al blu :

- superfici più scure con intensità di ritorno inferiore sono colorate di blu

- superfici verdi hanno una intensità di ritorno media

- superfici rosse alta intensità di ritorno

\section{Conclusioni e prospettive}

\section{Rocco d'Errico}

Il De Felice ${ }^{113}$ già dieci anni fa segnalava quanto fosse difficile introdurre le applicazioni di tecniche e metodi digitali ai vari settori dell'archeologia e che ogni tentativo di proporre processi innovativi si è scontrato sempre con l'impermeabilità di un mondo in cui le 'equipe di archeologi sembrano prediligere soluzioni artigianali, votate al pragmatismo nella consuetudine del lavoro quotidiano'.

Oggi la situazione sembra solo apparentemente cambiata perché l'ormai obbligatorio processo di digitalizzazione è solo in parte compiuto, infatti le moderne

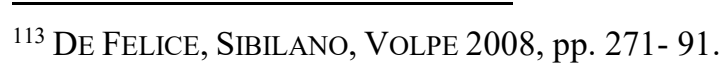


tecniche digitali si utilizzano ancora in maniera poco produttiva senza ricorrere all'acquisizione di competenze specifiche.

La superficialità di questo approccio porta spesso l'archeologo ad arrangiarsi di volta in volta acquisendo e sperimentando nuove tecniche senza però averne il pieno controllo; oppure al contrario a servirsi di tecnici specializzati di cui però difficilmente può controllare le tecniche e i protocolli di analisi.

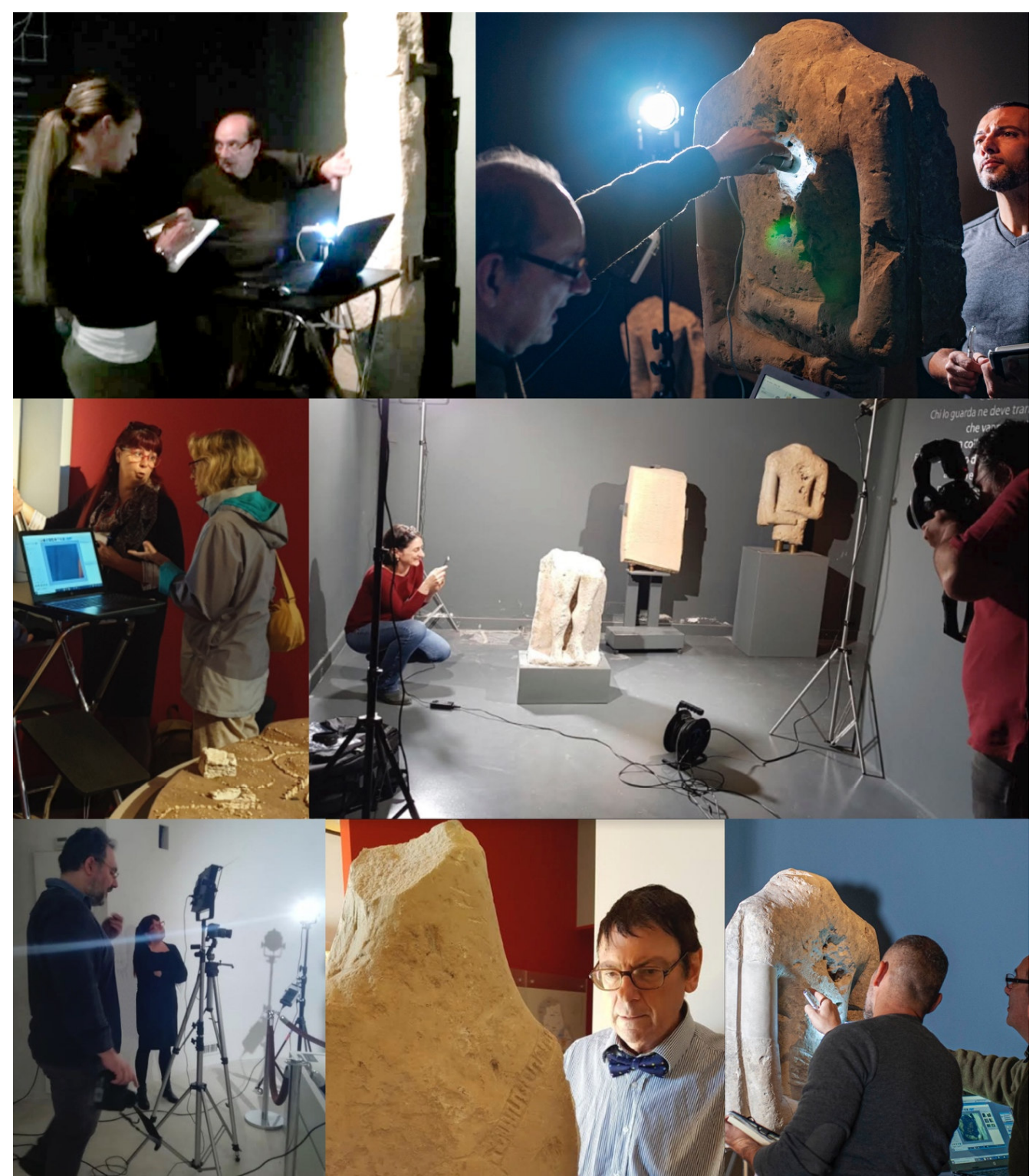

Fig. 42 Alcuni membri dell'equipe a lavoro durante le fasi di raccolta dati sulle sculture conservate nel Museo Archeologico Nazionale di Chieti

Può quindi capitare d'imbattersi in equipe archeologiche in cui l'interazione sia solo apparente e diversi specialisti guardano solo al proprio specifico campo senza sapersi interfacciare con gli altri.

Il protocollo che si propone con il progetto ARS vuole provare a superare tale limitazione sfruttando le competenze specifiche dei vari membri, assegnando ad ognuno il giusto compito ma contemporaneamente condividendo subito dati e opinioni in frequenti riunioni operative. La continua collaborazione e il continuo contatto tra $i$ 
membri dell'equipe, oltre a rendere più facile l'analisi dei dati e la ricostruzione di un quadro storico, rappresenta un'occasione di crescita professionale perché consente ad ognuno di acquisire nuove competenze e di comprendere più a fondo il lavoro degli altri. La condivisione dei dati agevolata dalla stessa natura digitale della ricerca e la possibilità di scambiarsi opinioni facilitata dalle moderne tecnologie di comunicazione porta spesso a scoperte frutto di un vero e proprio lavoro di gruppo.

Un simile protocollo di studio è analisi, risulta estremamente funzionale anche per la valorizzazione turistica delle sculture e si confà al principio che la valorizzazione non sia più solo promozione ma soprattutto costruzione di contenuti e servizi che vengono incontro alle esigenze dei visitatori.

Dalle analisi dei questionari compilati dai visitatori di alcuni musei italiani si ${ }^{114}$ capisce come l'utente cerchi nel museo un'esperienza, vuole riportare a casa un ricordo personale di archeologia esperienziale e quindi avere la possibilità di scegliere cosa vedere, cosa imparare, quali argomenti approfondire. Il cosiddetto turista esperienziale ${ }^{115}$ vuole avere la possibilità di personalizzare la propria visita e quindi deve avere a disposizione qualcosa in più di una guida turistica o di semplici pannelli esplicativi. L'avere a disposizione nuovi dati di tipo analitico, può favorire la possibilità di creare delle ricostruzioni di modelli storici più complete che aiutino nello storytelling e nel creare contenuti multilivello adeguati a varie tipologie di utenti. Inoltre i modelli $3 \mathrm{D}$, i virtual tour, la fotografia ad alta risoluzione possono rendere i contenuti prodotti più accattivanti, interessanti e facilmente accessibili a un vasto e variegato pubblico e portare quindi a compimento il lavoro.

Questo protocollo di studio interdisciplinare quindi aiuta non solo nel portare avanti una ricerca multidisciplinare completa, ma anche nella valorizzazione turistica più sostenibile ed immediata delle sculture: sia perché consente di ricostruire un quadro storico più dettagliato e 'immersivo' per il visitatore, sia perché fornisce i mezzi per una disseminazione del dato archeologico più adeguabile in base alle necessità di diverse fasce di pubblico.

\section{Bibliografia}

Adinolfi G., Carmagnola R., Cataldi M., Marras L., Palleschi V. 2019, 'Recovery of a lost wall painting at the Etruscan tomb of Blue Demons in Tarquinia (Viterbo, Italy) by multispectral reflectometry and UV fluorescence imaging', in Archaeometry, 61, 2, pp. 450-458

Agostini S. 2005. 'Il Guerriero di Capestrano (Museo Archeologico Nazionale d'Abruzzo-Chieti): studi di diagnostica per la conoscenza e la conservazione', in Archeologia nel Mediterraneo, i percorsi d'Italia dal passato al futuro. Paestum VIII Borsa mediterranea del Turismo Archeologico. MIBAC Dipartimento per la Ricerca, l'Innovazione e l'Organizzazione.

AGOSTINI S. 2010. 'Il Guerriero di Capestrano: una scheda archeometrica', in FrANCHI DELL'ORTO 2010, pp. 226-229.

ARMIT I, GRANT PH. 2008. 'Gesture politics and the art of ambiguity: the Iron Age statue from Hirschlanden', Antiquity 82, pp. 409-422.

\footnotetext{
${ }^{114}$ E. BONACINI 2014, pp. 67-78.

${ }^{115}$ L. SOLIMA, 2012.
} 
BonACINI E. 2014. Dal Web alla App. Fruizione e valorizzazione digitale attraverso le nuove tecnologie e $i$ social media, Catania.

BOnenfant P. 1998, Guillaumet J-P., BOYER F.. La statuaire anthropomorphe du premier âge du Fer. Vol. 43. Presses Univ. Franche-Comté, 1998.

BRENDEL O. 1935. ‘Archäologische Funde in Italien', $A A$ 1935, pp. 572-581.

BRINKMANN V.2004. La ricerca della policromia della scultura antica in A.A. V.V., I colori del bianco. Policromia nella scultura antica. Roma.

BRIZIO E. 1902, 'Atri-Necropoli preromana scoperta nel fondo detto la Pretara', NSC.

CALDERINi A., Neri S., RugGeri M. 2007. 'L'iscrizione sul guerriero di Capestrano (ST Ap AQ 2)', in RUGGERI 2007, pp. 46-47.

CELla E. 2012.'La necropoli di Capestrano: nuove acquisizioni', in Mediterranea IX, pp. $57-109$.

CELLINI P. 1992. Falsi e restauri: oltre l'apparenza, Roma.

Ceccarelli A., Fratianni G. 2017. 'Molise', in Archeologia delle Regioni d'Italia, Roma.

CIAnfarani V. 1959 (2008). 'Il guerriero di Capestrano', in G. AloÉ (a cura di), Terra italica e altre storie, Pescara 2008, pp. 34-36.

CIANFARANI V. 1966. 'Stele d'arte medio-adriatica da Guardiagrele', in $B d A$ 1966, pp. $1-6$.

Cianfarani V. 1968. 'Note sul restauro del Guerriero di Capestrano', in Rivista dell'istituto nazionale d'archeologia e storia dell'arte 15, pp. 5-19.

CiAnfarani V. (a cura di) 1969. Antiche civiltà d'Abruzzo, catalogo della mostra (Roma 1969), Roma.

Cianfarani V. 1970. Culture adriatiche d'Italia. Antichità tra Piceno e Sannio prima dei romani, Roma.

CIANFARANI V. 1975. 'Convergenze e divergenze di culture abruzzesi nell'età del ferro', in Introduzione alle antichità adriatiche, atti del I convegno di studi sulle antichità adriatiche (Chieti, Francavilla al Mare, 27-30 giugno 1971), Chieti, pp. 41-51.

CIANFARANI V. 1976. 'Culture arcaiche dell'Italia medio-adriatica', in V. CIANFARANI, D. Lollini, M. ZuFFA (a cura di), PCIA V. Culture arcaiche in Italia medio-adriatica. Civiltà Picena, Roma, pp.1-106.

Cianfarani V., Franchi Dell'Orto L., La Regina A. 1978. Culture adriatiche antiche d'Abruzzo e di Molise, Roma.

Colonna G. 1955. 'Pallanum. Una città dei Frentani', in $\mathrm{ArchCl}$, 1955, pp. 164-178.

ColonNA G. 1992. 'Apporti etruschi all'orientalizzante «piceno»: il caso della statuaria', in La civiltà picena nelle Marche. Studi in onore di Giovanni Annibaldi, atti del convegno (Ancona 1988), Ripatransone, 1992, pp. 92-127.

Colonna G. 1999. 'La scultura in pietra', in Franchi Dell'Orto L. (a cura di), Piceni Popolo d'Europa, Roma, pp. 104-109. 
Colonna G. 2001.'La scultura in pietra', in Eroi e Regine. Piceni Popolo d'Europa, Roma.

Colonna G., Peroni R. 1961, s.v. 'Italica arte', in EAA IV, pp. 251-274 (reperibile online al link: http:/www.treccani.it/enciclopedia/arte-italica_\%28Enciclopediadell $\% 27$-Arte-Antica $\% 29 /$ )

CraWford M.H. (ed.) 2011. Imagines Italicae. A corpus of Italic inscriptions, vols. 13, London.

CUmONT F. 1935. 'Un kouros italique découvert à Capestrano (Abruzzes)', in CRAI, 79, pp. 39-40.

Despini A., SchÜRMANN W. AND GiSler J-R. 2009. 'Gold Funerary Masks', Antike Kunst 52. Jahrg., pp. 20-65

De Felice G., Sibilano M.G., Volpe G. 2008. 'Ripensare la documentazione archeologica: nuovi percorsi per la ricerca e la comunicazione', in Archeologia e Calcolatori, n19, Firenze.

D'ERCOLE, V. 1991. 'La necropoli di Scurcola Marsicana', Il fucino e le aree limitrofe nell'antichità, Roma 1991, pp. 252-270.

D'ERCole V. 2007.'La statuaria in pietra nell'Abruzzo costiero settentrionale', in Ruggeri M. (a cura di), Guerrieri e Re dell'Abruzzo antico, Pescara pp. 75-79.

D’ERCole V. 2010. 'Le armi e gli armati'. in Chiaramonte Trerè C., D’Ercole V., Scoтti C. (a cura di), La necropoli di Campovalano. Tombe orientalizzanti e arcaiche II, Oxford.

D'ERCole V. 2014. 'Menhir, stele e statue in pietra in area medio-adriatica', in G. Baldelli e F. Lo Schiavo (a cura di) Amore per l'Antico. Dal Tirreno all'Adriatico, dalla Preistoria al Medioevo e oltre. Studi di Antichità in ricordo di Giuliano De Marinis, Roma pp. 463-474.

D'ERCOLE V. 2014 B. 'Per una definizione della Koinè culturale vestina', in BourdiN S. E D'ERCOLE V. (a cura di), I Vestini e il loro territorio, dalla Preistoria al Medioevo, Roma pp. 29-61.

D'ERCole V. 2015. 'Capestrano: 10.000 anni di storia prima di Roma', in CHIARIZIA G. E IAgnemma L. (a cura di), Capestrano nella Valle Tritana, L’Aquila.

D’ERCole V. 2015 B.'Le spade tipo Capestrano: tipologia, distribuzione, cronologia, funzione e significato', in GiLOtTA F. E TAGLIAMONTE G. (a cura di), Sui due versanti dell'Appennino. Necropoli e distretti culturali tra VII e VI sec. a. C., Roma pp. 245268.

D'ERCOLE V. 2017. 'Scultura funeraria dal mondo medio-adriatico: statue, stele, cippi', in Steingraber S. (a cura di), Cippi, Stele, Statue-Stele e Semata. Testimonianze in Etruria, nel mondo italico e in Magna Grecia dalla prima Età del ferro fino all'Ellenismo, Pisa.

D'Ercole V., Acconcia V., Cesana D. 2018. La necropoli di Capestrano, I. Scavi d'Ercole 2003-2009, Oxford.

D'Ercole V., Acconcia V., Cesana D., Menozzi O. in c. s, La necropoli di Capestrano, II. Scavi antichi (1934-1992) e recenti (2010-2012), in corso di stampa. 
D'ERCOLE V., MARTELLONE A. 2005.'La problematica dei confini nella protostoria d'Abruzzo', in Atti Convegno Deputazione di Storia Patria Il confine nel tempo, L'Aquila.

D'Ercole V., Martellone A. 2006.'Il santuario di Monte Giove a Penna Sant'Andrea', in Museo Civico Archeologico 'F. Savini' Teramo, Teramo.

D’Ercole, V., PAPI R., Grossi G. 1990, Antica terra d'Abruzzo, L’Aquila.

Donohue A. A. 1987, Xoana and the Origins of Greek Sculpture, ACS, 15, 1987

DurAnTe, M. 1978. 'I dialetti medio-italici', PCIA, VI (Roma), pp. 789-824.

EGG M. 1996. Das hallstattzeitliche Fürstengrab von Strettweg bei Judenburg in der Obersteiermark, Mainz am Rhein.

EGG M. 2004. 'Die Wiederentdeckung eines ost-hallstättischen Fürstengrabes. Anmerkungen zum Fürstengrab im Hartnermichelkogel 1 bei Kleinklein (Gem. Grossklein, Bez. Leibniz) in der Weststeiermark', in JbRGZM, 51, 2001, pp. 93-126.

Esposito A.M., MAGgiani A. 2006, 'Casale Marittimo: lo scavo e la mostra', in voN ELES P. (a cura di), La ritualità funeraria tra età del ferro e orientalizzante in Italia, atti del convegno (Verucchio, 26-27 giugno 2002), Pisa-Roma 2006, pp. 197-202.

FERRI S. 1949. 'Osservazioni intorno al guerriero di Capestrano', in BdA 1949, pp. 1-9.

FRANCHI DELL'ORTO L. 2010. 'Il guerriero di Capestrano e la statuaria medio-adriatica', in FrANCHI DELL'ORTO, LA REgINA, BUONOCORE 2010, pp. 180-225.

Franchi dell'Orto L. (a cura di) 2010. Pinna Vestinorum e il Popolo dei Vestini, Roma.

Gamba M., GambacurTa G. 2011. 'Le statue di Gazzo Veronese al confine fra Veneti ed Etruschi' in Tra Protostoria e Storia. Studi in onore di Loredana Capuis, Roma.

Govi E. 2014. 'Lo studio delle stele felsinee. Approccio metodologico e analisi del linguaggio figurativo', in AnnFaina 21, pp. 127-186.

GRAELLS I FABREGATR. 2018.'Le corazze nei santuari dell'Italia meridionale', in Graells R. E LongoF. (a cura di) Armi votive in Magna Grecia, RGZM, Mainz.

GuIDOBALDI M.P. 1995. La romanizzazione dell'ager Praetutianus (secoli III-I a. C.), Perugia.

IACULLI G. 1982. 'Attività della Soprintendenza Archeologica d'Abruzzo 1970-1979', in Quaderni dell'Istituto di Archeologia di Chieti, 2, pp. 194-216.

IANNACCONE R. 2014. Tecniche di Imaging Innovative per la Messa a Punto di un Protocollo Integrato per la Caratterizzazione dei Pigmenti Utilizzati nell'Antichità, tesi di dottorato, Università degli Studi di Firenze, a.a. 2012-2014.

LETTA C. 1979. 'Il territorio del Fucino in età preromana e romana', in SERVIDIO A., Radmilli A.M., Letta C., Messineo G. et AL. (a cura di), Fucino cento anni, Avezzano, 1979, pp. 99-138.

LA Regina A. 1986. 'Le stele paleosabelliche', in Documenti dell'Abruzzo Teramano, La Valle del Medio e Basso Vomano, vol. II, Roma, pp. 125-130. 
LA Regina A. 2010. 'Il guerriero di Capestrano e le iscrizioni paleosabelliche', in L. FRANCHI DELl'Orto (a cura di), Pinna Vestinorum e il popolo dei Vestini, Roma pp. 230-273.

Maggiani A. 1999. 'Le statue di Casale Marittimo', in Esposito A.M. (a cura di), Principi guerrieri: la necropoli etrusca di Casale Marittimo, catalogo della mostra (Cecina 1999), Milano 1999, pp. 33-39.

Maggiani A. 2015. 'Dentro il recinto: l'arredo scultoreo delle tombe a tumulo di Vetulonia', in La delimitazione dello spazio funerario in Italia dalla Protostoria all'età Arcaica: recinti, circoli, tumuli. Annali della Fondazione per il Museo Claudio Faina, volume XXII, Roma pp. 197-221.

MALNATI L. 2002. 'Monumenti e stele in pietra preromani in Veneto', in AKEO. I tempi della scrittura. Veneti antichi. Alfabeti e documenti, catalogo della mostra (Montebelluna 2001-2002), Cornuda 2002, pp. 127-138.

Magiangeli G. 1978. Il guerriero italico di Capestrano, Spoleto.

MARCHESI M. 2011. Le sculture di età orientalizzante in Etruria padana, Bologna.

Marinetti A. 1984. Popoli e civiltà dell'Italia antica. VI. Lingue e dialetti: Aggiornamenti ed indici Roma.

MARInetTi A. 1985. A., Le iscrizioni Sud-Picene. Testi, Firenze.

MARzOLI, D. 2003. 'Eigenheiten der ältesten Grossplastik Mittleuropas: die Statue von Hirschlanden', Madrider Mitteilungen 44, pp. 196-214.

Menozzi O. 2019. 'Themis, dike e dikaiosyne: iconografia, culti e santuari nel mondo greco', in Bultrighini U. E Di Mauro E. (a cura di), Koinos Logos. Pensare Giustizia tra Antico e Contemporaneo, Chieti, pp. 293-315.

Menozzi O., TAMBURrino C. 2012.'Laser Scanning and Infra-Red Thermographic Prospecting for Diagnostic Mapping and Restoration Projects: The Case the Painted Tombs at Cyrene (Libya)', International Journal Of Heritage In The Digital Era, vol. I, pp. 343-349.

Meschini A., Pelliccio A. 2013, 'Il colore nel rilievo strumentale: laser scanner, termografia e postprocessing dei dati in un sistema GIS', in RosSi M., SINISCALCO A. (a cura di), Colore e Colorimetria. Contributi multidisciplinari, Salerno, pp.70-81.

MoretTi G. 1936. Il guerriero italico di Capestrano, (M.A.L.) Roma.

MoretTI G. 1936-37. Il guerriero italico e la necropoli di Capestrano, in BPI n.s. I, 1936-37, pp. 94-112.

PAPI R. 1981. 'Un frammento inedito di scultura italica in Abruzzo', in Quaderni dell'Istituto di Archeologia di Chieti, 2, pp.16-20, note 4 e 7.

ONORATO G. O. 1960, La ricerca archeologica in Irpinia, Avellino 1960

Prosdocimi A.L. 1977, 'Il lessico istituzionale italico: tra linguistica e storia', La Cultura Italica (Pisa), pp.29-74.

Prosdocimi A.L. 1978, 'Le lingue Italiche', PCIA, VI, Roma.

Prosdocimi A.L. 1985, 'I Safini delle iscrizioni sudpicene', Proceedings of the Cambridge Philological Society, Cambridge, pp. 35-56. 
Prosdocimi A.L. 1987, 'Sabinità e (pan)italicità linguistica', DialA 5, pp.1-13.

Rainini I. 1976, Bottini A., Isnenghi Colazzo S., Valle d'Ansanto. Rocca S. Felice (Avellino). Il deposito votivo del santuario di Mefite, «NSA» s. VIII, 30, 1976, pp. 359524.

RugGeri M. (a cura di) 2007. Guerrieri e re dell'Abruzzo antico, Pescara.

Tomedi G. 2000.Italische Panzerplatten und Panzerscheiben, P. B. F. III, 3, Stuttgart.

Rossi M., Siniscalco A. 2013. Colore e Colorimetria. Contributi multidisciplinari, Salerno

Salerno, E., Tonazzini, A., Grifoni, E., Lorenzetti, G., Legnaioli, S., Lezzerini, M., PALlesCHI, V. 2014, 'Analysis of Multispectral Images in Cultural Heritage and Archaeology', in JALS (Journal of Applied and Laser Spectroscopy), 1, pp. 22-27.

SoliMA L .2012. Il museo in ascolto. Nuove strategie di comunicazione per i musei statali, Catanzaro.

SCHICKLER H. 1999, 'Il guerriero di Hirschlanden', in FrANCHI DELL'ORTO L. (a cura di), Piceni Popolo d'Europa, Roma, pp. 23-29.

Tonazzini A., Gerace I., Martinelli F. 2010, 'Multichannel blind separation and deconvolution of images for document analysis', in IEEE Transactions on Image Processing, 19 (4), pp. 912-925.

UsAi U. 1993. 'Capestrano', Notiziario Scavi e Scoperte Rivista di Scienze Preistoriche, Firenze.

VERRI G. 2009. The application of visible-induced luminesacence imaging to the examination of museum objects. Proceeding SPIE 7391, O3A: Optics for Arts, Architecture and Acheology II, 739105.

WeIDig J. 2014. Bazzano - Ein Gräberfeld bei l'Aquila (Abruzzen). Die Bestattungen des 8.-5. Jahrhunderts v. Chr., Mainz.

WeIDIGJ. 2016. 'I draghi appenninici. Appunti sulle raffigurazioni degli animali fantastici italici tra Abruzzo, Umbria e Marche', in Aristonotos Scritti per il Mediterraneo antico, Nuovi studi sul bestiario fantastico di età orientalizzante nella penisola italiana.Quaderni, n. 5, Trento pp. 247-272.

Williams G, Williams R. 2005.Ultraviolet, Infrared\& Fluorescence Photography, 2005.

ZÜRN H. 1964. 'An anthropomorphic Hallstatt stele from Germany', Antiquity 38, pp. 224-6.

ZÜRN H. 1970, Hallstattforschungen in Nordwürttemberg. Die Grabhügel von Asperg (Kr. Ludwigsburg), Hirschlanden (Kr. Leonberg), und Mühlacker (Kr. Vahingen), Stuttgart.

\section{Contatti}

Oliva Menozzi (Universitá G.d'Annunzio di Chieti-Pescara)

E-Mail: $\underline{\text { o.menozzi@unich.it }}$ 